\title{
O uso do estimador residual no refinamento adaptativo de malhas em elementos finitos
}

Marco Alexandre Claudino

\author{
DISSERTAÇÃO APRESENTADA \\ $\mathrm{AO}$ \\ Instituto DE MatemáticA E EstatísticA \\ DA \\ Universidade De SÃo Paulo \\ PARA \\ OBTENÇÃO DO TÍTULO \\ DE \\ Mestre em CiÊnCIAS \\ Programa: Matemática Aplicada \\ Orientador: Prof. Dr. Nelson Mugayar Kuhl
}

Durante o desenvolvimento deste trabalho o autor recebeu auxílio financeiro do $\mathrm{CNPq}$

São Paulo, Março de 2015 


\section{O uso do estimador residual no refinamento adaptativo de malhas em elementos finitos}

Esta versão da dissertação contém as correções e alterações sugeridas pela Comissão Julgadora durante a defesa da versão original do trabalho, realizada em 26/03/2015. Uma cópia da versão original está disponível no

Instituto de Matemática e Estatística da Universidade de São Paulo.

Comissão Julgadora:

- Prof. Dr. Nelson Mugayar Kuhl (orientador) - IME-USP

- Prof. Dr. Luis Carlos de Castro Santos - IME-USP

- Prof. Dr. Ernani Vitillo Volpe - EP-USP 


\section{Agradecimentos}

Primeiramente, agradeço a Deus pela saúde, pela coragem e pela fé que sempre me guiou frente aos momentos mais complicados da vida.

A minha esposa, Stéfany, pelo carinho, atenção e paciência tidos comigo desde o início. Seu apoio e incentivo nos momentos mais difíceis desta jornada foram fundamentais para que eu chegasse até aqui.

Aos meus pais, Marco e Oneide, e minha irmã, Cristiane, pelo apoio e incentivo constantes.

Ao professor Nelson Kuhl por toda paciência e atenção, além dos valorosos conselhos dados durante as reuniões de iniciação científica que se tornaram um trabalho de conclusão de curso e posteriormente evoluíram para o mestrado que se encerra com a conclusão desta dissertação.

Aos amigos Willian Hans Corrêa (79), Lucas Reis e Eli Enrico pela companhia e apoio durante a jornada acadêmica. Em especial, ao brother Guilherme Salomão pela amizade e companheirismo, além do auxílio nas disciplinas de análise.

Aos professores Alexandre Roma e Pedro Peixoto pelos valorosos conselhos, sugestões e orientações durante o período em que fui estagiário PAE.

Aos professores Luis Carlos de Castro Santos e Ernani Vitillo Volpe por aceitarem participar da comissão avaliadora e pelas valorosas sugestões dadas para a melhoria deste trabalho.

Aos professores Claudio Possani, Pierluigi Benevieri, Ricardo Freire, Salvador Zanata e Saulo Maciel, cujos conhecimentos transmitidos em aula foram muito importantes para o meu desenvolvimento acadêmico e me ajudaram a entender um pouco mais do vasto universo que consiste a matemática (e suas aplicações).

Ao $\mathrm{CNPq}$ pelo suporte financeiro. 
"If something is obviously not impossible Then must be a way to doing it". 


\section{Resumo}

Claudino, M.A. O uso do estimador residual no refinamento adaptativo de malhas em elementos finitos. 2015. 82 f. Dissertação - Instituto de Matemática e Estatística, Universidade de São Paulo, São Paulo, 2015.

Na obtenção de aproximações numéricas para Equações Diferenciais Parciais Elípticas utilizando o Método dos Elementos Finitos (MEF) alguns problemas apresentam valores maiores para o erro somente em algumas determinadas regiões do domínio como, por exemplo, regiões onde existam singularidades na solução contínua do problema. Uma possível alternativa para reduzir o erro cometido nestas regiões é aumentar o número de elementos nos trechos onde o erro cometido foi considerado grande. A questão principal é como identificar essas regiões, dado que a solução do problema contínuo é desconhecida.

Neste trabalho iremos apresentar a chamada estimativa residual, que fornece um estimador do erro cometido na aproximação utilizando apenas os valores conhecidos dos contornos e a aproximação obtida sobre uma dada partição de elementos. Vamos discutir a relação entre a estimativa residual e o erro cometido na aproximação, além de utilizar as estimativas na construção de um algoritmo adaptativo para as malhas em estudo.

Utilizando o software FreeFem ++ serão obtidas aproximações para a Equação de Poisson e para o sistema de equações associado à Elasticidade Linear e por meio do estimador residual será analisado o erro cometido nas aproximações e a necessidade do refinamento adaptativo das malhas.

Palavras-chave: Método dos Elementos Finitos, Estimativas residuais, Refinamento adaptativo de malhas. 


\section{Abstract}

Claudino, M.A. The use of the residual estimation in adaptive mesh refinement of finite element. 2015. 82 f. Dissertação - Instituto de Matemática e Estatística, Universidade de São Paulo, São Paulo, 2015.

In obtaining numerical approximations for solutions to Elliptic Partial Differential Equations using the Finite Element Method (FEM) one sees that some problems have higher values for the error only in certain domain regions such as, for example, regions where the solution of the continous problem is singular. A possible alternative to reduce the error in these regions is to increase the number of elements in the partions where the error was considered large. The main issue is how to identify these regions, since the solution of the continuous problem is unknown.

In this work we present the so-called residual estimate, which provides an error estimation approach which uses only the known values on the contours and the obtained approximation on a given discretization. We will discuss the relationship between the residual estimate and the error, and how to use the estimate for adaptively refining the mesh.

Solutions for the Poisson equation and the Linear elasticity system of equations, and the residual estimates for the analysis of mesh refinement will be computed using the FreeFem ++ software.

Keywords: Finite Element Method, Residual estimates, adaptive mesh refinement. 


\section{Sumário}

$\begin{array}{ll}\text { Lista de Figuras } & \text { ix }\end{array}$

Lista de Tabelas $\quad$ xi

1 Introdução $\quad 1$

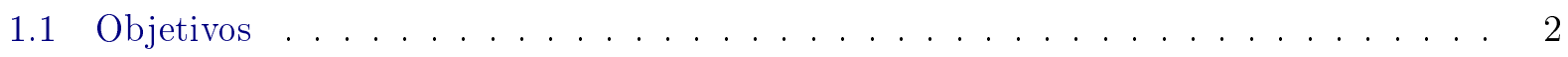

1.2 Organização do Trabalho . . . . . . . . . . . . . . . . . . . 2

2 Conceitos, resultados preliminares e obtenção das aproximações 5

2.1 Ferramentas de análise funcional . . . . . . . . . . . . . . . . . 5

2.2 Formulação Equivalente . . . . . . . . . . . . . . . . . . . 9

2.3 Problemas de valor de contorno . . . . . . . . . . . . . . . . . . 9

2.4 Método de Ritz-Galerkin . . . . . . . . . . . . . . . . . . . . . . 10

2.4 .1 Partições do domínio . . . . . . . . . . . . . . . . . . . . . . . 11

2.4 .2 Funções de forma e funções teste . . . . . . . . . . . . . . . . . . 13

2.4.3 Operador de quasi-interpolação . . . . . . . . . . . . . . . . . . . . . . . 14

2.4.4 Função de salto $(j u m p) \ldots \ldots \ldots \ldots \ldots \ldots$

2.4.5 Construção das aproximações . . . . . . . . . . . . . . . . . . 15

2.4.6 Estimativas na aproximação . . . . . . . . . . . . . . . . 17

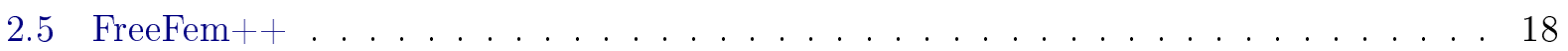

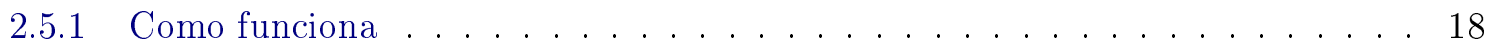

2.5 .2 Outros comandos . . . . . . . . . . . . . . . . . . . . . 19

2.6 Refinamento das malhas de estudo . . . . . . . . . . . . . . . . . . 19

2.6 .1 Particionamento uniforme . . . . . . . . . . . . . . . . . . . . 19

2.6.2 Particionamento orientado por funções indicadoras . . . . . . . . . . . . 20

3 Problema de Poisson $\quad 23$

3.1 Formulação fraca . . . . . . . . . . . . . . . . . . . . . . 23

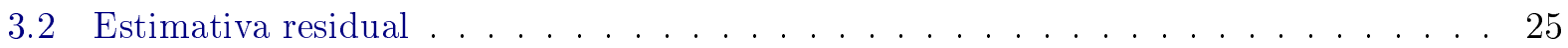

3.3 Simulações Numéricas . . . . . . . . . . . . . . . . . . . . . . . . . 29

3.3.1 Problema com solução conhecida . . . . . . . . . . . . . . . . . 29

3.3.2 Problema com singularidade . . . . . . . . . . . . . . . . 34

4 Elasticidade Linear $\quad 39$

4.1 As equações da elasticidade linear . . . . . . . . . . . . . . . . . . . . . 39

4.2 Formulação variacional (fraca) do problema: Minimizando a energia associada . . . . 40 
4.3 Resultados auxiliares . . . . . . . . . . . . . . . . . . . . 41

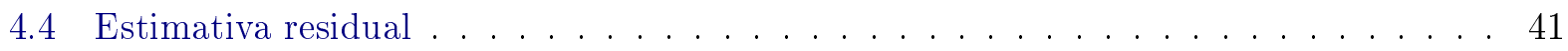

4.5 Simulações Numéricas . . . . . . . . . . . . . . . . . . . . . 43

$\begin{array}{lll}5 & \text { Conclusões } & 47\end{array}$

A Construção do estimador residual para o problema de Elasticidade Linear $\quad 49$

A.1 Construção do estimador . . . . . . . . . . . . . . . . . . . . . . 49

$\begin{array}{ll}\text { B Códigos utilizados } & 51\end{array}$

$\begin{array}{ll}\text { C Figuras } & 63\end{array}$

C.1 Problema de Poisson . . . . . . . . . . . . . . . . . . . 63

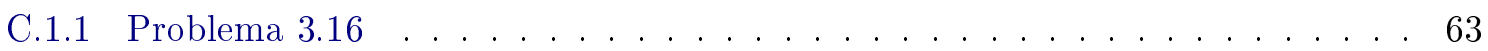

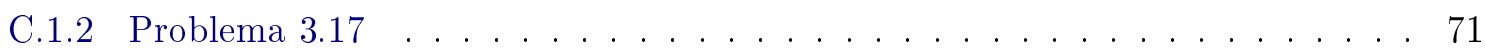

C.2 Elasticidade Linear . . . . . . . . . . . . . . . . . . . . . . 75 


\section{Lista de Figuras}

2.1 Exemplos de malhas de um domínio $\Omega$. A figura a) mostra uma malha admissível enquanto que a figura b) mostra uma malha não admissível, pois possui um elemento cujos vértices estão situados nos pontos médios das arestas de outros elementos. . . . 11

2.2 Exemplos dos conjuntos apresentados na definição $(2.4 .5) \ldots \ldots \ldots$. . . . . . . 12

2.3 As figuras (a) e (b) mostram elementos lineares em $\mathbb{R}^{2}$ e a figura (c) apresenta o elemento linear em $\mathbb{R}^{3} \ldots \ldots \ldots \ldots \ldots \ldots$

2.4 Elemento triangular de três nós . . . . . . . . . . . . . . . . . . . . . . 15

2.5 Montagem da matriz global de rigidez e do vetor global de forças. [22] . . . . . . . 16

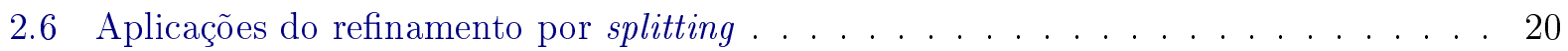

2.7 Malha inicial e estimativa do erro cometido. As cores mais escuras indicam um maior erro cometido nestas regiões. . . . . . . . . . . . . . . . . . . . . . 20

2.8 Malha obtida pelo refinamento indicado pelo estimador de erro . . . . . . . . . . 21

3.1 Solução exata do problema $(3.16) . \ldots \ldots \ldots \ldots \ldots$

3.2 Resultados obtidos sobre os elementos triangulares. . . . . . . . . . . . . . . . . 30

3.3 Resultados obtidos na primeira iteração do refinamento uniforme. . . . . . . . . . . . 31

3.4 Resultados obtidos na primeira iteração do refinamento adaptativo. . . . . . . . . . . 31

3.5 Resultados obtidos na quarta iteração do refinamento uniforme. . . . . . . . . . . . . 32

3.6 Resultados obtidos na quarta iteração do refinamento adaptativo. . . . . . . . . . . . 32

3.7 Comportamento do estimador de erro nas iterações de refinamento adaptativo. . . . 33

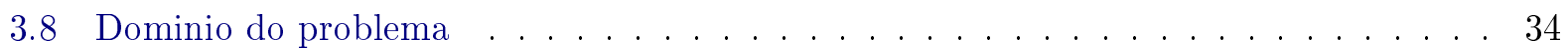

3.9 Resultados obtidos sobre os elementos triangulares no problema $3.17 \ldots \ldots$. . . . . . 35

3.10 Resultados da primeira iteração do refinamento uniforme. . . . . . . . . . . . . . 36

3.11 Resultados da primeira iteração do refinamento adaptativo. . . . . . . . . . . . . 36

3.12 Relação entre o erro cometido e o número de nós da malha. . . . . . . . . . . . . . 37

3.13 Comparativo das aproximações obtidas na quarta iteração de refinamento. . . . . . . 38

4.1 Malha inicial. . . . . . . . . . . . . . . . . . . . . 43

4.2 Resultados obtidos na malha inicial. . . . . . . . . . . . . . . . . . 43

4.3 Deformação sofrida pela chapa (fator de escala $10^{6}$ ). . . . . . . . . . . . . . . 44

4.4 Aproximação obtida na terceira iteração do refinamento uniforme. . . . . . . . . . . . 44

4.5 Aproximação obtida na terceira iteração do refinamento adaptativo. . . . . . . . . . 45

4.6 Relação entre o erro cometido e o número de nós da malha. . . . . . . . . . . . . . 45

4.7 Comparação dos resíduos obtidos na terceira iteração de refinamento. . . . . . . . . . 46 
C.1 Aproximação obtida com elementos lineares - Fig (3.2a) . . . . . . . . . . . . . 63

C.2 Residuo obtido na aproximação - Fig $(3.2 b) \ldots \ldots \ldots \ldots$. . . . . . . 64

C.3 Aproximação obtida na primeira iteração do refinamento uniforme - Fig (3.3a) . . . . 64

C.4 Residuo obtido na aproximação da malha gerada pela primeira iteração do refina-

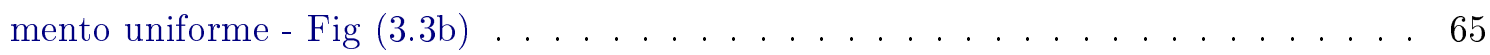

C.5 Aproximação obtida na primeira iteração do refinamento adaptativo - Fig (3.4a) . . . 65

C.6 Residuo obtido na aproximação da malha gerada pela primeira iteração do refinamento adaptativo - Fig $(3.4 b) \ldots \ldots \ldots 66$

C.7 Aproximação obtida na quarta iteração do refinamento uniforme - Fig (3.5a) . . . . . 66

C.8 Residuo obtido na aproximação da malha gerada pela quarta iteração do refinamento

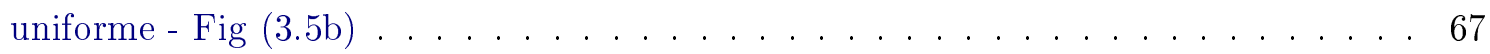

C.9 Aproximação obtida na quarta iteração do refinamento adaptativo - Fig (3.6a) . . . 67

C.10 Residuo obtido na aproximação da malha gerada pela quarta iteração do refinamento adaptativo - Fig $(3.6 b) \ldots \ldots \ldots \ldots \ldots$

C.11 Estimador residual obtido na primeira iteração do refinamento adaptativo - Fig (3.7a) 68

C.12 Estimador residual obtido na segunda iteração do refinamento adaptativo - Fig (3.7b) 69

C.13 Estimador residual obtido na terceira iteração do refinamento adaptativo - Fig (3.7c) 69

C.14 Estimador residual obtido na quarta iteração do refinamento adaptativo - Fig (3.7d) 70

C.15 Aproximação inicial para o problema 3.17 - Fig (3.9a) . . . . . . . . . . . . 71

C.16 Estimador residual obtido para a aproximação inicial - Fig $(3.9 \mathrm{~b}) \ldots \ldots$. . . . . . 71

C.17 Aproximação obtida na primeira iteração do refinamento uniforme - Fig (3.10a) . . . 72

C.18 Estimador residual obtido na primeira iteração do refinamento uniforme - Fig (3.10b) 72

C.19 Aproximação obtida na primeira iteração do refinamento adaptativo - Fig (3.11a) . . 73

C.20 Estimador residual obtido na primeira iteração do refinamento adaptativo - Fig (3.11b) 73

C.21 Aproximação obtida na quarta iteração do refinamento uniforme - Fig (3.12a) . . . . 74

C.22 Estimador residual obtido na quarta iteração do refinamento adaptativo - Fig (3.12b) 74

C.23 Aproximação inicial para o problema da placa com furo - Fig (4.2a) . . . . . . . . 75

C.24 Estimador residual obtido na aproximação inicial - Fig $(4.2 b) \ldots \ldots$. . . . . . 76

C.25 Deslocamento obtido na terceira iteração de refinamento uniforme - Fig (4.4a) . . . 77

C.26 Estimador residual obtido na terceira iteração de refinamento uniforme - Fig (4.6a) . 78

C.27 Estimador residual obtido na terceira iteração de refinamento adaptativo - Fig (4.6b) 79 


\section{Lista de Tabelas}

3.1 Comparação entre o refinamento uniforme e o refinamento adaptativo. . . . . . . . . 32

3.2 Comparação entre o refinamento uniforme e o refinamento adaptativo na quarta iteração de refinamento. . . . . . . . . . . . . . . . . . . . . . 33

3.3 Comparação entre o número de nós de cada um dos refinamentos para obter um erro L2 de mesma ordem. . . . . . . . . . . . . . . . . . . . . . . 34

3.4 Comparação entre o refinamento uniforme e o refinamento adaptativo na primeira iteração de refinamento. . . . . . . . . . . . . . . . . . . . 36

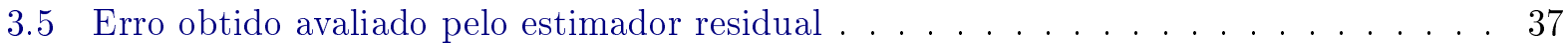

4.1 Erro estimado pelo estimador residual em cada uma das iterações de refinamento. . . 44 


\section{Capítulo 1}

\section{Introdução}

Na simulação numérica de problemas físicos, como dinâmica dos fluídos ou elasticidade, é comum observarmos que a qualidade das aproximações numéricas diminui de forma considerável em regiões contendo singularidades locais ou cuja solução varia de maneira brusca. Para aumentar a qualidade da aproximação nestas regiões é necessário inserir um maior número de pontos na malha de estudo ao redor destas regiões, com o objetivo de reduzir o erro cometido pela aproximação. A principal questão é saber quais trechos do domínio possuem um erro maior, dado que a solução exata é desconhecida.

Inspirado nesta questão, nosso objetivo será construir um estimador de erro utilizando somente a aproximação obtida pelo Método dos Elementos Finitos (MEF) e as informações do contorno. De posse deste estimador, vamos utilizar um algoritmo para particionar o domínio do problema de acordo com os valores fornecidos pelo estimador, ou seja, quanto maior a estimativa do erro cometido numa certa região, maior a quantidade de pontos a serem distribuidos (e consequentemente, maior o número de elementos) nesta região.

Utilizando um estimador de erro a posteriori e um algoritmo de refinamento de malha, a discretização por meio do refinamento adaptativo possui a seguinte estrutura:

- Dada uma discretização inicial e uma tolerância $\varepsilon$ :

I) Obtenha a aproximação pelo MEF;

II) Estime o erro cometido na aproximação;

III) Em cada um dos elementos:

a) Se o erro for maior que a tolerância $\varepsilon$, refine o elemento;

b) Se o erro estimado em todos os elementos for menor que a tolerância $\varepsilon$, encerre.

IV) Se algum elemento foi refinado volte para o item [I].

O enfoque deste trabalho será a construção de um estimador para o erro cometido na aproximação que seja computacionalmente barato em relação ao custo da obtenção de uma nova aproximação e ao mesmo tempo suficientemente robusto para identificar as regiões onde o erro foi maior. Para isto utilizamos o chamado estimador residual, desenvolvido inicialmente nos trabalhos [1] e [2], de L. Demkowicz, J.T. Oden, e T. Strouboulis e nos trabalhos [3] e [4], de R.E. Bank e A. Weiser. Como este estimador é construído de maneira simples a partir das aproximações obtidas e das informações do problema, muitas vezes ele é utilizado como parâmetro de referência para a validação de outros estimadores de erro.

O estudo de estimativas de erro a posteriori aplicadas ao MEF iniciou-se com a construção de estimativas para problemas de valor de contorno unidimensionais, a partir do trabalho [5], de 1978. Em seguida diversas técnicas foram desenvolvidas com a finalidade de estimar o erro cometido na aproximação utilizando a norma induzida pela própria formulação fraca ${ }^{1}$. O desenvolvimento dessas

\footnotetext{
${ }^{1} \mathrm{~A}$ norma associada a forma bilinear da formulação fraca do problema é chamada norma da energia. O assunto será apresentado em detalhes no Capitulo (2).
} 
técnicas serviu como base para a construção dos métodos de medida e controle dos erros obtidos nas aproximações. Durante o período de 1978 até 1984 foram publicados diversos trabalhos importantes na área, dentre os quais os trabalhos [6] e [7] destacam-se por serem obras de referência no estudo de técnicas de estimativa de erro a posteriori.

Outra ideia bastante utilizada na obtenção de estimadores a posteriori é por meio da resolução de sistemas auxiliares em cada uma das partições do domínio, apresentada inicialmente no trabalho [8], de P. Ladevèze and D. Leguillon.

Existem ainda diversos métodos envolvendo extrapolação que podem ser utilizados para obter estimativas globais para o erro, tanto com o refinamento da malha de elementos ( $h$-extrapolation) como com o aumento na ordem das aproximações construídas sobre os elementos ( $p$-extrapolation). No trabalho [10] é construído um estimador bastante eficiente para problemas envolvendo elasticidade linear utilizando sequências de aproximações obtidas por meio de p-exatrapolações.

Para um estudo mais aprofundado sobre as estimativas de erro a posteriori, a obra [11] é uma referência clássica no assunto e condensa as diversas técnicas que podem ser utilizadas na estimação do erro, assim como a obra [13]. O trabalho [12] apresenta também testes comparativos e diferentes aplicações.

Neste trabalho buscamos condensar as principais ideias das obras [13], [14], [15] e [16]. A obra [17], apesar de ser voltada para escoamentos compressíveis em dinâmica dos fluídos, apresenta de forma bastante direta a construção do estimador residual para alguns problemas modelos, dentre eles a Equação de Poisson. As simulações computacionais foram realizadas utilizando o software FreeFem ++ , que tem como objetivo a construção de aproximações numéricas para a solução de equações diferenciais através do MEF. A referência [18] apresenta diversos modelos de códigos, alguns deles aos quais utilizamos como base para a construção das simulações apresentadas aqui.

\section{$1.1 \quad$ Objetivos}

O presente trabalho possui como objetivos principais

- Construir o estimador de erro a residual desenvolvido em [1], [2], [3] e [4], baseado no resíduo entre a aproximação pelo Método dos Elementos Finitos e o problema contínuo;

- Discutir a relação do estimador obtido com o erro cometido na aproximação;

- Apresentar uma possível estratégia para o uso de malhas adaptativas para a redução do erro cometido na aproximação utilizando o estimador residual como função indicadora para o refinamento da malha de elementos utilizada;

- Análise e comparação dos resultados obtidos na aplicação dos refinamentos uniforme e adaptativo em problemas elípticos como a Equação de Poisson e o modelo de Elasticidade Linear

\subsection{Organização do Trabalho}

No Capítulo (2) apresentamos alguns dos conceitos associados ao MEF e, em particular, iremos considerar o método de Ritz-Galerkin. Serão abordadas também as ideias principais de duas estratégias para refinamento das malhas: o refinamento uniforme e o refinamento orientado, além da apresentação do funcionamento do software FreeFEM ++ , utilizado na construção das aproximações utilizadas nos capítulos seguintes.

O Capítulo (3) apresenta a formulação do método para o Problema de Poisson, a construção do estimador residual para este problema e a implementação do estimador e do esquema adaptativo aplicado à um problema cuja solução exata é conhecida e um problema contendo uma singularidade no contorno.

No capítulo (4) generalizamos a teoria aplicada para a solução numérica de problemas envolvendo elasticidade linear e aplicamos o esquema adaptativo utilizando a estimativa residual no problema da placa com furo. 
Por fim, o Capítulo (5) encerra o trabalho apresentando um resumo das conclusões obtidas. No Apêndice (A) apresentamos a demonstração da construção do estimador residual para o problema de elasticidade linear, tratado no Capítulo (4) e no Apêndice (B) encontram-se os códigos utilizados nas simulações. Para facilitar a interpretação dos resultados gráficos optamos por utilizar as figuras do texto em escala reduzida e fornecer no Apêndice (C) as figuras em sua escala original. 


\section{Capítulo 2}

\section{Conceitos, resultados preliminares e obtenção das aproximações}

Neste capítulo serão apresentados alguns resultados teóricos associados à formulação variacional (formulação fraca) das EDP's elípticas e a construção do método de Ritz-Galerkin para a obtenção das aproximações pelo Método dos Elementos Finitos. Numa abordagem mais abstrata, a solução da formulação variacional é vista como a minimização de um dado funcional em um determinado espaço de funções. Desta forma, utilizando técnicas de análise funcional, é possível concluir a existência e a unicidade de soluções em problemas de valor de contorno para equações diferenciais.

Iniciaremos o capítulo apresentando alguns resultados de análise funcional que irão garantir a existência e a unicidade de soluções para os problemas em estudo. Em seguida apresentamos o método de Ritz-Galerkin e discutiremos algumas condições e propriedades das malhas utilizadas e das aproximações obtidas, além de demonstrar o resultado que garante a convergência do método. Apresentaremos uma pequena introdução ao software livre FreeFem ++ , utilizado para obter as aproximações que serão apresentadas nos capítulos seguintes. Encerramos o capítulo apresentando duas possíveis estratégias para o refinamento da malha em estudo: o refinamento uniforme e o refinamento orientado pelo estimador de erro.

As referências [15], [16] e [19] apresentam em detalhes a parte teórica do Método dos Elementos Finitos. Já a referência [18] apresenta o funcionamento completo do FreeFem ++ e discute ambas as técnicas de refinamento da malha de elementos.

\subsection{Ferramentas de análise funcional}

Nesta seção apresentaremos uma série de definições e resultados que serão utilizados com frequência durante o texto.

Definição 2.1.1. Dado $1 \leq p<\infty$, uma função $f: \Omega \subset \mathbb{R}^{n} \mapsto \mathbb{R}$ é dita localmente p-integrável, $f \in L_{l o c}^{p}(\Omega)$ se, para cada $x \in \Omega$ existe uma vizinhança aberta $\Omega^{\prime}$ de $x$ tal que $\overline{\Omega^{\prime}} \subset \Omega$ e $f \in L^{p}\left(\Omega^{\prime}\right)$.

Definição 2.1.2 (Notação multi-índice). Dados $m \in \mathbb{N}$ e $f \in \mathcal{C}^{m}(\Omega)$, denotamos a derivada parcial de ordem $\alpha$ como

$$
D^{\alpha} f=\frac{\partial^{|\alpha|} f}{\partial x_{1}^{\alpha_{1}} \partial x_{2}^{\alpha_{2}} \cdots \partial x_{n}^{\alpha_{n}}}
$$

onde $\alpha \in \mathbb{N}^{n}:|\alpha|=\sum_{i=1}^{n} \alpha_{i} \leq m$

Definição 2.1.3. Dada $v: \Omega \subset \mathbb{R}^{n} \mapsto \mathbb{R}$, dizemos que $v \in \mathcal{C}_{0}^{\infty}(\Omega)$ quando $v$ se anula na fronteira de $\Omega$ e é uma função de classe $\mathcal{C}^{\infty}$ no interior de $\Omega$.

Como exemplo, considere $\Omega=\left\{x \in \mathbb{R}^{n}:|x| \leq 1\right\}$ e a função $v: \Omega \rightarrow \mathbb{R}$ dada por

$$
v(x)= \begin{cases}\exp \left(\frac{1}{|x|^{2}-1}\right) & \text { se }|x|<1 \\ 0 & \text { se }|x| \geq 1\end{cases}
$$


Pode-se mostrar que esta função é de classe $\mathcal{C}^{\infty}(\Omega)$ em todo o espaço ${ }^{1}$ e, pela definição da função, temos que $\left.v\right|_{\partial \Omega}=0$. Logo $v \in \mathcal{C}_{0}^{\infty}(\Omega)$.

Para motivarmos a definição a seguir, considere a função real $f(x)=|x|$ definida no intervalo $[-1,1]$. Apesar da função $f$ não ser diferenciável no ponto $x=0$ gostaríamos de construir um operador semelhante a derivação, no sentido que se a função for de classe $\mathcal{C}^{1}$ a aplicação do operador obtido seja equivalente ao cálculo da derivada da função. Considerando uma função $v \in \mathcal{C}_{0}^{\infty}([-1,1])$ arbitrária, podemos multiplicar $f$ por $v^{\prime}$ e integrar sobre o domínio de definição, de modo que

$$
\int_{-1}^{1} f(x) v^{\prime}(x) d x=\underbrace{\left.f(x) v(x)\right|_{-1} ^{1}}_{=0}-\int_{-1}^{1} f^{\prime}(x) v(x) d x=-\int_{-1}^{1} f^{\prime}(x) v(x) d x
$$

Apesar da função $f$ não ser derivável em todo ponto do intervalo, sua derivada está bem definida para todo ponto diferente da origem. Considerando a função

$$
g(x)=\left\{\begin{array}{cc}
-1 & \text { se } x<0 \\
1 & \text { se } x \geq 0
\end{array}\right.
$$

temos que

$$
\int_{\Omega} f(x) v^{\prime}(x) d x=-\int_{\Omega} g(x) v(x) d x
$$

e $g$ é dita uma derivada fraca da função $f$. Uma vez que a fórmula de Green funciona de forma análoga à integração por partes, a definição a seguir pode ser vista como uma generalização deste resultado para funções em $\mathbb{R}^{n}$.

Definição 2.1.4 (Derivadas fracas). Sejam $\Omega$ um conjunto aberto em $\mathbb{R}^{n}$, não vazio, $f, g \in$ $L_{l o c}^{1}(\Omega)$.Se

$$
\int_{\Omega} f(x) D^{\alpha} v(x) d x=(-1)^{|\alpha|} \int_{\Omega} g(x) v(x) d x \quad \forall v \in \mathcal{C}_{0}^{\infty}
$$

então $g$ é chamada de derivada fraca de ordem $\alpha$ da função $f$.

A definição da derivada fraca de uma função pode ser vista como uma generalização da integral por partes para equações de $\mathbb{R}$ em $\mathbb{R}$ : dado um intervalo real $[a, b]$ e $v \in \mathcal{C}_{0}^{\infty}([a, b])$, se existe uma função $g$ tal que $g=f^{\prime}$ em quase todo ponto do intervalo $[a, b]$ (ou seja, $g(x) \neq f^{\prime}(x)$ somente em um conjunto enumerável de pontos) temos que

$$
\int_{\Omega} f(x) v^{\prime}(x) d x=\underbrace{\left.f(x) v(x)\right|_{a} ^{b}}_{=0}-\int_{\Omega} f^{\prime}(x) v(x) d x=-\int_{\Omega} g(x) v(x) d x
$$

Logo, dizemos que $g$ é uma derivada fraca da função $f$.

Um questionamento natural neste ponto é sobre a unicidade da derivada fraca de uma função. Para demonstrarmos este resultado, considere o seguinte Lema:

Lema 2.1.5. Sejam $\Omega$ um aberto não vazio de $\mathbb{R}^{n}$ e $u \in L_{l o c}^{1}(\Omega)$. Se

$$
\int_{\Omega} u v=0, \quad \forall v \in C_{0}^{\infty}(\Omega)
$$

então $u=0$ em $\Omega$, a menos de um conjunto de medida nula.

De posse deste resultado, vamos a seguinte proposição

Proposição 2.1.6 (Unicidade da derivada fraca). Sejam $\Omega$ um conjunto aberto não vazio em $\mathbb{R}^{n}$ e $f \in L_{l o c}^{1}(\Omega)$. Se $f$ possui derivada fraca então a derivada fraca é única, a menos de um conjunto de medida nula.

\footnotetext{
${ }^{1}$ Veja em [15], exemplo 1.2.2 - pág 27
} 
Demonstração. Por absurdo, suponha que $f$ admita duas derivadas fracas de uma certa ordem $\alpha$, digamos $g_{1}$ e $g_{2}$. Pela definição das derivadas fracas

$$
\begin{aligned}
& \int_{\Omega} f(x) D^{\alpha} v(x) d x=(-1)^{|\alpha|} \int_{\Omega} g_{1}(x) v(x) d x \\
& \int_{\Omega} f(x) D^{\alpha} v(x) d x=(-1)^{|\alpha|} \int_{\Omega} g_{2}(x) v(x) d x
\end{aligned}
$$

Subtraindo as equações, temos que

$$
\int_{\Omega}\left(g_{1}(x)-g_{2}(x)\right) v(x) d x=0
$$

onde, pelo Lema (2.1.5), temos que $g_{1}=g_{2}$ a menos de um conjunto de medida nula.

Apresentaremos a seguir um espaço de funções muito importante que será utilizado com frequência na construção das aproximações pelo Método dos Elementos Finitos:

Definição 2.1.7 (Espaços $H^{k}$ ). Dados $\Omega \subset \mathbb{R}^{n}$ e $k \in \mathbb{N}$, definimos

$$
H^{k}(\Omega)=\left\{f \in L^{2}(\Omega): \forall \alpha:|\alpha| \leq k,\left\|D^{\alpha} f\right\|_{L^{2}}=\left(\int_{\Omega}\left(D^{\alpha} f\right)^{2}\right)^{\frac{1}{2}}<\infty\right\}
$$

ou seja, o espaço das funções $L^{2}(\Omega)$ tal que todas as derivadas fracas até a ordem $k$ estão em $L^{2}(\Omega)$.

Considerando o produto interno em $H^{k}$ dado por

$$
<u, v>=\sum_{|\alpha|<k}<D^{\alpha} u \cdot D^{\alpha} v>_{L^{2}}=\sum_{|\alpha|<k} \int_{\Omega} D^{\alpha} u \cdot D^{\alpha} v
$$

podemos definir a norma neste espaço como

$$
\|u\|_{H^{k}(\Omega)}=\sqrt{<u, u>}=\left(\sum_{|\alpha|<k}\left\|D^{\alpha} u\right\|_{L^{2}(\Omega)}^{2}\right)^{\frac{1}{2}}
$$

Quando não houver risco de ambiguidade em relação ao domínio $\Omega$, vamos considerar $\|u\|_{H^{k}(\Omega)}=$ $\|u\|_{k}$.

Proposição 2.1.8. Nas condições da definição anterior, temos que o espaço $H^{k}(\Omega)$ é um espaço de Hilbert.

Teorema 2.1.9 (Desigualdade de Poincaré). Seja $\Omega \subset \mathbb{R}^{n}$ um conjunto aberto e limitado, com fronteira seccionalmente diferenciavel e $u \in H_{0}^{1}(\Omega)$. Então, vale a desigualdade

$$
\|u\|_{L^{2}(\Omega)} \leq C\|\nabla u\|_{L^{2}(\Omega)}
$$

onde a constante $C$ depende apenas do dominio $\Omega$.

Teorema 2.1.10. Seja $\Omega \subset \mathbb{R}^{n}$ um aberto com fronteira seccionalmente diferenciavel $e k \in \mathbb{N}$. Então $C^{\infty}(\Omega) \cap H^{k}(\Omega)$ é denso em $H^{k}(\Omega)$.

A demonstração destes resultados utiliza algumas definições que não serão apresentadas aqui, mas podem ser encontradas em [15], [16], [19], [20] ou diversas outras referências sobre espaços de Sobolev. 
Definição 2.1.11 (Forma bilinear). Dado $V$ um espaço vetorial, dizemos que um operador $a$ é uma forma bilinear se, fixado $u \in V$, temos que $v \mapsto a(u, v)$ e $v \mapsto a(v, u)$ são operadores lineares em $V$. Além disso, se $a(u, v)=a(v, u)$, a forma bilinear é dita simétrica.

Definição 2.1.12 (Continuidade e coercividade). Uma forma bilinear $a(\cdot, \cdot)$ num espaço normado $V$ é dita limitada ou continua se $\exists C<\infty$ tal que

$$
|a(u, v)| \leq C|| u\||\cdot| \mid v\| \quad \forall u, v \in V
$$

e é dita coerciva em $W \subset V$ se $\exists \alpha>0$ tal que

$$
a(w, w) \geq \alpha\|w\|^{2} \quad \forall w \in W
$$

onde $\|\cdot\|$ representa a norma de $\mathrm{V}$.

Proposição 2.1.13. Seja $H$ um espaço de Hilbert, $W$ um subespaço fechado de $H$ e a $(\cdot, \cdot)$ uma forma bilinear simétrica contínua em $H$ e coerciva em $W$. Então, $(W, a(\cdot, \cdot))$ é um espaço de Hilbert.

Demonstração. Da coercividade da forma bilinear $a$ temos que se $w \in W$ e $a(w, w)=0$, então $w=0$. Logo, $a(\cdot, \cdot)$ define um produto interno em $W$. Seja $\|w\|_{E}=\sqrt{a(w, w)}$ e considere $w_{n}$ uma sequência de Cauchy em $\left(W,\|\cdot\|_{E}\right)$. Pela coercividade $w_{n}$ é uma sequência de Cauchy em $\left(H,\|\cdot\|_{H}\right)$. Como $H$ é completo, $\exists w \in H: w_{n} \rightarrow w$. Sendo $W$ fechado em $H$, temos que $w \in W$. Mas

$$
\left\|w-w_{n}\right\|_{E} \leq \sqrt{C}\left\|w-w_{n}\right\|_{H}
$$

pois $a(\cdot, \cdot)$ é continua. Logo, $w_{n} \rightarrow w$ na norma $\|\cdot\|_{E}$ e, portanto $\left(W,\|\cdot\|_{E}\right)$ é completo.

Teorema 2.1.14. Suponha que sejam válidas as seguintes condições

1. $(H,(\cdot, \cdot))$ é um espaço de Hilbert

2. $V$ é um subespaço fechado de $H$

3. $a(\cdot, \cdot)$ é uma forma bilinear simétrica, contínua em $H$ e coerciva em $V$.

Então, dado um funcional linear $f \in V^{\prime}$, existe uma única $u \in V$ tal que:

$$
a(u, v)=<f, v>, \quad \forall v \in V
$$

Demonstração. Como $a(\cdot, \cdot)$ é um produto interno em $V$ e $(V, a(\cdot, \cdot))$ é um espaço de Hilbert, o resultado segue da aplicação do Teorema da Representação de Riesz ${ }^{2}$.

Os problemas apresentados neste trabalho apresentam suas formas bilineares todas simétricas porém, os resultados podem ser aplicados aos casos em que a forma bilinear a não é simétrica por meio do seguinte resultado:

Teorema 2.1.15 (Lax-Milgram). Dados um espaço de Hilbert $(V,(\cdot, \cdot))$, uma forma bilinear contínua e coerciva $a(\cdot, \cdot)$ e $f \in V^{\prime}$, existe um único $u \in V$ satisfazendo a relação (2.10).

A demonstração deste resultado é feita reescrevendo-se o problema como um problema de ponto fixo e aplicando-se o teorema do ponto fixo de Banach. Para o leitor interessado, a demonstração encontra-se completa em [15].

\footnotetext{
${ }^{2} \mathrm{O}$ teorema da representação de Riesz afirma que, dado um espaço de Hilbert V e um funcional $f: V \mapsto \mathbb{R}$, existe um único $u \in V$ tal que para todo $v \in V$ temos que $f(v)=\langle u, v\rangle$, onde $\langle\cdot, \cdot\rangle$ é o produto interno em V. A demonstração deste resultado pode ser obtida em [19] ou outras referências de análise funcional.
} 


\subsection{Formulação Equivalente}

O problema anterior pode ser analisado numa formulação equivalente, através do estudo do ponto de mínimo do funcional associado ao problema, como mostra o teorema a seguir:

Teorema 2.2.1 (Formulação Equivalente). Seja $V$ um espaço vetorial normado, $a: V \times V \rightarrow \mathbb{R}$ uma forma bilinear simétrica positiva (isto é, $a(v, v)>0, \forall v \in V, v \neq 0$ ), limitada e coerciva. Considere ainda $f \in V^{\prime}$. Então, o funcional dado por

$$
J(v)=\frac{1}{2} a(v, v)-<f, v>
$$

atinge seu minimo em $u \in V$ se, e somente se

$$
a(u, v)=<f, v>, \forall v \in V
$$

Demonstração. Para todo $u, v \in V$ e $t \in \mathbb{R}$ temos que

$$
\begin{aligned}
J(u+t v) & =\frac{1}{2} a(u+t v, u+t v)-<f, u+t v> \\
& =J(u)+t[a(u, v)-<f, v>]+\frac{t^{2}}{2} a(v, v)
\end{aligned}
$$

Se $u \in V$ satisfaz (2.12), então para $t=1$ temos que

$$
J(u+t v)=J(u)+\underbrace{\frac{1}{2} a(v, v)}_{>0}>J(u), \quad \forall v \in V: v \neq 0
$$

concluindo assim que $u$ é ponto de mínimo. Por outro lado, se $J$ admite valor mínimo em $u$, para cada $v \in V$, a derivada do operador $t \mapsto J(u+t v)$ deve ser nula em $t=0$. Por (2.13), a derivada deste operador ser nula equivale a condição $(2.12)$, concluindo assim o resultado desejado.

\subsection{Problemas de valor de contorno}

Dado um domínio $\Omega$, para que uma EDP elíptica possua solução única é necessária a prescrição de informações sobre a solução no contorno do domínio. A definição a seguir apresenta duas possíveis condições $^{3}$ e apresenta a notação que será utilizada em cada um destes trechos.

Definição 2.3.1 (Condições de Fronteira). Sejam $\Omega \subset \mathbb{R}^{n}$ um aberto e $\Gamma$ a sua fronteira. Denotaremos o trecho da fronteira onde é conhecida a solução do problema de valor de contorno por $\Gamma_{D}$ e o trecho da fronteira onde é conhecida a derivada normal da solução por por $\Gamma_{N}$.

A nomenclatura destes conjuntos está diretamente ligada com a descrição utilizada nos problemas de valor de contorno: o conjunto $\Gamma_{D}$ remete as condições de Dirichlet enquanto o conjunto $\Gamma_{N}$ refere-se as condições de Neumann associada ao problema.

Para os trechos onde são conhecidas as condições de Neumann do problema será necessário o uso de uma função capaz de restingir a solução na fronteira de maneira limitada. O resultado a seguir garante a existência de um operador com esta propriedade.

Teorema 2.3.2 (Teorema do traço). Seja $\Omega$ um conjunto poligonal limitado. Então, existe um operador linear limitado

$$
\gamma: H^{1}(\Omega) \rightarrow L^{2}(\Gamma):\|\gamma(u)\|_{L^{2}(\Gamma)} \leq\|u\|_{1}
$$

tal que $\gamma(u)=\left.u\right|_{\partial \Omega}$ para toda $u \in \mathcal{C}^{1}(\bar{\Omega})$

\footnotetext{
${ }^{3}$ Neste trabalho não iremos trabalhar com as chamadas condições de Robin, que consistem em um trecho da fronteira onde é conhecida uma combinação da solução e da derivada normal.
} 
Este resultado garante dada uma função $u \in H^{1}$, sua restrição a fronteira será pelo menos uma função contida em $L^{2}(\Gamma)$. A demonstração deste resultado é bastante técnica e encontra-se em [16].

Encerramos esta seção definindo os espaços de funções sobre os quais serão construídos os espaços de dimensão finita para a construção das aproximações:

Definição 2.3.3. Nas condições das definições (2.1.7) e (2.3.1), denotaremos por $H_{0}^{1}(\Omega)$ e $H_{D}^{1}(\Omega)$ as funções em $H^{1}(\Omega)$ que se anulam em $\Gamma$ e em $\Gamma_{D}$, respectivamente. Denotamos também o conjunto $H^{\frac{1}{2}}(\Gamma)$ composto pelas funções $\psi$ em $L^{2}(\Gamma)$ tais que $\psi=\left.\phi\right|_{\Gamma}$ para alguma $\phi \in H^{1}(\Omega)$.

\subsection{Método de Ritz-Galerkin}

A idéia do método de Ritz-Galerkin é substituir o espaço $H_{D}^{1}(\Omega)$ por um espaço de dimensão finita $V_{h} \subset H_{D}^{1}(\Omega)$ associado a um determinado conjunto de pontos do domínio, de forma a obter uma aproximação $u_{h} \in V_{h}$ tal que:

$$
a\left(u_{h}, v_{h}\right)=<f, v_{h}>, \quad \forall v \in V_{h}
$$

onde o subscrito $h$ remete ao espaçamento que da malha que será relacionada com o espaço de dimensão finita.

Uma importante propriedade do Método de Ritz-Galerkin é o resultado a seguir:

Lema 2.4.1 (Ortogonalidade de Galerkin). Sejam $u \in H_{D}^{1}(\Omega)$ e $u_{h} \in V_{h}$ as soluçôes de (2.10) $e$ (2.15), respectivamente. Então:

$$
a\left(u-u_{h}, v_{h}\right)=0
$$

para toda função $v_{h} \in V_{h}$.

Demonstração. Como $V_{h} \subset H_{D}^{1}(\Omega)$ podemos escolher $v=v_{h}$ em (2.10) e subtrair (2.15), de forma que

$$
\begin{gathered}
a\left(u, v_{h}\right)-a\left(u_{h}, v_{h}\right)=<f, v_{h}>-<f, v_{h}>=0 \\
\Rightarrow a\left(u-u_{h}, v_{h}\right)=0
\end{gathered}
$$

Este resultado indica que o erro cometido na aproximação pelo método de Galerkin é ortogonal ao subespaço $V_{h}$ em relação ao produto interno induzido pelo operador $a$. Ou seja, a solução obtida pela método de Galerkin é exatamente a projeção ortogonal da solução exata no subespaço $V_{h}$.

O espaço de dimensão finita $V_{h} \subset H_{D}^{1}(\Omega)$ é obtido através do particionamento do domínio em um número finito de elementos que apresentam as mesmas propriedades do domínio completo. Pela definição de Ciarlet [21]:

Definição 2.4.2 (Elemento Finito). Seja

i) $K \subset \mathbb{R}^{n}$ um conjunto fechado e limitado, com interior não vazio e fronteira suave por partes (chamado elemento).

ii) $\mathcal{P}$ um espaço de dimensão finita de funções definidas sobre $K$ (espaço das funções de forma ou shape-functions).

iii) $\mathfrak{N}_{K}$ uma base para $\mathcal{P}$ (chamado conjunto das variaveis nodais).

Então $\left(K, \mathcal{P}, \mathfrak{N}_{K}\right)$ é chamado um elemento finito.

Em geral, utilizam-se triangulos e quadriláteros para problemas em $\mathbb{R}^{2}$ e tetrahedros e paralelepípedos para problemas em $\mathbb{R}^{3}$. Já as funções de forma podem ser definidas por polinômios ou funções trigonométricas e relacionam-se com o conjunto de variáveis nodais pela propriedade de que cada função de forma é não nula em apenas um dos nós. 


\subsubsection{Partições do domínio}

Considerando $\Omega \subset \mathbb{R}^{2}$ um conjunto poligonal e uma discretização de $\Omega$ composta por triângulos, vamos a seguinte definição:

Definição 2.4.3 (Partição admissível). Uma partição (ou discretização) $\mathcal{T}$ do domínio $\Omega$ é dita admissivel se

i) $\bigcup_{K \in \mathcal{T}} K=\Omega \cup \Gamma$.

ii) Se $K, K^{\prime} \in \mathcal{T}: K \cap K^{\prime}=x, x \in \bar{\Omega}$, então $x$ é um vértice de $K$ e de $K^{\prime}$

iii) Dados dois elementos $K_{1}$ e $K_{2}$ se a intersecção entre eles for maior que um ponto, então $K_{1} \cap K_{2}$ é uma aresta (ou uma face) em comum entre $K_{1}$ e $K_{2}$

As condições acima garantem que todos os pontos da malha sejam nós dos elementos, de forma que nenhuma aresta possui um nó que não esteja em seus extremos. A Figura (2.1) contém exemplos de malhas admissíveis e malhas não-admissíveis.

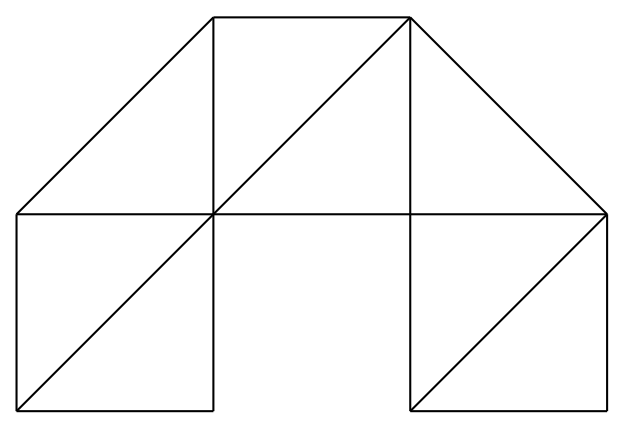

(a)

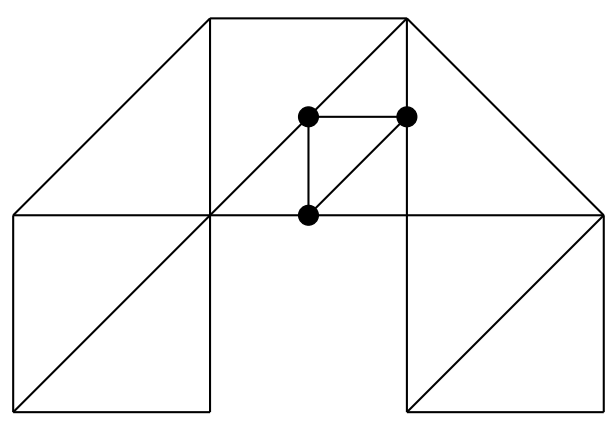

(b)

Figura 2.1: Exemplos de malhas de um dominio $\Omega$. A figura a) mostra uma malha admissivel enquanto que a figura b) mostra uma malha não admissivel, pois possui um elemento cujos vértices estão situados nos pontos médios das arestas de outros elementos.

Definição 2.4.4. Para cada partição admissível $\mathcal{T}$, definimos os seguintes conjuntos

- $N_{\mathcal{T}}$ : Conjunto dos vértices de todos os elementos da partição.

- $\mathcal{E}_{\tau}$ : Conjunto das arestas (ou faces) de todos os elementos da partição.

- $N_{\mathcal{T}, \Gamma_{D}}$ : Vértices contidos no contorno $\Gamma_{D}$.

- $N_{\mathcal{T}, \Gamma_{N}}$ : Vértices contidos no contorno $\Gamma_{N}$.

- $N_{\mathcal{T}, \Omega}$ : Vértices contidos no interior do domínio.

- $\mathcal{E}_{\tau, \Gamma_{D}}:$ Arestas (ou faces) contidas no contorno $\Gamma_{D}$.

- $\mathcal{E}_{\tau, \Gamma_{N}}$ : Arestas (ou faces) contidas no contorno $\Gamma_{N}$.

- $\mathcal{E}_{\tau, \Omega}$ : Arestas (ou faces) contidas no interior do domínio. 
Definição 2.4.5. Dada uma partição $\mathcal{T}$, sejam $K, E$ e $x$ um elemento, uma aresta e um ponto da partição, respectivamente. Definimos então os conjuntos

- $\omega_{K}=\bigcup_{\mathcal{E}_{K} \cap \mathcal{E}_{K^{\prime}} \neq \emptyset} K^{\prime}$ : Conjunto dos elementos que são vizinhos de $K$.

- $\tilde{\omega}_{K}=\bigcup_{N_{K} \cap N_{K^{\prime}} \neq \emptyset} K^{\prime}$ : Conjunto dos elementos que possuem pontos em comum com o elemento $K$.

- $\omega_{E}=\bigcup_{E \in E_{K^{\prime}}} K^{\prime}$ : Conjunto dos elementos que possuem a aresta $E$

- $\tilde{\omega}_{E}=\bigcup_{N_{E} \cap N_{K^{\prime}} \neq \emptyset} K^{\prime}$ : Conjunto dos elementos que são vizinhos aos elementos que possuem a aresta $E$.

- $\omega_{x}=\bigcup_{x \in N_{K^{\prime}}} K^{\prime}$ : Conjunto dos elementos que possuem o ponto $x$ como vértice.

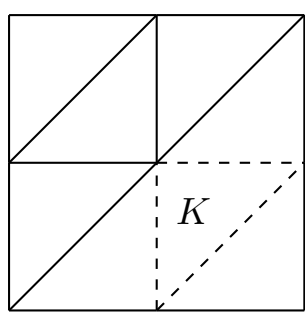

(a)

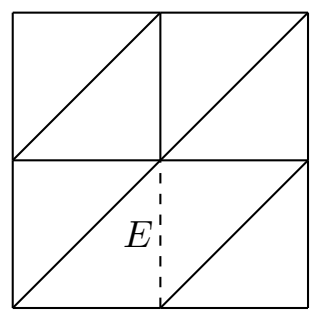

(d)

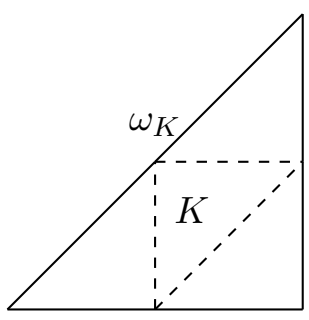

(b)

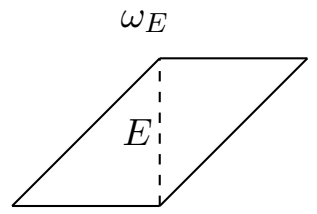

(e)

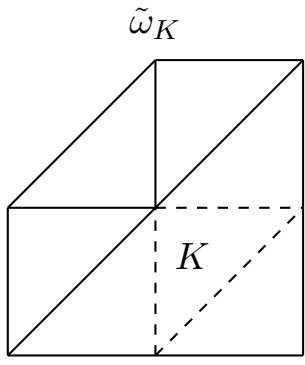

(c)

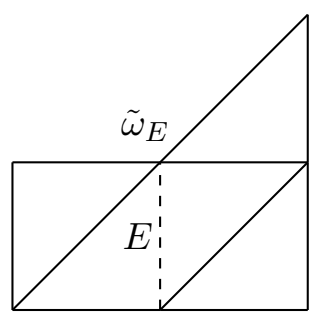

(f)

Figura 2.2: Exemplos dos conjuntos apresentados na definição (2.4.5).

Considerando uma partição admissível do domínio, gostaríamos de definir uma medida para a malha utilizada através de algum parâmetro relacionado a discretização.

Definição 2.4.6 (Partição shape-regular). Considere $\mathcal{T}$ uma partição do domínio, $K$ um elemento da partição, $h_{K}$ o diâmetro do elemento e $\rho_{K}$ o diâmetro da maior bola contida em $K$. Dado $\delta \in \mathbb{R}$, dizemos que uma partição é de forma regular (shape-regular) se

$$
C_{K}=\frac{h_{K}}{\rho_{K}}<\delta
$$

para todo $K \in \mathcal{T}$. Definimos o parâmetro de refinamento da partição $\mathcal{T}$ como

$$
C_{\mathcal{T}}=\max _{K \in \mathcal{T}} \frac{h_{K}}{\rho_{K}}
$$




\subsubsection{Funções de forma e funções teste}

Definição 2.4.7 (Conjunto $\mathbb{P}^{k}$ ). Dado $k \in \mathbb{N}$, definimos $\mathbb{P}^{k}$ como o conjunto dos polínômios de grau menor ou igual a $k$.

Definição 2.4.8 (Conjunto $S_{D}^{k, 0}(\mathcal{T})$ ). Seja $\mathcal{T}$ uma partição por elementos do domínio. Associamos à essa partição o espaço $S^{k}(\mathcal{T})$, composto pelas funções polinomiais de ordem menor ou igual a $k$ no interior de cada um dos elementos da partição e contínua no bordo dos elementos, isto é

$$
\left.\psi \in S^{k}(\mathcal{T}) \Rightarrow \psi\right|_{K} \in \mathbb{P}^{k} \text {, para todo } K \in \mathcal{T} \text { e } \psi \in \mathcal{C}(\bar{K})
$$

Para obtermos as aproximações do método de Ritz-Galerkin, consideramos o espaço

$$
S_{D}^{k, 0}(\mathcal{T})=S^{k}(\mathcal{T}) \cap H_{D}^{1}(\Omega)=\left\{\psi \in S^{k}(\mathcal{T}): \psi=0 \text { em } \Gamma_{D}\right\}
$$

Desta forma, substituindo $V_{h}$ por $S_{D}^{1,0}(\mathcal{T})$ em $(2.15)$ obtemos como aproximação a projeção da solução $u \in H_{D}^{1}(\Omega)$ sobre o espaço de dimensão finita dado pela partição $\mathcal{T}$ do domínio $\Omega$.

Para cada um dos elementos $K \in \mathcal{T}$, a restrição da solução sobre $K$ será feita utilizando a chamada Base de Lagrange em cada um dos pontos que compõem o elemento. Dado $x \in N_{\mathcal{T}}$, a função de forma nodal associada a este ponto consiste em um determinado polinômio $\lambda_{x}$, definido sobre os elementos $K: x \in N_{K}$ e tal que $\lambda_{x}=1$ no vértice $x$ e $\lambda_{x}=0$ nos outros vértices. Desta forma, dada uma função $f \in \mathcal{C}(\bar{\Omega})$, a interpolação de $\mathrm{f}$ sobre os vértices de $K$ pode ser escrita como uma combinação linear das funções de forma:

$$
J_{\mathcal{T}} f=\sum_{i=0}^{N-1} f\left(n_{i}\right) \lambda_{n_{i}}(x)
$$

onde $N$ é a quantidade de vértices do elemento $K$. Na literatura de elementos finitos, a aproximação obtida sobre o domínio $\Omega$ é chamada de função teste.

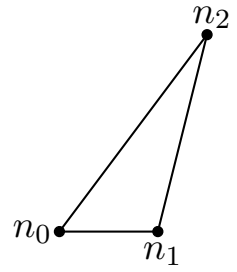

(a)

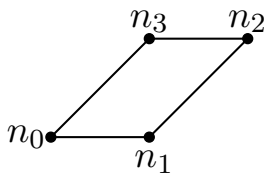

(b)

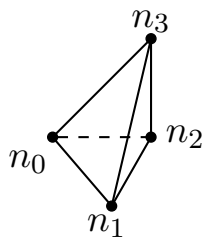

(c)

Figura 2.3: As figuras (a) e (b) mostram elementos lineares em $\mathbb{R}^{2}$ e a figura (c) apresenta o elemento linear em $\mathbb{R}^{3}$.

Vamos apresentar a construção das funções de forma para alguns elementos:

a) Considere um triângulo $\mathrm{K}$ com vértices $n_{0}, n_{1}$ e $n_{2}$ numerados no sentido anti-horário, como descrito na Figura (2.3a). Para cada um dos vértices $n_{i}, i=0,1,2$ a função de forma associada ao vértice é dada por

$$
\lambda_{n_{i}}(x)=\frac{\operatorname{det}\left(x-n_{i+1}, n_{i+2}-n_{i+1}\right)}{\operatorname{det}\left(n_{i}-n_{i+1}, n_{i+2}-n_{i+1}\right)}, \quad i=0,1,2
$$

onde os índices utilizados em $\lambda_{n_{i}}$ são considerados em módulo 3 .

b) Utilizando um paralelogramo K com vértices $n_{0}, n_{1}, n_{2}$ e $n_{3}$ numerados no sentido anti-horário, como descrito na Figura (2.3b), a função de forma associada a cada um dos vértices é dada 
por

$$
\lambda_{n_{i}}(x)=\frac{\operatorname{det}\left(x-n_{i+2}, n_{i+3}-n_{i+2}\right)}{\operatorname{det}\left(n_{i}-n_{i+2}, n_{i+3}-n_{i+2}\right)} \cdot \frac{\operatorname{det}\left(x-n_{i+2}, n_{i+1}-n_{i+2}\right)}{\operatorname{det}\left(n_{i}-n_{i+2}, n_{i+1}-n_{i+2}\right)}, \quad i=0,1,2,3
$$

onde os índices utilizados em $\lambda_{n_{i}}$ são considerados em módulo 4.

c) No caso do tetrahedro $\mathrm{K}$ com vértices $n_{0}, n_{1}, n_{2}$ e $n_{3}$ enumerados como na Figura $(2.3 \mathrm{c})$ a função de forma associada a cada um dos vértices é dada por

$$
\lambda_{n_{i}}(x)=\frac{\operatorname{det}\left(x-n_{i+1}, n_{i+2}-n_{i+1}, n_{i+3}-n i+1\right)}{\operatorname{det}\left(n_{i}-n_{i+1}, n_{i+2}-n_{i+1}, n_{i+3}-n_{i+1}\right)}, \quad i=0,1,2,3
$$

onde os índices utilizados em $\lambda_{n_{i}}$ são de módulo 4.

\subsubsection{Operador de quasi-interpolação}

Dado que o operador padrão de interpolação $J_{\mathcal{T}}$ apresentado na seção anterior só faz sentido quando aplicado em funções contínuas e o espaço $H^{1}$ admite funções descontínuas, será necessário a utilização de um operador de interpolação mais fraco para a construção do estimador, conhecido como operador de quasi-interpolação ou operador de Clèment.

Definição 2.4.9 (Operador de Clèment). Considere o operador $I_{\mathcal{T}}: L^{1}(\Omega) \rightarrow S_{D}^{1,0}(\mathcal{T})$ definido por

$$
I_{\mathcal{T}} v=\sum_{x \in N_{\mathcal{T}, \Omega} \cup N_{\mathcal{T}, \Gamma_{N}}} \lambda_{x} \frac{1}{\left|\omega_{x}\right|} \int_{\omega_{x}} v
$$

onde $\left|\omega_{x}\right|$ representa a área $\left(\mathrm{em} \mathbb{R}^{2}\right)$ ou o volume $\left(\mathrm{em} \mathbb{R}^{3}\right)$ do conjunto $\omega_{x}$.

Apesar deste operador não interpolar de fato a função $v \in L^{1}(\Omega)$ nos pontos $x \in N_{\mathcal{T}}$, sua principal característica é que o erro cometido na interpolação depende somente da malha utilizada na região onde o operador foi aplicado, como mostra o resultado a seguir:

Proposição 2.4.10. Dada $\mathcal{T}$ uma partição admissível do domínio, o operador de Clément satisfaz as estimativas

$$
\begin{aligned}
\left\|v-I_{\mathcal{T}} v\right\|_{L^{2}(K)} & \leq \alpha h_{K}\|v\|_{H^{1}\left(\tilde{\omega}_{K}\right)} \\
\left\|v-I_{\mathcal{T}} v\right\|_{L^{2}(\partial K)} & \leq \beta \sqrt{h_{E}}\|v\|_{H^{1}\left(\tilde{\omega}_{E}\right)}
\end{aligned}
$$

para toda $v \in H_{D}^{1}$ e para todos os elementos $K \in \mathcal{T}$, onde $h_{K}$ representa o diâmetro do elemento $K, h_{E}$ representa o diâmetro da aresta (ou face) $E$ e as constantes $\alpha$ e $\beta$ dependem apenas do parâmetro de refinamento de malha $C_{\mathcal{T}}$.

Apesar de ser bastante simples, a demonstração deste resultado envolve alguns resultados que não serão apresentados aqui, como o Lema de Bramble-Hilbert. A demonstração deste resultado encontra-se em [16], pág 84.

\subsubsection{Função de salto (jump)}

$\mathrm{Na}$ avaliação da estimativa residual sobre cada um dos elementos do domínio será necessário avaliar a diferença que ocorre entre os gradientes de dois elementos adjacentes. Para isto, vamos a seguinte definição:

Definição 2.4.11 (Função de Salto). Dados dois elementos adjacentes $K$ e $K^{\prime}$ conectados pela aresta $E \in \mathcal{E}_{\tau, \Omega}$, consideramos o salto ocorrido em $E$ como:

$$
\mathbb{J}_{E}\left(\vec{n}_{E} \cdot \nabla u_{\tau}\right)(x)=\lim _{\epsilon \rightarrow 0}\left[\left.\nabla u_{\tau}\right|_{K}\left(x+\epsilon \vec{n}_{E}\right)-\left.\nabla u_{\tau}\right|_{K^{\prime}}\left(x-\epsilon \vec{n}_{E}\right)\right]
$$


onde $\epsilon \in \mathbb{R}, x \in E$ e $\vec{n}_{E}$ é o vetor normal exterior a aresta $E$. Como todos os elementos possuem os vértices enumerados no sentido anti-horário, a normal exterior ao elemento $K^{\prime}$ na aresta $E$ equivale a normal exterior ao elemento $K$ na aresta $E$ com o sinal negativo.

No caso onde forem utilizados elementos triangulares e as funções de forma forem polinômios de primeiro grau, o gradiente em cada um dos elementos adjacentes será constante sobre cada um dos elementos. Neste caso, a função de salto é simplificada como

$$
\mathbb{J}_{E}\left(\vec{n}_{E} \cdot \nabla u_{\tau}\right)(x)=\frac{1}{2}\left(\nabla u_{\tau}^{K}-\nabla u_{\tau}^{K^{\prime}}\right) \cdot \vec{n}_{E}
$$

onde $\nabla u_{\tau}^{K}$ e $\nabla u_{\tau}^{K^{\prime}}$ são os gradientes nos elementos $K$ e $K^{\prime}$ que possuem a aresta $E$ em comum.

\subsubsection{Construção das aproximações}

Considerando o elemento triangular com três nós apresentado na Figura (2.4), observamos que em cada um dos elementos os nós do elemento são posicionados nas extremidades do triângulo e denotados por $\left(x_{i}^{e}, y_{i}^{e}\right)$, com $i=1,2,3$.

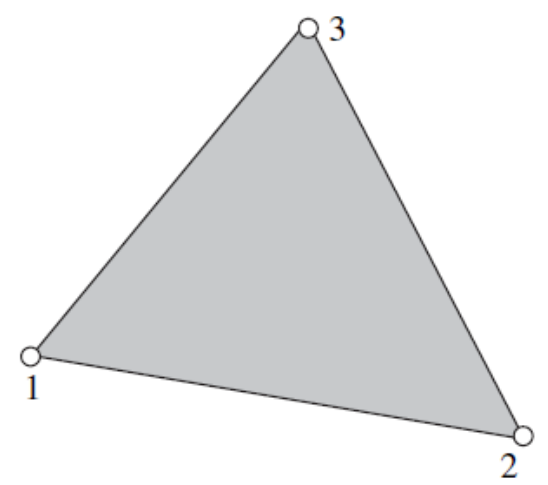

Figura 2.4: Elemento triangular de três nós

Para um dado elemento, a aproximação no interior deste elemento é da forma

$$
u^{e}(x, y)=\alpha_{0}^{e}+\alpha_{1}^{e} x+\alpha_{2}^{e} y=\mathbf{N}^{e}(x, y) \alpha^{e}
$$

onde $\alpha^{e}$ é o vetor com as aproximações nodais e

$$
\mathbf{N}^{e}(x, y)=\underbrace{\frac{1}{2 A^{e}}\left[\begin{array}{lll}
\left(x_{2}^{e} y_{3}^{e}-x_{3}^{e} y_{2}^{e}\right) & \left(y_{23}^{e}\right) & \left(x_{32}^{e}\right) \\
\left(x_{3}^{e} y_{1}^{e}-x_{1}^{e} y_{3}^{e}\right) & \left(y_{31}^{e}\right) & \left(x_{13}^{e}\right) \\
\left(x_{1}^{e} y_{2}^{e}-x_{2}^{e} y_{1}^{e}\right) & \left(y_{12}^{e}\right) & \left(x_{21}^{e}\right)
\end{array}\right]}_{\mathbf{M}^{e}}\left[\begin{array}{l}
1 \\
x \\
y
\end{array}\right]
$$

são as funções de forma para cada um dos nós dos elementos (ou seja, são as funções de interpolação definidas sobre o elemento e) e $A^{e}$ representa a área do elemento em estudo.

Considerando as aproximações no interior do elemento escritas como

$$
\begin{gathered}
u^{e}(x, y)=\mathbf{N}^{e}(x, y)\left(\alpha^{e}\right)^{T} \Rightarrow \nabla u^{e}(x, y)=\mathbf{B}^{e}(x, y)\left(\alpha^{e}\right)^{T} \\
w^{e}(x, y)=\mathbf{N}^{e}(x, y)\left(\mathbf{w}^{e}\right)^{T} \Rightarrow \nabla w^{e}(x, y)=\mathbf{B}^{e}(x, y)\left(\mathbf{w}^{e}\right)^{T}
\end{gathered}
$$


onde o gradiente das funções teste é dado por:

$$
\begin{aligned}
\nabla u^{e}(x, y) & =\left[\begin{array}{c}
\frac{\partial u^{e}(x, y)}{\partial x} \\
\frac{\partial u^{e}(x, y)}{\partial y}
\end{array}\right] \\
& =\left[\begin{array}{c}
\frac{\partial N_{1}^{e}}{\partial x} \alpha_{1}^{e}+\frac{\partial N_{2}^{e}}{\partial x} \alpha_{2}^{e}+\frac{\partial N_{3}^{e}}{\partial x} \alpha_{3}^{e} \\
\frac{\partial N_{1}^{e}}{\partial y} \alpha_{1}^{e}+\frac{\partial N_{2}^{e}}{\partial y} \alpha_{2}^{e}+\frac{\partial N_{3}^{e}}{\partial y} \alpha_{3}^{e}
\end{array}\right] \\
& =\frac{1}{2 A^{e}}\left[\begin{array}{lll}
y_{23}^{e} & y_{31}^{e} & y_{12}^{e} \\
x_{32}^{e} & x_{13}^{e} & x_{21}^{e}
\end{array}\right]\left[\begin{array}{c}
\alpha_{1}^{e} \\
\alpha_{2}^{e} \\
\alpha_{3}^{e}
\end{array}\right]
\end{aligned}
$$

$\operatorname{com} x_{i, j}^{e}=x_{i}^{e}-x_{j}^{e}$ e $y_{i, j}^{e}=y_{i}^{e}-y_{j}^{e}$, da continuídade entre dois elementos adjacentes temos que

$$
\sum_{e=1}^{n_{e l}}\left(\mathbf{w}^{e}\right)^{T}\left[\mathbf{K}^{e} \alpha^{e}-f^{e}\right]=0
$$

onde

$$
\mathbf{K}^{e}=\int_{\Omega^{e}}\left(\mathbf{B}^{e}\right)^{T} \mathbf{D} B^{e}=\left[\begin{array}{ccc}
\mathbf{K}_{11}^{e} & \mathbf{K}_{12}^{e} & \mathbf{K}_{13}^{e} \\
\mathbf{K}_{21}^{e} & \mathbf{K}_{22}^{e} & \mathbf{K}_{23}^{e} \\
\mathbf{K}_{31}^{e} & \mathbf{K}_{32}^{e} & \mathbf{K}_{33}^{e}
\end{array}\right]
$$

e

$$
\mathbf{f}^{e}=\underbrace{\int_{\Omega^{e}}\left(\mathbf{N}^{e}\right)^{T} f}_{f_{\Omega}}-\underbrace{\int_{\Gamma_{N}^{e}}\left(\mathbf{N}^{e}\right)^{T} g}_{f_{\Gamma_{N}}}=\left[\begin{array}{c}
\mathbf{f}_{1}^{e} \\
\mathbf{f}_{2}^{e} \\
\mathbf{f}_{3}^{e}
\end{array}\right]
$$

Como cada nó pode pertencer a mais de um elemento é necessário que as matrizes locais sejam reunidas num sistema global, somando as contribuições nodais em cada um dos elementos. Isto é feito somando-se as entradas associadas a cada um dos elementos, como observamos na Figura (2.5).

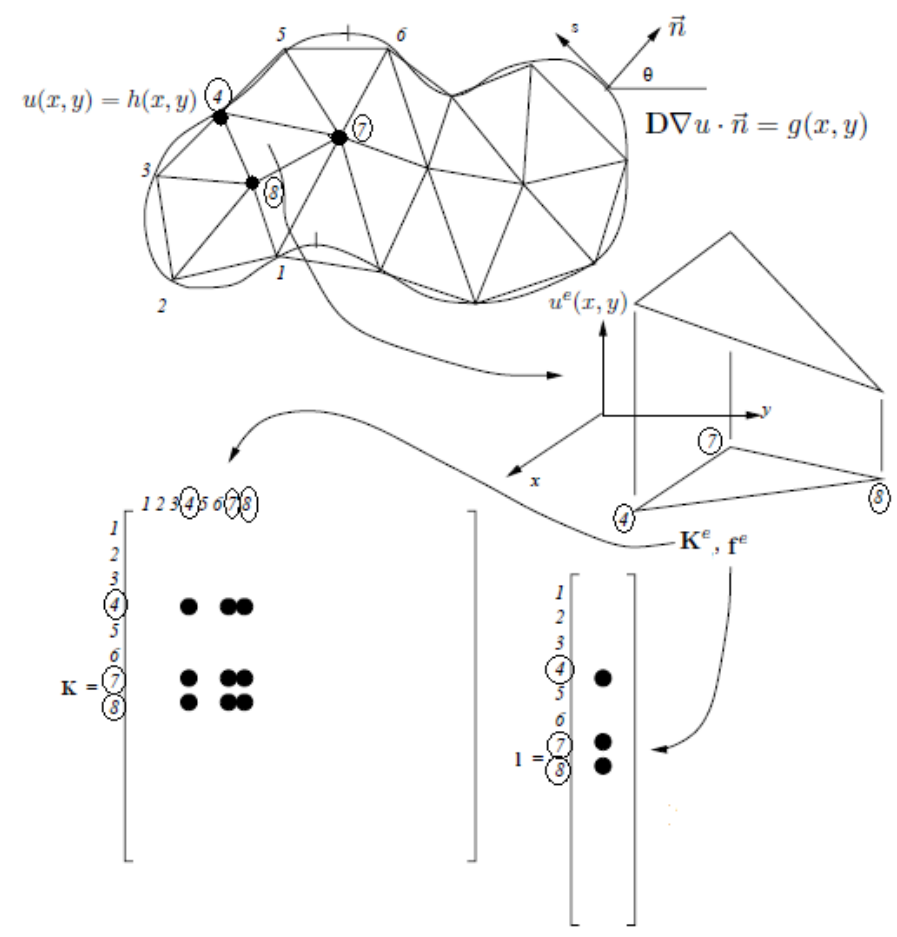

Figura 2.5: Montagem da matriz global de rigidez e do vetor global de forças. [22] 


\subsubsection{Estimativas na aproximação}

Sendo $u$ a solução do problema variacional e $u_{h}$ a solução do problema aproximado, o resultado a seguir fornece uma estimativa do erro cometido nesta aproximação. ${ }^{4}$

Teorema 2.4.12 (Teorema de Céa). Seja u solução de (2.10) e suponha que sejam válidas as condiçôes do teorema (2.1.15). Temos então que a solução $u_{h}$ do problema discreto satisfaz a seguinte relação:

$$
\left\|u-u_{h}\right\|_{V} \leq \frac{C}{\alpha} \min _{v \in V_{h}}\|u-v\|_{V}
$$

onde $C$ é a constante de continuidade e $\alpha$ a constante de coercividade do operador bilinear em $V$.

Demonstração. Utilizando a relação (2.16), a coercividade e a continuidade do operador $a(\cdot, \cdot)$, temos que para todo $v \in V_{h}$

$$
\begin{aligned}
\alpha\left\|u-u_{h}\right\|_{V}^{2} & \leq a\left(u-u_{h}, u-u_{h}\right) \quad \text { (Coercividade) } \\
& =a\left(u-u_{h}, u-v\right)+\underbrace{a\left(u-u_{h}, v-u_{h}\right)}_{=0} \quad\left(\text { como } v \in V_{h}, \text { então } v-u_{h} \in V_{h}\right) \\
& =a\left(u-u_{h}, u-v\right) \quad \\
& \leq C\left\|u-u_{h}\right\|_{V}\|u-v\|_{V} \quad \text { (Continuidade) }
\end{aligned}
$$

Logo, temos que

$$
\left\|u-u_{h}\right\|_{V} \leq \frac{C}{\alpha}\|u-v\|_{V}
$$

e, pela arbitrariedade de $v$ em $V_{h}$ concluimos a demonstração.

No caso em que o operador $a(\cdot, \cdot)$ é simétrico temos que o erro cometido na aproximação pelo método de Galerkin é ortogonal ao subespaço $V_{h}$ em relação ao produto interno induzido pelo operador $a$. Ou seja, a solução obtida pela método de Galerkin é a projeção ortogonal da solução exata no subespaço $V_{h}$. Neste caso, a desigualdade de Céa (2.27) pode ser substituida por

$$
\left\|u-u_{h}\right\|_{E}=\inf _{v \in V_{h}}\|u-v\|_{E}
$$

e a aproximação $u_{h}$ é a aproximação ótima de $u$ no subespaço $V_{h}$, com a norma $\|u\|_{E}=\sqrt{a(u, u)}$.

Utilizando a desigualdade de Céa, temos o seguinte resultado de convergência:

Corolário 2.4.13 (Convergência do Método de Galerkin). Suponha que sejam válidas as condições do teorema (2.4.12). Considere então $V_{h_{1}} \subset V_{h_{2}} \subset V_{h_{3}} \subset \cdots$ uma sequência de subespaços de $V$ com a propriedade:

$$
\overline{\bigcup_{i \geq 1} V_{h_{i}}}=V
$$

Então o Método de Galerkin converge

$$
\left\|u-u_{h_{i}}\right\|_{V} \rightarrow 0, \quad i \rightarrow \infty
$$

Demonstração. Pelo argumento de densidade (2.28), podemos encontrar uma sequência $v_{i} \in V_{h_{i}}$, $i \geq 1$ tal que

$$
\left\|u-v_{i}\right\|_{V} \rightarrow 0, \quad i \rightarrow \infty
$$

Aplicando (2.27) temos que

$$
\left\|u-u_{h_{i}}\right\|_{V} \leq \frac{C}{\alpha}\left\|u-v_{i}\right\|_{V}
$$

e, portanto, concluimos a convergência do método.

\footnotetext{
${ }^{4}$ Apesar de algumas referências apresentarem esse resultado como Lema de Céa preferimos apresentá-lo como um teorema pela sua importância no contexto deste trabalho.
} 


\subsection{FreeFem ++}

Nesta seção iremos realizar uma breve apresentação do software FreeFem ++ utilizado para obter as aproximações apresentadas neste trabalho, de modo que o leitor seja capaz de compreender os códigos utilizados nas simulações (que se encontram no Apêndice (B)). A referência [18] apresenta a descrição de todas as funcionalidades do programa de maneira muito mais completa, inclusive contendo diversos códigos modelos que serviram como base para os resultados deste trabalho.

O FreeFem ++ é um software livre utilizado para resolver EDPs utilizando o Método dos Elementos Finitos (FEM, em inglês) com o uso de diversos tipos de elementos e malhas para problemas em 2D ou em 3D.

Podemos destacar como principais características do programa a simplicidade do gerador de malhas incluso no programa, a facilidade na construção do espaço de funções (é feita com apenas um comando), a grande variedade de espaços de funções disponíveis (como polinômios lineares, quadráticos, Raviart-Thomas, etc) e a maneira eficiente e direta com que são feitas a implementação da formulação fraca associada ao MEF e a resolução do sistema linear associado (que permite utilizar diversos solvers: Gradientes Conjugados, GMRes, etc). Além disso, o programa dispõe de um visualizador gráfico bastante simples, que auxilia na visualização dos resultados obtidos.

A escolha do FreeFem ++ para a implementação das simulações contidas neste trabalho ocorreu devido a facilidade na construção e obtenção das aproximações por elementos finitos para os problemas aqui apresentados. No entanto, o programa mostrou-se capaz de analisar problemas bastante complexos.

\subsubsection{Como funciona}

Dado um domínio $\Omega \subset \mathbb{R}^{2}$ (ou $\mathbb{R}^{3}$ ) e uma EDP em sua formulação integral, o esquema geral para o funcionamento do programa utiliza os seguintes componentes:

1. Descrição da(s) fronteira(s) do domínio $\Omega$

O comando border Fronteira $(\mathrm{t}=\mathrm{a}, \mathrm{b})\{x=x(t), y=y(t)\}$ define a curva $(\mathrm{x}, \mathrm{y})$ parametrizada pelo parâmetro $t$ variando de $a$ até $b$, com $a, b \in \mathbb{R}$. Por exemplo, considerando $\Omega$ sendo o circulo unitário temos que a fronteira do circulo é obtida por

$$
\text { border } \mathrm{F}\left(\mathrm{t}=0,2^{*} \mathrm{pi}\right)\{x=\cos (t) ; y=\sin (t) ;\}
$$

2. Discretização do domínio $\Omega$ em um conjunto de elementos triangulares $\mathcal{T}$

O comando mesh cria uma variável que as informações de uma determinada discretização do domínio por elementos finitos. A construção da discretização é feita pelo comando buildmesh (Fronteira $(n)$ ), onde $n$ indica a quantidade de nós que estarão na fronteira. Por exemplo, para construirmos uma malha Th contendo 60 pontos na fronteira utilizamos o comando

$$
\text { mesh } \mathrm{Th}=\text { buildmesh }(\mathrm{F}(50)) \text {; }
$$

3. Geração do espaço de funções de forma definida sobre os elementos triangulares:

Para a geração do espaço de funções é utilizado o comando fespace(Th,Tipo), onde Th é a discretização do domínio em elementos triangulares e Tipo indica o tipo de elemento que será utilizado. Por exemplo, para aproximações lineares (polinômios de primeira ordem), utilizamos o comando

$$
\text { fespace } \mathrm{Vh}=(\mathrm{Th}, \mathrm{P} 1)
$$

4. Definição das funções do problema

No FreeFem ++ funções são definidas utilizando o comando func. Para definirmos a função $f(x, y)=x y$ utilizamos 
func $\mathrm{f}=\mathrm{x}^{*} \mathrm{y}$

5. Implementação da formulação variacional e dos respectivos valores de contorno Neste ponto serão necessárias três etapas:

- Integração numérica: A integração bidimensional sobre cada um dos elementos da discretização Th é feita utiliza-se o comando int2d(Th). As derivadas de $u$ em relação a $x$ e em relação a $y$ são descritas por $\mathrm{dx}(\mathrm{u})$ e $\mathrm{dx}(\mathrm{u})$, respectivamente.

- Atribuição das condições de contorno: As condições de contorno são impostas por meio do comando on(Fronteira, $\mathrm{u}=\ldots)$.

- Solução do sistema linear: É obtida por meio do comando solve(u,v).

Tomando como exemplo o problema de Poisson com $f(x, y)=x y$ e condições de contorno nulas, utilizaremos que a formulação fraca para este problema é dada por (3.3) e a resolução do problema é obtida com o comando

$$
\text { solve Poisson }(\mathrm{u}, \mathrm{v})=\operatorname{int} 2 \mathrm{~d}(\mathrm{Th}) \mathrm{dx}(\mathrm{u}) \mathrm{dx}(\mathrm{v})+\mathrm{dy}(\mathrm{u}) \mathrm{dy}(\mathrm{v})-\operatorname{int} 2 \mathrm{~d} f \mathrm{v}+\mathrm{on}(\mathrm{F}, \mathrm{u}=0) \text {; }
$$

6. Visualização dos resultados obtidos

As aproximações obtidas são visualizadas por meio do comando plot(Aprox, wait=1). O parâmtro wait $=1$ é utilizado para que a aproximação se mantenha na tela até que seja pressionada alguma tecla. O capítulo 7 da referência [18] apresenta em detalhes todos os parâmetros que podem ser utilizados nesta função (alguns deles facilitam bastante a geração das imagens).

\subsubsection{Outros comandos}

Nas simulações realizadas foram utilizados também os comandos auxiliares

- varf: Funciona como uma função global do programa

- intalledges(Th): Avalia um determinado operador em todas as arestas internas dos elementos da discretização Th.

- adaptmesh(Th,h,...): Dada uma malha inicial Th, este comando faz o refinamento adaptativo da malha de acordo com uma função $h$ previamente definida. Os parâmetros auxiliares são encontrados na seção 5.5 da referência [18], pág 115.

\subsection{Refinamento das malhas de estudo}

Nesta seção vamos analisar duas possíveis estratégias para o refinamento da malha utilizada na construção das aproximações por elementos finitos: as malhas obtidas por particionamento uniforme dos elementos (splitting) e as malhas refinadas por funções indicadoras de erro.

\subsubsection{Particionamento uniforme}

O refinamento uniforme (ou refinamento por splitting) é feito por meio da subdivisão de cada um dos elementos triangulares ${ }^{5}$ que compõem o domínio, de modo a gerar elementos menores com as mesmas propriedades geométricas do elemento original.

A principal vantagem deste refinamento é a simplicidade: cada triângulo pode ser dividido em outros 4 com a inserção dos novos nós nos pontos médios de cada aresta que compõem o elemento. Desta forma, todos os elementos serão admissíveis e as aproximações podem ser obtidas sobre a nova malha de elementos.

\footnotetext{
${ }^{5} \mathrm{~A}$ ideia é análoga para elementos quadriláteros ou, no caso no $\mathbb{R}^{3}$, prismas e tetrahedros.
} 


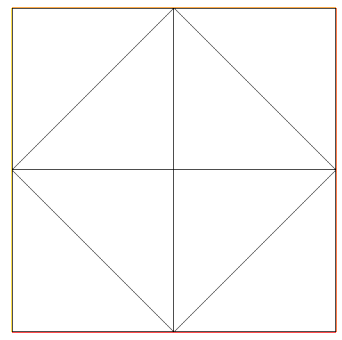

(a) Malha inicial

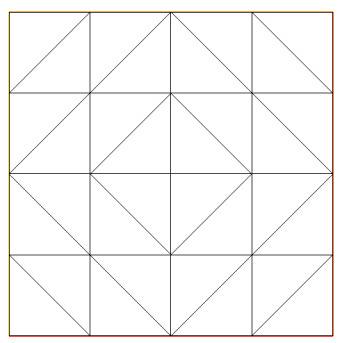

(b) Primeiro refinamento

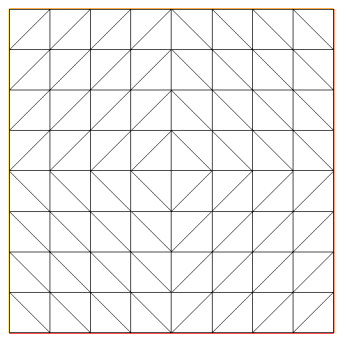

(c) Segundo refinamento

Figura 2.6: Aplicações do refinamento por splitting

A principal desvantagem deste esquema deve-se ao fato de que o refinamento uniforme insere novos nós em todo o domínio, inclusive em regiões onde o erro cometido na aproximação era suficientemente pequeno. Com isto, temos um aumento considerável no número de incógnitas do sistema linear a ser resolvido, de modo que o algoritmo irá utiizar um maior tempo computacional para obter as aproximações desejadas.

\subsubsection{Particionamento orientado por funções indicadoras}

Como alternativa ao particionamento uniforme, vamos construir uma estratégia de refinamento cujo objetivo é inserir novos nós somente nas regiões onde o erro cometido na aproximação foi considerado grande.

O primeiro desafio reside em como obter os erros cometidos nas aproximações, levando-se em conta que para problemas reais a solução exata do problema é desconhecida. A construção deste estimador será feita em detalhes mais adiante e, como exemplo, considere que utilizemos a malha descrita na Figura (2.2a) para a obtenção das aproximações pelo MEF. Utilizando os resultados obtidos, estimamos o erro cometido na aproximação conforme apresentado na Figura (2.2b).

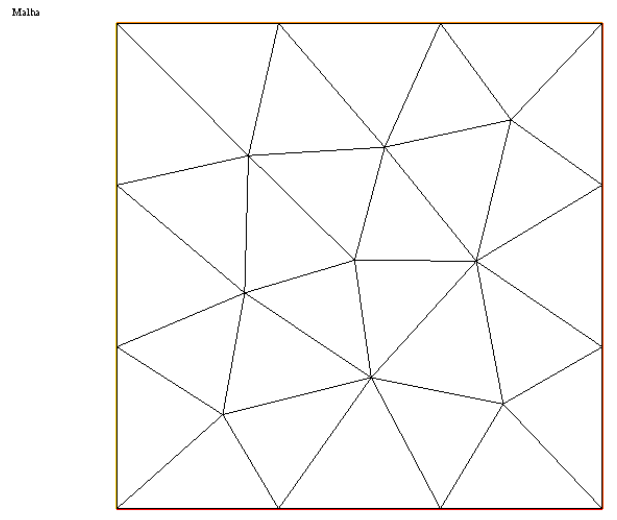

(a) Malha inicial
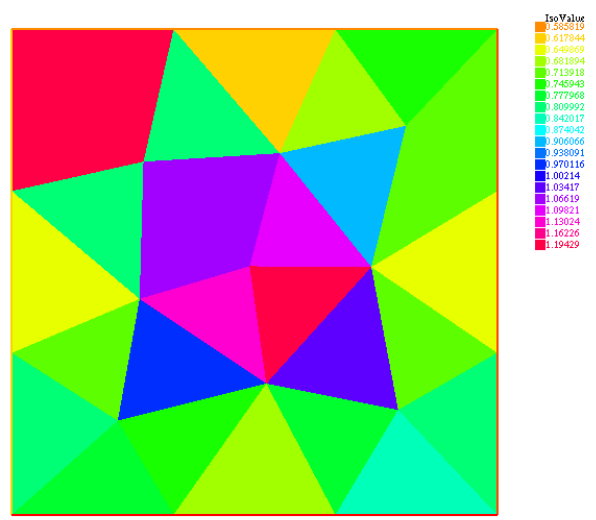

(b) Estimativa do erro

Figura 2.7: Malha inicial e estimativa do erro cometido. As cores mais escuras indicam um maior erro cometido nestas regióes.

Observamos que a estimativa de erro não é uniforme em todo o domínio: as regiões onde o indicador apresentou valores maiores (cores entre azul escuro e vermelho) estão concentradas no centro e na região superior esquerda enquanto que o restante do domínio apresentou valores menores na estimativa do erro cometido. Ao invés de refinarmos todo o domínio por igual vamos reduzir 
a área dos elementos conforme o valor obtido pelo estimador do erro: quanto maior a estimativa, maior a quantidade de partições a serem construidas na região em estudo.

Existem diversas técnicas de geração e refinamento de malha, cujos objetivos vão além do escopo deste trabalho. Em nossas simulações vamos considerar a estratégia sugerida em [18]: dado $x \in \Omega \mathrm{e}$ uma discretização $\mathcal{T}_{n}$, seja $\eta_{n}(x)$ é o valor do indicador para o erro no ponto $x \in \mathcal{T}_{n}, h_{n}$ o diâmetro do elemento ao qual o ponto $x$ esteja contido, $\eta^{\star}$ é o produto da média dos erros cometidos nos elementos por uma constante ${ }^{6}$ e $f_{n}=\min \left(3, \max \left(1 / 3, \eta_{n} / \eta_{n}^{\star}\right)\right)$. Nestas condições, definimos o diâmetro do elemento onde esteja o ponto $x$ na nova discretização $\mathcal{T}_{n+1}$, como

$$
h_{n+1}(x)=\frac{h_{n}(x)}{f_{n}\left(\eta_{K}(x)\right)}
$$

A função $f_{n}$ é utilizada para certificar-se de que a nova malha será de forma regular, evitando assim o surgimento de erros causados por problemas na geometria do domínio.

Utilizando a estratégia (2.30) na malha contida na Figura(2.2a), obtivemos a malha contida na Figura (2.3a).

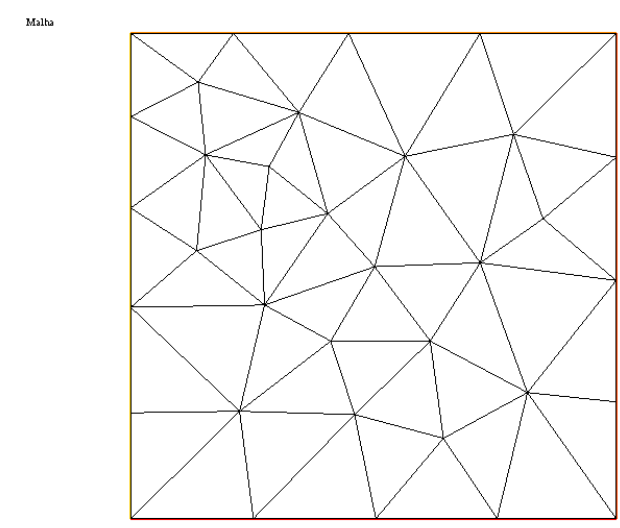

(a) Nova malha

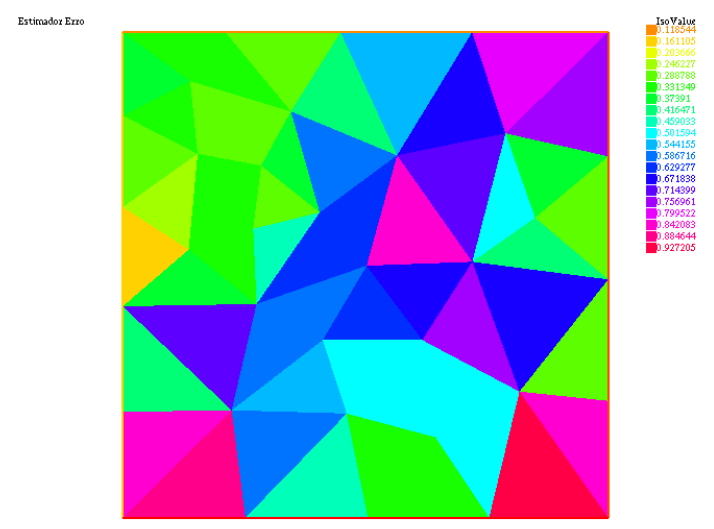

(b) Nova estimativa do erro

Figura 2.8: Malha obtida pelo refinamento indicado pelo estimador de erro

Conforme esperado, podemos observar que a nova discretização apresentou um número maior de elementos nas regiões onde o estimador apontou valores maiores para os erros cometidos na aproximação. A nova estimativa de erro, apresentada na Figura (2.3b), mostra como as regiões de maior erro variam entre as discretizações.

\footnotetext{
${ }^{6}$ Em geral, a constante utilizada é suficientemente próxima de 1.0 [18], pág 238.
} 


\section{Capítulo 3}

\section{Problema de Poisson}

Vamos introduzir o estudo da estimativa residual a partir do Problema de Poisson. Iniciamos o capítulo com a apresentação do problema, sua formulação fraca e a obtenção das aproximações pelo método de Ritz-Galerkin. Em seguida, construiremos a estimativa residual associada ao problema e apresentamos o resultado que relaciona a estimativa residual com o erro cometido pela aproximação. Por fim, vamos comparar as aproximações obtidas nas discretizações construidas por meio do refinamento uniforme e do refinamento adaptativo, ambos aplicados à um problema com solução conhecida e um problema que apresenta uma singularidade em seu contorno.

\subsection{Formulação fraca}

Considere o problema de Poisson

$$
\begin{cases}-\Delta u=f(x, y), & (x, y) \in \Omega \\ u(x, y)=0, & (x, y) \in \Gamma\end{cases}
$$

Vamos então introduzir a formulação fraca deste problema e discutir suas relações entre si.

Uma solução clássica de (3.1) é uma função $u \in \mathcal{C}^{2}(\Omega) \cap \mathcal{C}(\bar{\Omega})$ que satisfaz a equação diferencial para todo ponto no interior do domínio e satisfaz a condição de contorno para todo ponto na fronteira do domínio. Vamos supor que $f \in L^{2}(\Omega)$, apesar de que esta condição não é suficiente para garantir a existência de uma solução clássica para o problema.

Para obtermos a formulação fraca associada ao problema, suponha que exista uma solução $u \in \mathcal{C}^{2}(\Omega) \cap \mathcal{C}(\bar{\Omega})$ do problema (3.1). Multiplicamos a equação diferencial do problema por uma função $v \in \mathcal{C}_{0}^{\infty}(\Omega)$, a qual denominamos função de ponderação (ou função peso) e integramos o produto obtido sobre o domínio do problema, obtendo que

$$
-\int_{\Omega} v \Delta u=-\int_{\Omega} v \nabla \cdot(\nabla u)=\int_{\Omega} v f
$$

Utilizando integração por partes na integral do lado esquerdo da equação acima, como $v=0$ em $\Gamma$, temos que

$$
\int_{\Omega} \nabla u \cdot \nabla v=\int_{\Omega} v f
$$

Observe que a relação acima não utiliza o fato de que $u \in \mathcal{C}^{2}(\Omega) \cap \mathcal{C}(\bar{\Omega})$ mas sim que $u \in H_{0}^{1}(\Omega)$. Desta forma, o problema de valor de contorno (3.1) é equivalente à:

Definição 3.1.1 (Formulação fraca do problema de Poisson homogêneo). Obter $u \in H_{0}^{1}(\Omega)$ tal que $^{1}$ :

$$
\int_{\Omega} \nabla u \cdot \nabla v=\int_{\Omega} f v
$$

\footnotetext{
${ }^{1}$ Pelo Teorema $(2.1 .10)$, temos que $\mathcal{C}_{0}^{\infty}(\Omega)$ é denso em $H_{0}^{1}(\Omega)$ de modo que a relação 3.2 é valida para qualquer $v \in H_{0}^{1}$
} 
para toda função $v \in H_{0}^{1}(\Omega)$.

Observe que a condição $f \in L^{2}(\Omega)$ é mais forte do que o necessário neste caso: é suficiente que $f$ esteja no conjunto dual de $H_{0}^{1}(\Omega)$ dado que podemos interpretar a integral $\int_{\Omega} f(x) v(x) d x$ como o operador dual $\langle f, v\rangle$ entre $\left(H_{0}^{1}(\Omega)\right)^{\prime}$ e $H_{0}^{1}(\Omega)$.

Vamos mostrar agora que se $u$ é solução da formulação fraca (3.6), então $u$ é uma solução classica do problema (3.1). Supondo $f \in \mathcal{C}(\Omega)$ e que $u$ tenha a condição adicional de regularidade $u \in \mathcal{C}^{2}(\Omega) \cap \mathcal{C}(\bar{\Omega})$, temos que

$$
\int_{\Omega}(-\Delta u-f) v=0
$$

para toda $v \in \mathcal{C}_{0}^{\infty} \subset H_{0}^{1}(\Omega)$. Portanto, pelo Lema (2.1.5) temos que $-\Delta u=f$ em $\Omega$, ou seja, a equação diferencial (3.1) será satisfeita. Concluimos então que o problema de contorno (3.1) é formalmente equivalente a formulação fraca (3.3).

Considere $V=H_{0}^{1}(\Omega), a(\cdot, \cdot): V \times V \rightarrow \mathbb{R}$ a forma bilinear definida por

$$
a(u, v)=\int_{\Omega} \nabla u \cdot \nabla v
$$

e $l: V \rightarrow \mathbb{R}$ um funcional definido por

$$
l(v)=\int_{\Omega} f v
$$

Para a aplicação do Lema de Lax-Milgram é necessário que a forma bilinear seja contínua e coerciva, dado que a linearidade e a continuidade do funcional são imediatas. A continuidade da forma bilinear é obtida utilizando-se da desigualdade de Cauchy-Schwarz:

$$
\begin{aligned}
|a(u, v)| & =\left|\int_{\Omega} \nabla u \cdot \nabla v\right| \leq \int_{\Omega}|\nabla u \cdot \nabla v| \\
& \leq\|\nabla u\|_{L^{2}(\Omega)}|| \nabla v \|_{L^{2}(\Omega)} \text { (Cauchy-Schwarz) } \\
& \leq\|u\|_{H_{0}^{1}(\Omega)}\|v\|_{H_{0}^{1}(\Omega)}
\end{aligned}
$$

Por outro lado, a demonstração da coercividade é feita utilizando a desigualdade de Poincaré:

$$
\begin{aligned}
\|w\|_{H_{0}^{1}(\Omega)}^{2} & =\|w\|_{L^{2}(\Omega)}^{2}+\|\nabla w\|_{L^{2}(\Omega)}^{2} \\
& \leq(1+C)\|\nabla w\|_{L^{2}(\Omega)}^{2} \quad \text { (Desig. Poincaré) } \\
& =(1+C) a(w, w)
\end{aligned}
$$

Logo, a formulação variacional do problema (3.1) consiste em obter $u \in V$ tal que

$$
a(u, v)=l(v), \quad \forall v \in V
$$

e, pelo Lema de Lax-Milgram, temos que o problema possui uma única solução.

No caso mais geral, consideramos o problema onde são prescritas diferentes tipos de condições no contorno:

$$
\left\{\begin{array}{lc}
-\Delta u=f(x, y), & (x, y) \in \Omega \\
\nabla u \cdot \vec{n}=g, & (x, y) \in \Gamma_{N} \\
u=0, & (x, y) \in \Gamma_{D}
\end{array}\right.
$$

onde $\vec{n}$ é o vetor normal unitário exterior a $\Gamma_{N}$. Neste caso, da integração por partes, temos que

$$
\int_{\Omega} v \nabla \cdot(\nabla u)=\int_{\Gamma_{N}} g v-\int_{\Omega} \nabla u \cdot \nabla v
$$

e a formulação fraca do problema é dada pelo seguinte problema: 
Definição 3.1.2 (Formulação fraca do problema de Poisson com condições mistas.). Obter $u \in$ $H_{D}^{1}(\Omega)$ tal que:

$$
\int_{\Omega} \nabla u \cdot \nabla v=\int_{\Omega} f v+\int_{\Gamma_{N}} g v
$$

para toda função $v \in H_{D}^{1}(\Omega)$.

Assim, considerando $V=H_{D}^{1}(\Omega)$, o problema é escrito na forma (2.10) com

$$
a(u, v)=\int_{\Omega} \nabla u \cdot \nabla v
$$

$\mathrm{e}$

$$
l(v)=\int_{\Omega} f v+\int_{\Gamma} g v
$$

onde, sob as condições de que $f \in L^{2}(\Omega)$ e $g \in L^{2}\left(\Gamma_{N}\right)$, temos que

$$
\begin{aligned}
l\left(v_{1}+v_{2}\right) & =\int_{\Omega} f\left(v_{1}+v_{2}\right)+\int_{\Gamma_{N}} g\left(v_{1}+v_{2}\right) \\
& =\int_{\Omega} f v_{1}+\int_{\Gamma_{N}} g v_{1}+\int_{\Omega} f v_{2}+\int_{\Gamma_{N}} g v_{2} \\
& =l\left(v_{1}\right)+l\left(v_{2}\right) \text { (Linearidade) }
\end{aligned}
$$

$\mathrm{e}$

$$
\begin{aligned}
|l(v)| & =\left|\int_{\Omega} f v+\int_{\Gamma_{N}} g v\right| \leq \int_{\Omega}|f v|+\int_{\Gamma_{N}}|g v| \\
& \leq\|f\|_{L^{2}} \cdot\|v\|_{L^{2}}+\|g\|_{L^{2}} \cdot\|v\|_{L^{2}} \\
& \leq\left(\|f\|_{L^{2}}+\|g\|_{L^{2}}\right)\|v\|_{L^{2}} \\
& \leq C\|v\|_{L^{2}} \leq C\|v\|_{H_{D}^{1}} \text { (Continuidade) }
\end{aligned}
$$

podemos aplicar novamente o Lema de Lax-Milgram e concluir a existência e unicidade de solução do problema.

Dado que a forma bilinear associada ao problema satisfaz as hipóteses para existência e unicidade de solução do problema contínuo, vamos aplicar o Método de Ritz-Galerkin para obter uma solução aproximada para o problema. Considere $\mathcal{T}$ uma partição admissível do domínio contendo um conjunto $N_{\mathcal{T}}$ de vértices dos elementos e um conjunto de funções de forma definidas sobre as variáveis nodais.

Nestas condições, a formulação fraca do problema definido sobre esta partição é descrita como:

Definição 3.1.3 (Formulação Fraca para a partição $\mathcal{T}$ ). Obter $u_{\tau} \in S_{D}^{1,0}(\mathcal{T})$ é tal que:

$$
\int_{\Omega} \nabla u_{\tau} \cdot \nabla v_{\tau}=\int_{\Omega} f v_{\tau}+\int_{\Gamma_{N}} g v_{\tau}
$$

para toda função $v_{\tau} \in S_{D}^{1,0}(\mathcal{T})$.

\subsection{Estimativa residual}

Dado que a forma bilinear $a(\cdot, \cdot)$ define um produto interno e uma norma em $H_{D}^{1}(\Omega)$, nosso objetivo será construir um estimador de erro $\eta$ para a forma bilinear tal que

$$
a\left(u-u_{\tau}, u-u_{\tau}\right) \leq \eta\left\|u-u_{\tau}\right\|_{1}
$$


Utilizando a coercividade do operador $a(\cdot, \cdot)$ obtemos que

$$
\left\|u-u_{\tau}\right\|_{1}^{2} \leq C a\left(u-u_{\tau}, u-u_{\tau}\right) \leq C \eta\left\|u-u_{\tau}\right\|_{1}
$$

e, portanto

$$
\left\|u-u_{\tau}\right\|_{1} \leq C \eta
$$

onde $C$ é uma constante que depende apenas da partição $\mathcal{T}$.

Sendo $u$ e $u_{\tau}$ as soluções dos problemas (3.6) e (3.7), respectivamente, observe que $u$ e $u_{\tau}$ satisfazem a seguinte relação

$$
a\left(u-u_{\tau}, v\right)=\int_{\Omega} \nabla\left(u-u_{\tau}\right) \cdot \nabla v=\int_{\Omega} f v+\int_{\Gamma_{N}} g v-\int_{\Omega} \nabla u_{\tau} \cdot \nabla v
$$

para toda $v \in H_{D}^{1}(\Omega)$. Desta forma, podemos definir o resíduo obtido de $u_{\tau}$ como

$$
\mathcal{R}\left(u_{\tau}, v\right)=\int_{\Omega} f v+\int_{\Gamma_{N}} g v-\int_{\Omega} \nabla u_{\tau} \cdot \nabla v
$$

O resultado a seguir relaciona o resíduo com o erro cometido pela aproximação, utilizando a norma de $H_{D}^{1}(\Omega)$ :

Teorema 3.2.1 (Relação entre o resíduo e o erro). O erro cometido na aproximação $u_{\tau}$ é limitado pela norma do resíduo no espaço dual de $H_{D}^{1}(\Omega)$.

Demonstração. Inicialmente, observe que o resíduo $\mathcal{R}\left(u_{\tau}, v\right)$ é um elemento contido no espaço dual do conjunto $H_{D}^{1}(\Omega)$. Além disso, utilizando as desigualdades de Cauchy-Schwarz e de Poincaré, temos que

$$
\frac{1}{\sqrt{1+c^{2}}}\|u\|_{1} \leq \sup _{\substack{w \in H_{D}^{1}(\Omega) \\\|w\|_{1}=1}} \int_{\Omega} \nabla u \cdot \nabla w \leq\|u\|_{1}
$$

para toda função $v \in H_{D}^{1}(\Omega)$. Isto implica que a forma bilinear

$$
H_{D}^{1}(\Omega) \ni u, w \mapsto \int_{\Omega} \nabla u \cdot \nabla w
$$

define um isomorfismo de $H_{D}^{1}(\Omega)$ no seu espaço dual. As constantes multiplicativas dos termos a direita e a esquera da desigualdade são relacionadas pela norma do isomorfismo e da sua inversa.

Da definição do resíduo e da desigualdade anterior, segue que

$$
\sup _{\substack{w \in H_{D}^{1}(\Omega) \\\|w\|_{1}=1}} \mathcal{R}\left(u_{\tau}, w\right) \leq\left\|u-u_{\tau}\right\|_{1} \leq \sqrt{1+c^{2}} \sup _{\substack{w \in H_{D}^{1}(\Omega) \\\|w\|_{1}=1}} \mathcal{R}\left(u_{\tau}, w\right)
$$

Como o termo

$$
\sup _{\substack{w \in H_{D}^{1}(\Omega) \\\|w\|_{1}=1}}\left\{\int_{\Omega} f w+\int_{\Gamma_{N}} g w-\int_{\Omega} \nabla u_{\tau} \cdot \nabla w\right\}
$$

é equivalente a norma do resíduo no espaço dual, concluimos o resultado.

Nosso objetivo será construir uma estimativa para a norma do resíduo no espaço dual utilizando os dados da partição $\mathcal{T}$, as aproximações $u_{\tau}$ obtidas e as funções $f$ e $g$. 
Dado que a aproximação obtida é de classe $\mathcal{C}^{2}$ no interior dos elementos é possível aplicar o operador laplaciano sobre a aproximação $u_{\tau}$ e aplicar a integração por partes no último termo a direita do resíduo, de forma que

$$
\begin{aligned}
\int_{\Omega} \nabla u_{\tau} \cdot \nabla v & =\sum_{K \in \mathcal{T}} \int_{K} \nabla u_{\tau} \cdot \nabla v \\
& =\sum_{K \in \mathcal{T}}\left(\int_{K} \Delta u_{\tau} v-\int_{\partial K} \vec{n}_{K} \cdot \nabla u_{\tau} v\right)
\end{aligned}
$$

Substituindo no resíduo, obtemos que

$$
\mathcal{R}\left(u_{\tau}, v\right)=\sum_{K \in \mathcal{T}} \int_{K}\left(f+\Delta u_{\tau}\right) v+\sum_{E \in \mathcal{E}_{\tau, \Gamma_{N}}} \int_{E}\left(g-\vec{n}_{E} \cdot \nabla u_{\tau}\right) v-\sum_{E \in \mathcal{E}_{\tau, \Omega}} \int_{E} \mathbb{J}\left(\vec{n}_{E} \cdot \nabla u_{\tau}\right) v
$$

onde $\mathbb{J}$ é a função de salto descrita em (2.20). Definimos o resíduo interior e o resíduo do bordo associado a cada elemento $K \in \mathcal{T}$ como

$$
R_{K}\left(u_{\tau}\right)=f+\Delta u_{\tau}
$$

$\mathrm{e}$

$$
R_{E}\left(u_{\tau}\right)=\left\{\begin{array}{lr}
-\mathbb{J}_{E}\left(\vec{n}_{E} \cdot \nabla u_{\tau}\right), & \text { se } E \in \mathcal{E}_{\tau, \Omega} \\
g-\vec{n}_{E} \cdot \nabla u_{\tau}, & \text { se } E \in \mathcal{E}_{\tau, \Gamma_{N}} \\
0, & \text { se } E \in \mathcal{E}_{\tau, \Gamma_{D}}
\end{array}\right.
$$

respectivamente. Desta forma, temos a chamada representação $L^{2}$ do resíduo:

$$
\mathcal{R}\left(u_{\tau}, v\right)=\sum_{K \in \mathcal{T}} \int_{K} R_{K}\left(u_{\tau}\right) v+\sum_{E \in \mathcal{E}_{\tau}} \int_{E} R_{E}\left(u_{\tau}\right) v
$$

Pela definição do resíduo e pela ortogonalidade de Galerkin (2.16) temos que

$$
\mathcal{R}\left(u_{\tau}, v_{\tau}\right)=\underbrace{a\left(u-u_{\tau}, v_{\tau}\right)}_{=0}=0
$$

de modo que podemos escrever

$$
\mathcal{R}\left(u_{\tau}, v\right)=\mathcal{R}\left(u_{\tau}, v\right)-\underbrace{\mathcal{R}\left(u_{\tau}, v_{\tau}\right)}_{=0}=\sum_{K \in \mathcal{T}} \int_{K} R_{K}\left(u_{\tau}\right)\left(v-v_{\tau}\right)+\sum_{E \in \mathcal{E}_{\tau}} \int_{E} R_{E}\left(u_{\tau}\right)\left(v-v_{\tau}\right)
$$

Fixada uma função $v \in H_{D}^{1}(\Omega)$, considere $v_{\tau}=I_{\tau} v$ a projeção de $v$ pelo operador de quasiinterpolação (2.18). Da desigualdade de Cauchy-Schwarz para integrais e das estimativas do operador (2.19), temos que

$$
\begin{aligned}
\mathcal{R}\left(u_{\tau}, v\right) & =\sum_{K \in \mathcal{T}} \int_{K} R_{K}\left(u_{\tau}\right)\left(v-I_{\tau} v\right)+\sum_{E \in \mathcal{E}_{\tau}} \int_{E} R_{E}\left(u_{\tau}\right)\left(v-I_{\tau} v\right) \\
& \leq \sum_{K \in \mathcal{T}}\left\|R_{K}\left(u_{\tau}\right)\right\|_{K} \underbrace{\left\|v-I_{\tau} v\right\|_{K}}_{\leq \alpha h_{K}\|v\|_{H^{1}\left(\tilde{\omega}_{K}\right)}}+\sum_{E \in \mathcal{E}_{\tau}}\left\|R_{E}\left(u_{\tau}\right)\right\|_{E} \underbrace{\left\|v-I_{\tau} v\right\|_{E}}_{\beta \sqrt{h_{E}}\|v\|_{H^{1}\left(\tilde{\omega}_{E}\right)}} \\
& \leq \sum_{K \in \mathcal{T}}\left\|R_{K}\left(u_{\tau}\right)\right\|_{K} \cdot \alpha h_{K}\|v\|_{1, \tilde{v}_{K}}+\sum_{E \in \mathcal{E}_{\tau}}\left\|R_{E}\left(u_{\tau}\right)\right\|_{E} \cdot \beta \sqrt{h_{E}}\|v\|_{1, \tilde{v}_{E}}
\end{aligned}
$$


Da desigualdade de Cauchy-Schwarz para as somas, temos que

$$
\begin{aligned}
\mathcal{R}\left(u_{\tau}, v\right) \leq \max _{\alpha, \beta}\left\{\sum_{K \in \mathcal{T}} h_{K}^{2}\left\|R_{K}\left(u_{\tau}\right)\right\|_{K}^{2}+\sum_{E \in \mathcal{E}_{\tau}} h_{E}\left\|R_{E}\left(u_{\tau}\right)\right\|_{E}^{2}\right\}^{\frac{1}{2}} . \\
\cdot\left\{\sum_{K \in \mathcal{T}}\|v\|_{1, \tilde{v}_{K}}^{2}+\sum_{E \in \mathcal{E}_{\tau}}\|v\|_{1, \tilde{v}_{E}}^{2}\right\}^{\frac{1}{2}}
\end{aligned}
$$

Da regularidade da malha $\mathcal{T}$, temos que

$$
\left\{\sum_{K \in \mathcal{T}}\|v\|_{1, \tilde{v}_{K}}^{2}+\sum_{E \in \mathcal{E}_{\tau}}\|v\|_{1, \tilde{v}_{E}}^{2}\right\}^{\frac{1}{2}} \leq c\|v\|_{1}
$$

onde $c$ é uma constante que depende apenas da malha utilizada. Da coercividade da forma bilinear, concluimos que

$$
\left\|u-u_{\tau}\right\|_{1} \leq C\left(\sum_{K \in \mathcal{T}} h_{K}^{2}\left\|R_{K}\left(u_{\tau}\right)\right\|_{K}^{2}+\sum_{E \in \mathcal{E}_{\tau}} h_{E}\left\|R_{E}\left(u_{\tau}\right)\right\|_{E}^{2}\right)^{\frac{1}{2}}
$$

onde $C$ é uma constante que depende somente da malha em estudo. Logo, provamos o seguinte teorema:

Teorema 3.2.2 (Estimador de Erro Residual a Posteriori). Sejam $u \in H_{D}^{1}(\Omega)$ e $u_{\tau} \in S_{D}^{1,0}(\mathcal{T})$ as soluções dos problemas (3.6) e (3.7), respectivamente. Para cada elemento $K \in \mathcal{T}$, definimos o estimador de erro residual a posteriori $\eta_{K}$ como:

$$
\begin{gathered}
\eta_{K}^{2}=h_{K}^{2}\left\|f+\Delta u_{\tau}\right\|_{K}^{2}+\frac{1}{2} \sum_{E \in \mathcal{E}_{K} \cap \mathcal{E}_{\tau, \Omega}} h_{E}\left\|\mathbb{J}\left(\vec{n}_{E} \cdot \nabla u_{\tau}\right)\right\|_{E}^{2} \\
+\sum_{E \in \mathcal{E}_{K} \cap \mathcal{E}_{\tau, \Gamma_{N}}} h_{E}\left\|g-\vec{n}_{E} \cdot \nabla u_{\tau}\right\|_{E}^{2}
\end{gathered}
$$

Sob estas condiçôes, temos que

$$
\left\|u-u_{\tau}\right\|_{1} \leq C\left(\sum_{K \in \mathcal{T}} \eta_{K}^{2}\right)^{\frac{1}{2}}
$$

onde $C$ é uma constante que depende apenas da malha utilizada.

O primeiro termo do estimador $\eta_{K}$ está relacionado ao resíduo da aproximação $u_{\tau}$ com respeito a EDP em sua formulação forte. Já os outros termos estão associados as condições de contorno do problema e são particularmente importantes devido ao fato que, se $u_{\tau} \notin H^{2}(\Omega)$, então $u_{\tau}$ não necessariamente satisfaz exatamente a condição de Neumann na fronteira. Logo, para aproximações de baixa ordem os termos associados as fronteiras são de fundamental importância para o estimador residual. 
A implementação computacional do estimador residual descrito no Teorema(3.2.2) encontra-se descrita no Algoritmo 1.

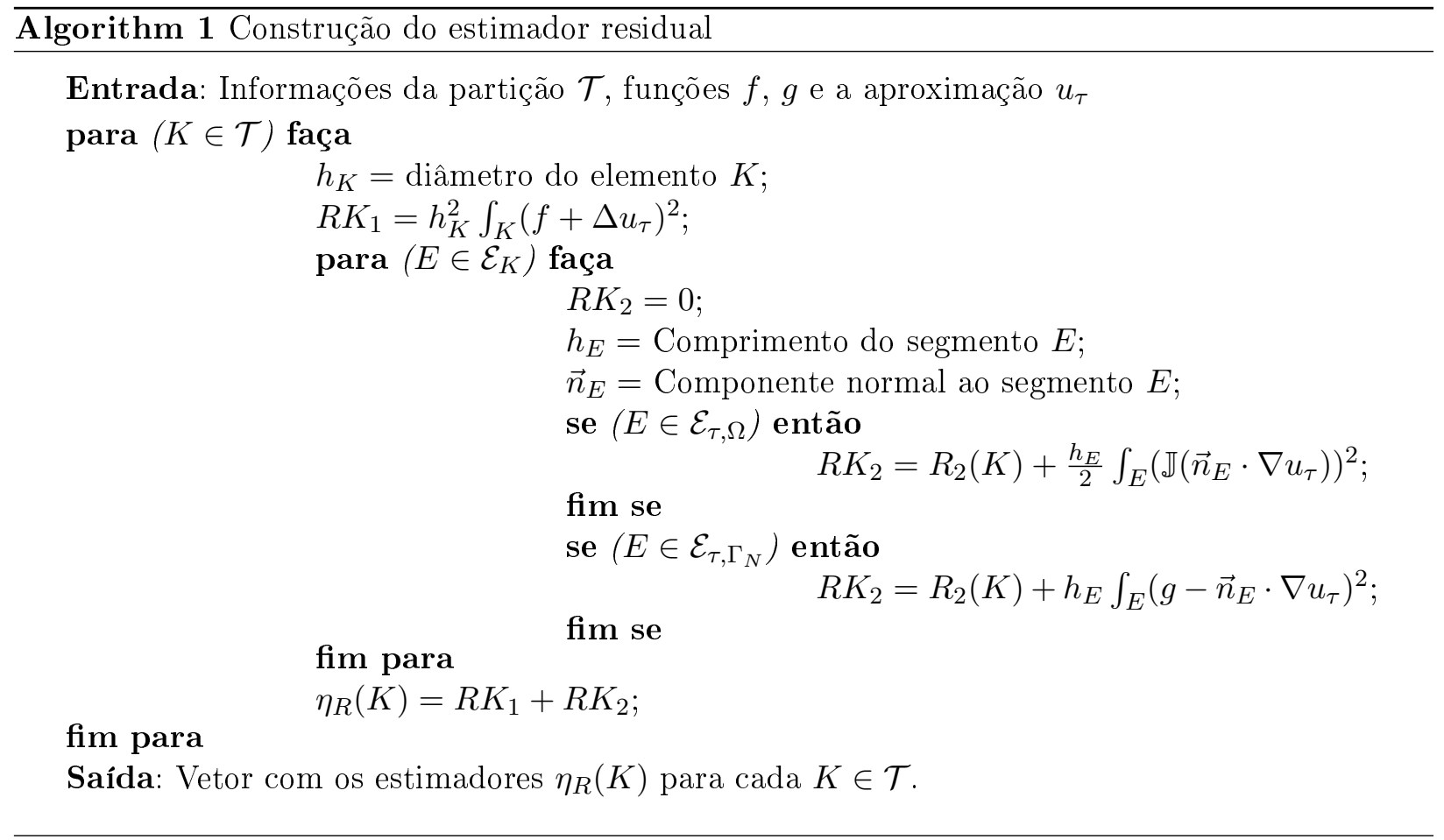

\subsection{Simulações Numéricas}

Para analisarmos os erros obtidos pelo estimador residual e as vantagens da aplicação do refinamento adaptativo das malhas, consideramos as seguintes quantidades:

Quantidade de nós da malha

Quantidade de elementos triangulares

Erro calculado pela norma L2

Erro obtido pelo estimador residual

$\varepsilon(\%)$ : Erro relativo estimado, calculado por $\frac{\eta}{\left\|u_{\tau}\right\|_{1}}$

Tempo computacional, calculado em $m s$.

Nossos objetivos serão comparar as soluções obtidas com os refinamentos uniforme e adaptativo, além de analisar o comportamento das aproximações obtidas e os erros cometidos. As figuras utilizadas neste capítulo encontram-se em escala reduzida para facilitar a visualização e a comparação dos resultados e apresentam em suas legendas o intervalo de variação dos resultados. As figuras encontram-se em seus tamanhos originais no Apêndice $(C)$.

\subsubsection{Problema com solução conhecida}

Vamos iniciar nossas simulações analisando os resultados obtidos na solução numérica do seguinte problema de Poisson:

$$
\begin{cases}-\Delta u=2 \pi^{2} \sin (\pi x) \sin (\pi y), & (x, y) \in \Omega \\ u(x, y)=0, & (x, y) \in \Gamma\end{cases}
$$

onde $\Omega$ é o quadrado unitário. A solução deste problema é dada por $u(x, y)=\sin (\pi x) \sin (\pi y)$. 


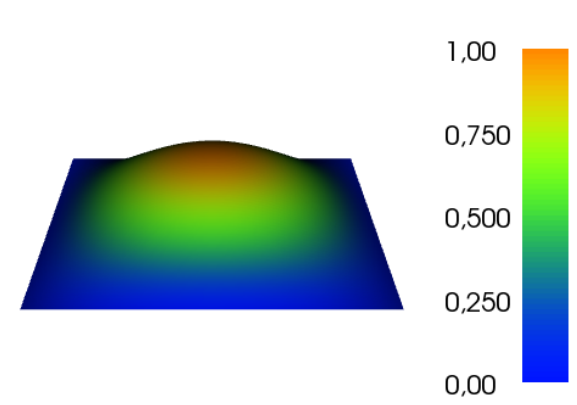

(a)

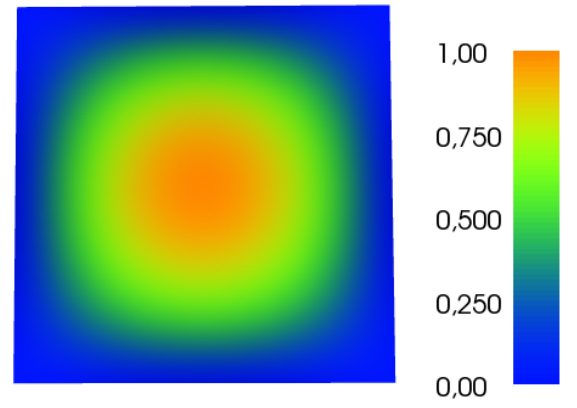

(b)

Figura 3.1: Solução exata do problema (3.16).

Utilizando elementos triangulares e funções de forma lineares sobre cada um dos elementos, obtivemos as aproximações e os estimadores residuais contidos nas Figuras (3.2(a)) e (3.2(b)).
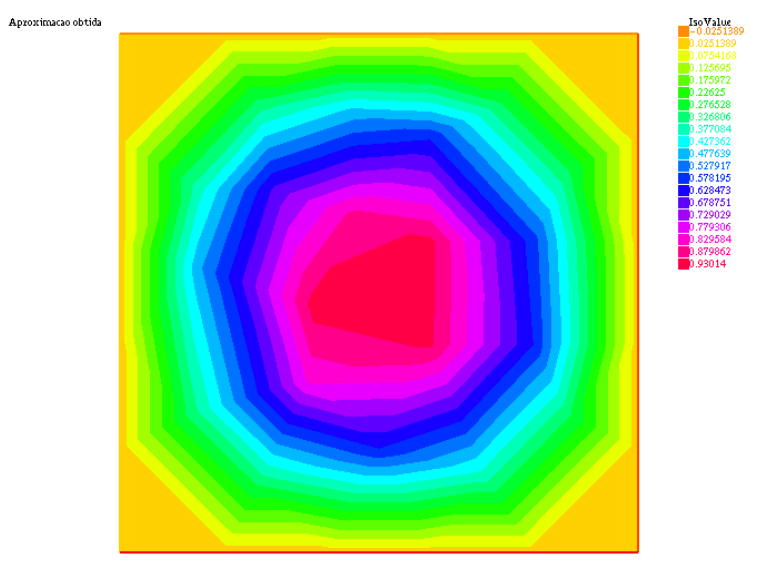

(a) Aproximação obtida $=\{0.0251389: 0.93014\}$
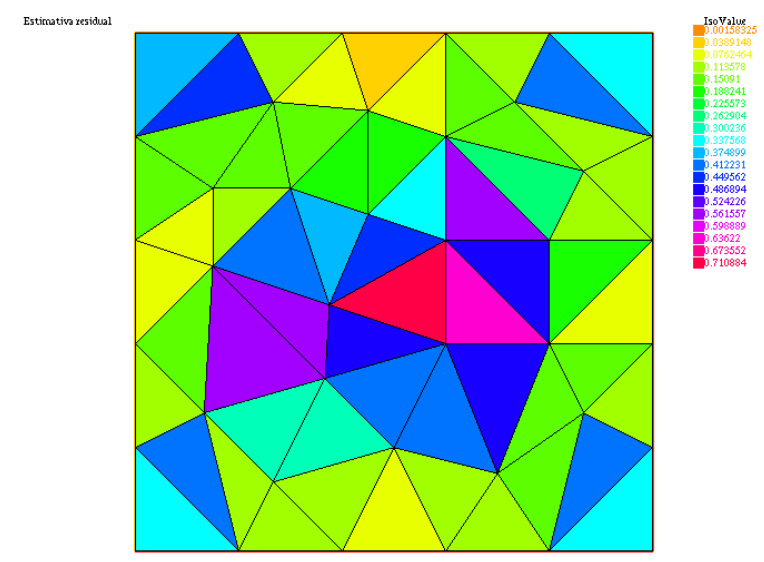

(b) Estimativa residual $=\{0.0158325: 0.710884\}$

Figura 3.2: Resultados obtidos sobre os elementos triangulares. 
É possível observar que existe uma maior variação na região central e ao redor dos pontos $(0,0),(1,0),(1,1)$ e $(0,1)$. Nas extremidades do domínio, o erro observado deve-se ao fato de que os três pontos dos elementos estão no bordo e, como a condição de contorno é nula, a aproximação obtida é nula em todo ponto contido nestes elementos. Como a solução exata é suave e apresenta o seu ponto de máximo no centro do quadrado, dado que o ponto de máximo não é um dos nós da partição e a aproximação gerada consiste de planos triangulares entre três nós contidos no domínio, temos que o ponto de máximo do domínio não faz parte dos valores nodais e é obtido por aproximação, acarretando assim em um maior valor de erro no elemento onde o máximo da função esta contido.

Para aumentar a precisão das aproximações realizamos uma iteração de refinamento uniforme, particionando cada um dos elementos triangulares em quatro novos elementos. A aproximação obtida e o estimador residual obtido encontram-se na Figura (3.3):

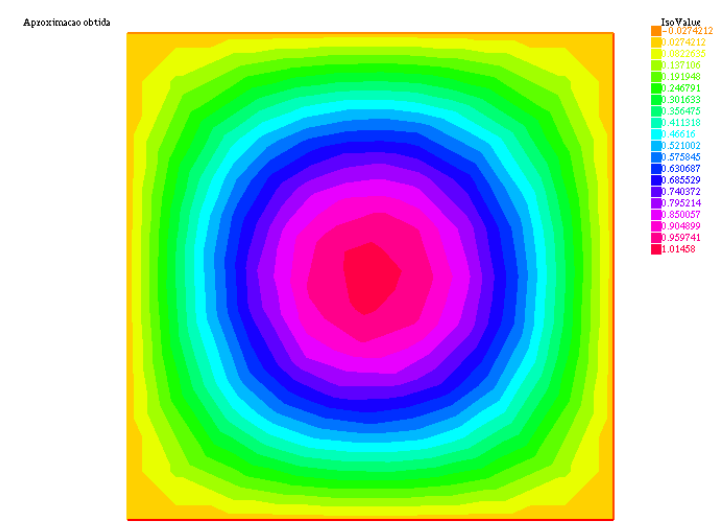

(a) Aproximação obtida $=\{0.0274212: 1.01458\}$

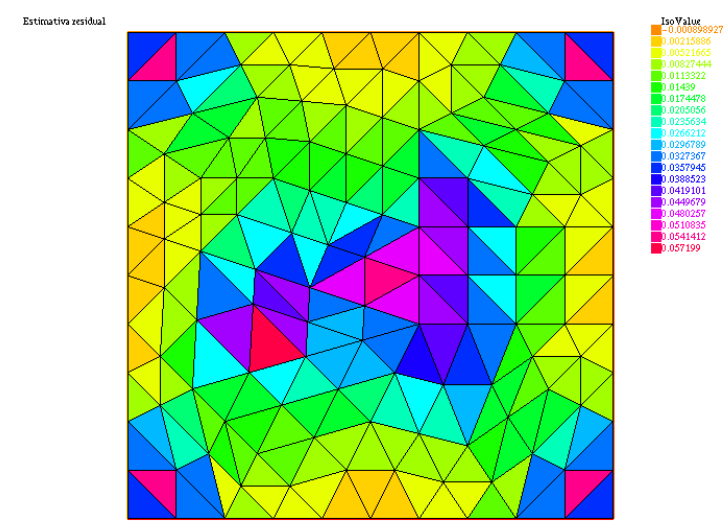

(b) Estimativa residual $=\{0.000898927: 0.057199\}$

Figura 3.3: Resultados obtidos na primeira iteração do refinamento uniforme.

Utilizando a mesma malha inicial, realizamos uma iteração do refinamento adaptativo, obtendo a aproximação e o estimador contidos na Figura (3.4):

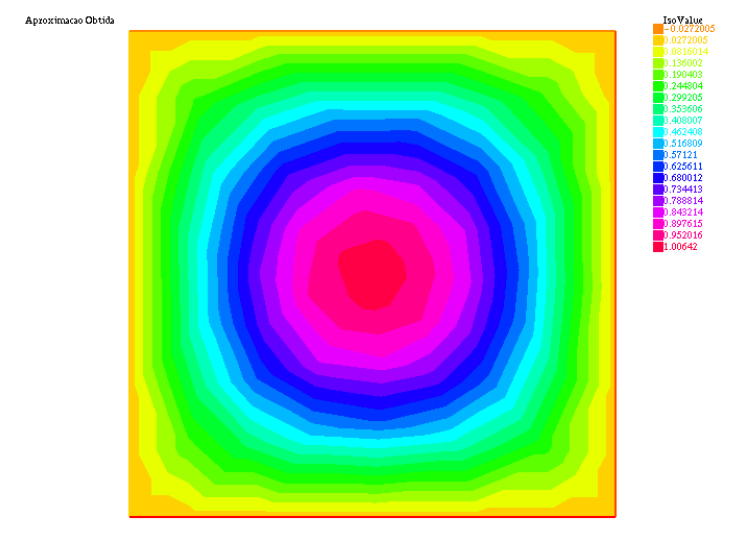

(a) Aproximação obtida $=\{0.0272005: 1.00642\}$

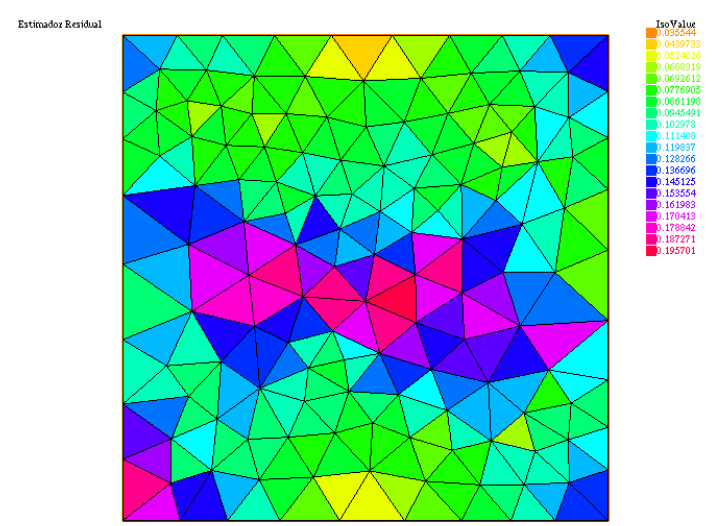

(b) Estimativa residual $=\{0.035544: 0.195701\}$

Figura 3.4: Resultados obtidos na primeira iteração do refinamento adaptativo. 


\begin{tabular}{|l|c|c|}
\hline & Uniforme & Adaptativo \\
\hline Quantidade de Nós & 137 & 73 \\
\hline Quantidade de Triângulos & 232 & 120 \\
\hline Erro na norma L2 & 0.01143 & 0.01646 \\
\hline Erro Residual & 1.98439 & 2.40086 \\
\hline$\varepsilon(\%)$ & 0.88055 & 1.07 \\
\hline Tempo computacional $(\mathrm{ms})$ & $<<1$ & $<<1$ \\
\hline
\end{tabular}

Tabela 3.1: Comparação entre o refinamento uniforme e o refinamento adaptativo.

A comparação entre a primeira iteração de ambos os refinamentos mostrou que o refinamento adaptativo foi capaz de obter uma aproximação com o erro na norma L2 da mesma ordem de grandeza $\left(10^{-2}\right)$ utilizando uma quantidade de nós muito menor do que aquela utilizada no refinamento uniforme (redução de aproximadamente $46 \%$ do número de nós).

Para reduzirmos ainda mais o erro cometido realizamos mais três iterações de refinamento, obtendo as aproximações e os estimadores residuais contidos nas Figuras (3.5) e (3.6).

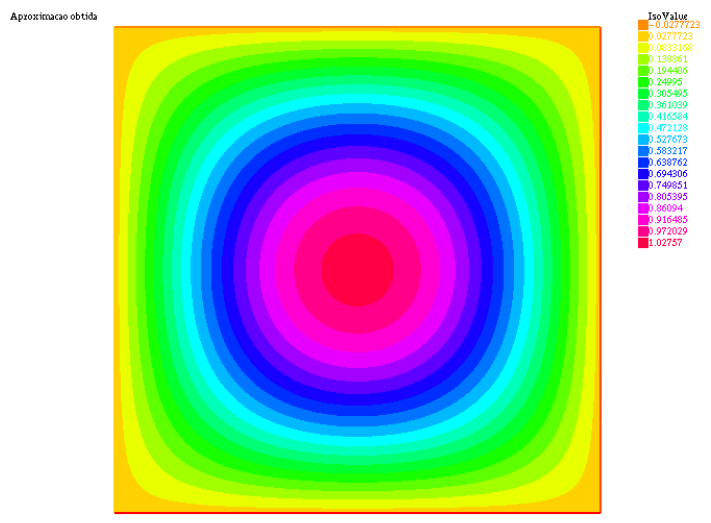

(a) Aproximação obtida $=\{0.0277723: 1.02757\}$

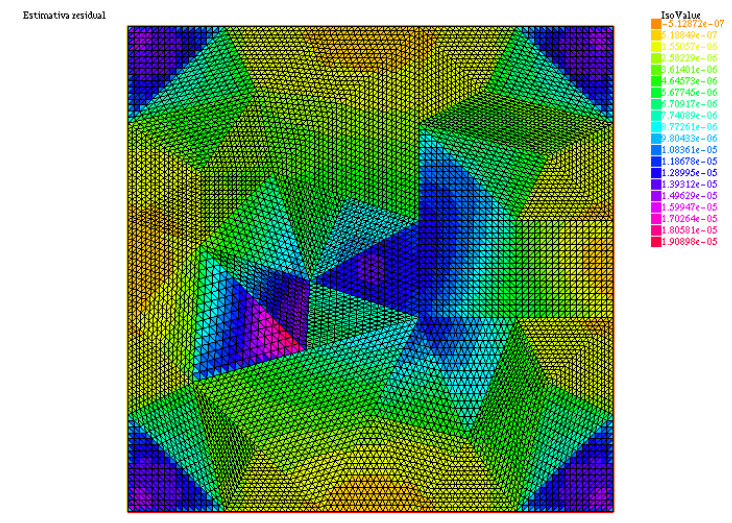

(b) Estimativa residual $=\{5.12872 \mathrm{e}-07: 1.90898 \mathrm{e}-05\}$

Figura 3.5: Resultados obtidos na quarta iteração do refinamento uniforme.

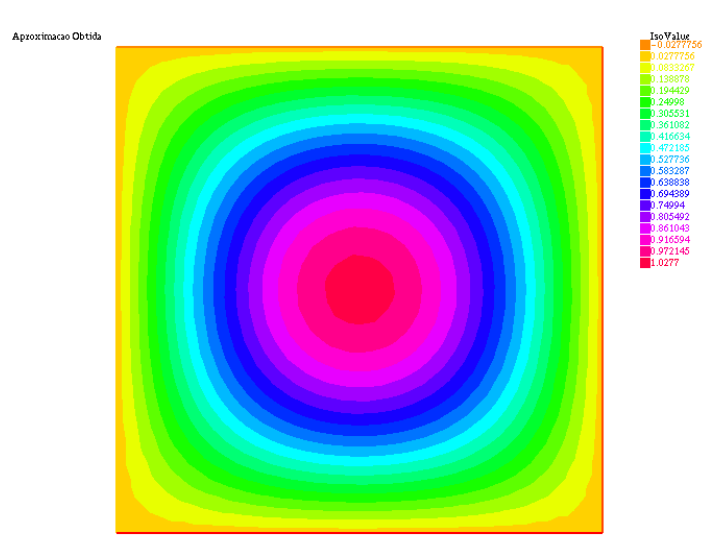

(a) Aproximação obtida $=\{-0.0277756: 1.0277\}$

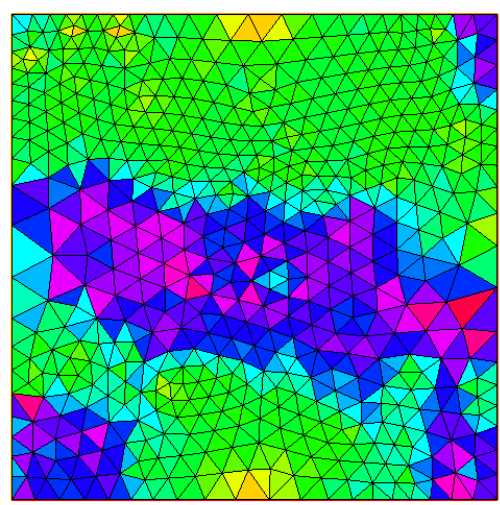

(b) Estimativa residual $=\{0.006453: 0.0491282\}$

Figura 3.6: Resultados obtidos na quarta iteração do refinamento adaptativo. 


\begin{tabular}{|l|c|c|}
\hline & Uniforme & Adaptativo \\
\hline Quantidade de Nós & 7585 & 573 \\
\hline Quantidade de Triângulos & 14848 & 1062 \\
\hline Erro na norma L2 & 0.000182 & 0.001692 \\
\hline Erro Residual & 0.259999 & 0.817044 \\
\hline$\varepsilon(\%)$ & 0.114203 & 0.359378 \\
\hline Tempo computacional $(m s)$ & 210 & 10 \\
\hline
\end{tabular}

Tabela 3.2: Comparação entre o refinamento uniforme e o refinamento adaptativo na quarta iteração de refinamento.

Comparando os estimadores residuais obtidos no refinamento uniforme (Figuras (3.2(b)), (3.4(b)) e (3.6(b)), observamos que as regiões onde o estimador indica valores maiores para o erro não variam entre as iterações de refinamento, ou seja, as regiões onde o estimador indica um valor maior de erro permanecem constantes mesmo após o refinamento. Por outro lado, o erro residual apresentado na malha gerada pelo refinamento adaptativo apresenta uma variação das regiões onde o erro é maior, como podemos observar na Figura (3.7).

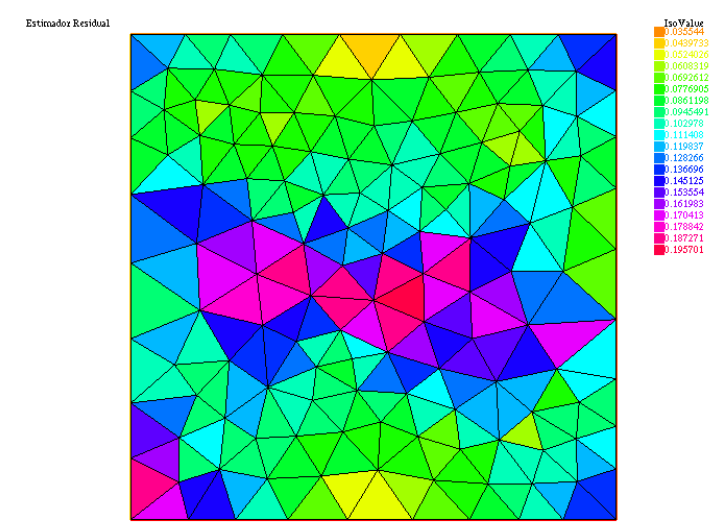

(a) Primeiro refinamento $=\{0.035544: 0.195701\}$

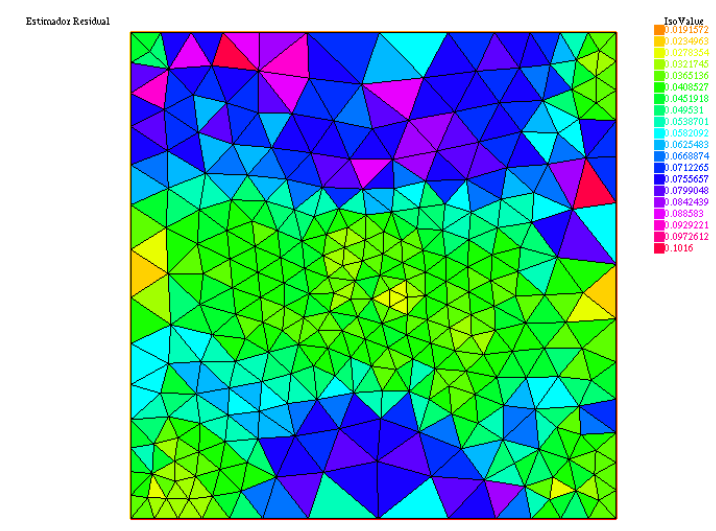

(c) Terceiro refinamento $=\{0.0191572: 0.1016\}$

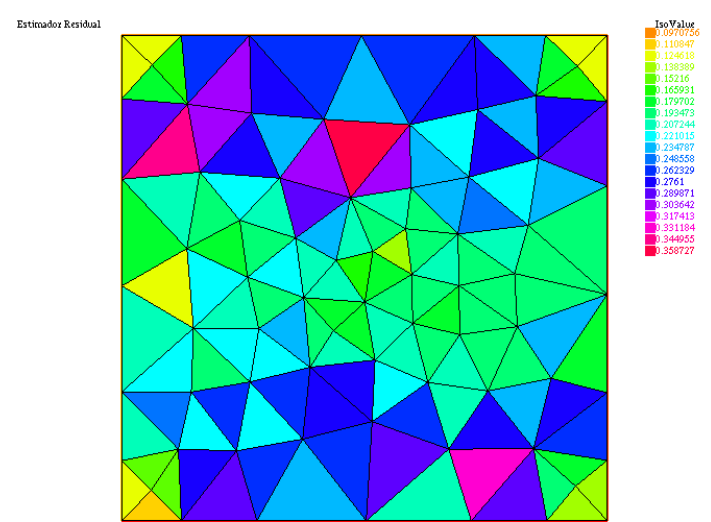

(b) Segundo refinamento $=\{0.0979756: 0.358727\}$

Figura 3.7: Comportamento do estimador de erro nas iterações de refinamento adaptativo.

As regiões onde o estimador apresenta valores maiores para o erro mudam de lugar a cada iteração pelo fato do refinamento adaptativo aumentar o número de elementos somente nas regiões onde o indicador fornece valores maiores. 
Pelos dados das Tabelas (3.1) e (3.2), observamos que o refinamento uniforme gera uma quantidade consideravelmente maior de nós em cada iteração, fazendo com que o sistema linear associado ao problema possua a dimensão do número de nós da malha. Para reduzir a quantidade de nós utilizamos o refinamento adaptativo para construir uma aproximação cujo erro L2 é mesma ordem de grandeza daquele obtido na quarta iteração do refinamento uniforme, conforme mostra a Tabela (3.3).

\begin{tabular}{|l|c|c|}
\hline & Uniforme (4 iterações) & Adaptativo (6 iterações) \\
\hline Quantidade de Nós & 7585 & 2351 \\
\hline Quantidade de Triângulos & 14848 & 4352 \\
\hline Erro na norma L2 & 0.000182 & 0.000393 \\
\hline Erro Residual & 0.259999 & 0.394425 \\
\hline$\varepsilon(\%)$ & 0.114203 & 0.173283 \\
\hline Tempo computacional $(m s)$ & 210 & 60 \\
\hline
\end{tabular}

Tabela 3.3: Comparação entre o número de nós de cada um dos refinamentos para obter um erro L2 de mesma ordem.

Observamos que a malha gerada pelo refinamento adaptativo foi capaz de obter uma aproximação com a mesma ordem de grandeza utilizando aproximadamente $30 \%$ da quantidade de nós utilizada pelo refinamento uniforme. Como a quantidade de nós implica diretamente no tempo computacional necessário para a obtenção das aproximações (pois o sistema a ser resolvido depende da quantidade de nós), observamos que o refinamento adaptativo de maneira reduz de forma significativa o esforço computacional utilizado na construção das aproximações.

\subsubsection{Problema com singularidade}

Consideramos agora o PVC

$$
\begin{cases}-\Delta u=1, & (x, y) \in \Omega \\ u(x, y)=0, & (x, y) \in \Gamma\end{cases}
$$

onde $\Omega$ é um conjunto da forma $L$-shaped, conforme descrito na Figura [3.8]:

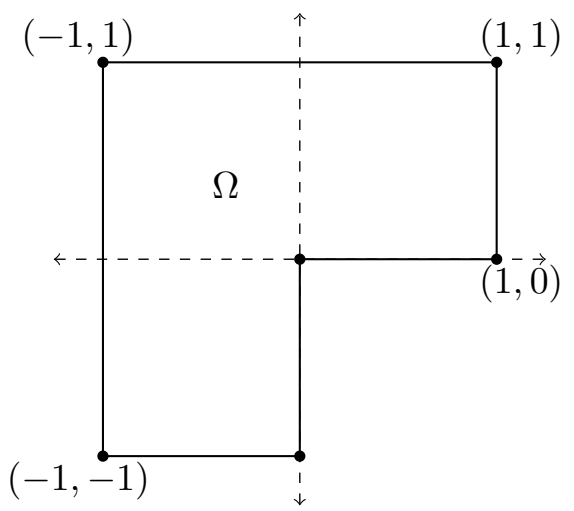

Figura 3.8: Dominio do problema

É possível mostrar que a solução deste problema ao redor da origem pode ser aproximada pela função

$$
\tilde{u}(r, \theta)=r^{\frac{2}{3}} \sin \left(\frac{(2 \theta+\pi)}{3}\right)
$$

onde $r$ representa a distância radial até a origem e $\theta$ o ângulo em relação ao eixo vertical (isto é, coordenadas polares). Como as derivadas radiais de $\tilde{u}$ não são limitadas na origem temos uma singularidade neste ponto de modo que é esperado que o erro cometido ao redor da origem seja 
maior do que o erro cometido no restante do domínio. A referência [23] faz uma discussão bastante aprofundada sobre problemas desta natureza.

Com o uso de um refinamento simples do dominio $\Omega$, obtivemos as aproximações e os estimadores residuais contidos nas Figuras (3.9(a)) e (3.9(b)).
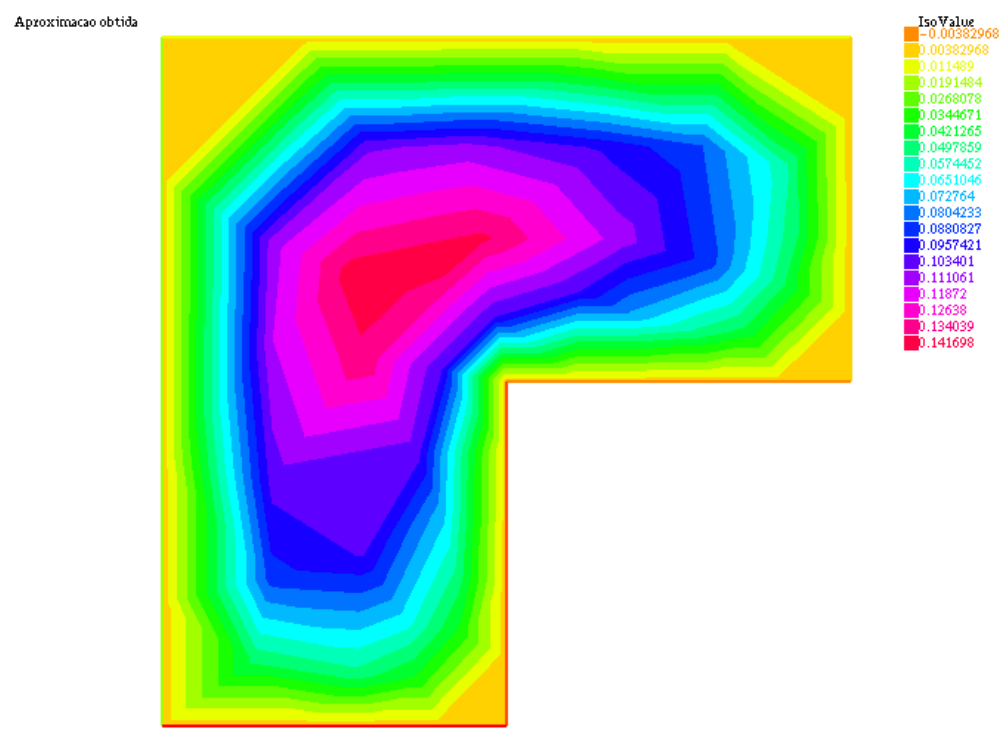

(a) Aproximação inicial $=\{0.00382968: 0.141698\}$
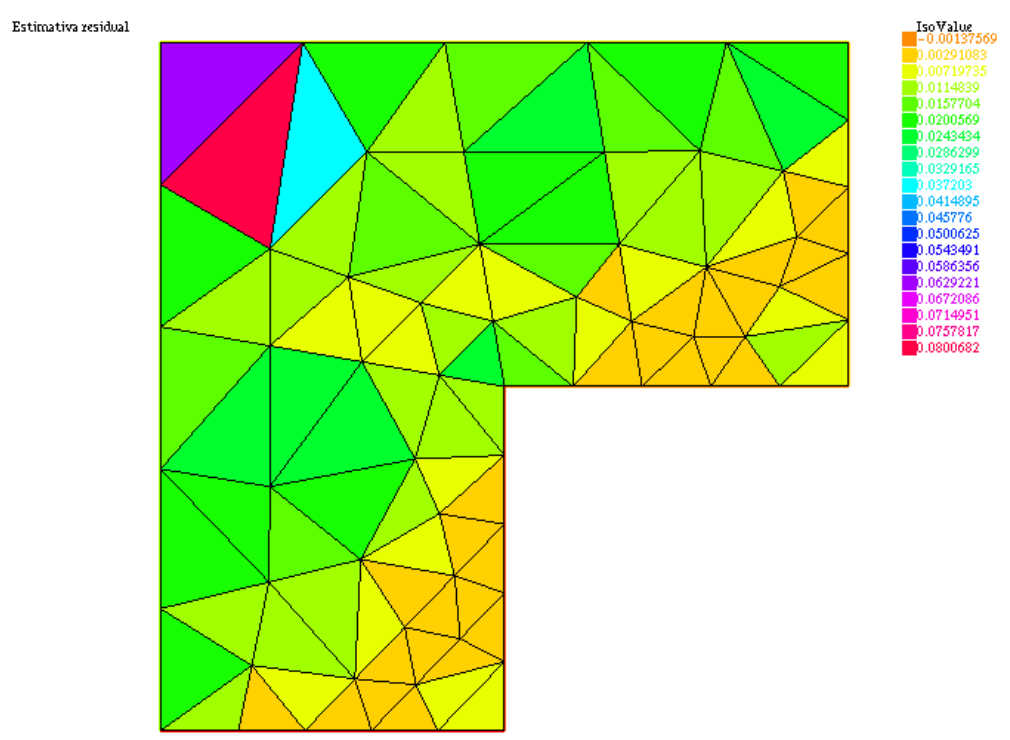

(b) Estimativa residual $=\{0.00137569: 0.0800682\}$

Figura 3.9: Resultados obtidos sobre os elementos triangulares no problema 3.17 .

Nesta aproximação inicial não foi possível identificar a necessidade de um maior número de elementos ao redor da origem. Porém, como a aproximação inicial é bastante grosseira e a solução exata do problema é considerada suave, vamos realizar algumas iterações de refinamento e analisar o comportamento das aproximações obtidas. As Figuras (3.10) e (3.11) apresentam os resultados obtidos na primeira iteração de refinamento com ambos os algoritmos de refinamento. Os dados comparativos entre as malhas obtidas encontram-se na Tabela (3.4). 


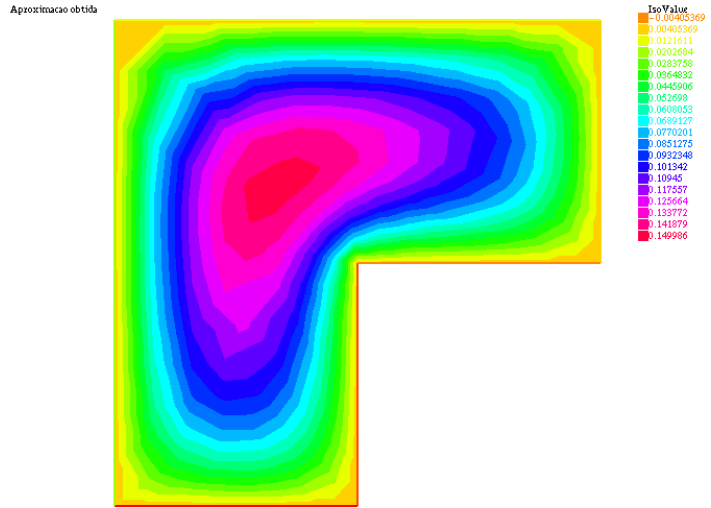

(a) Aproximação obtida $=\{0.00405369: 0.149986\}$

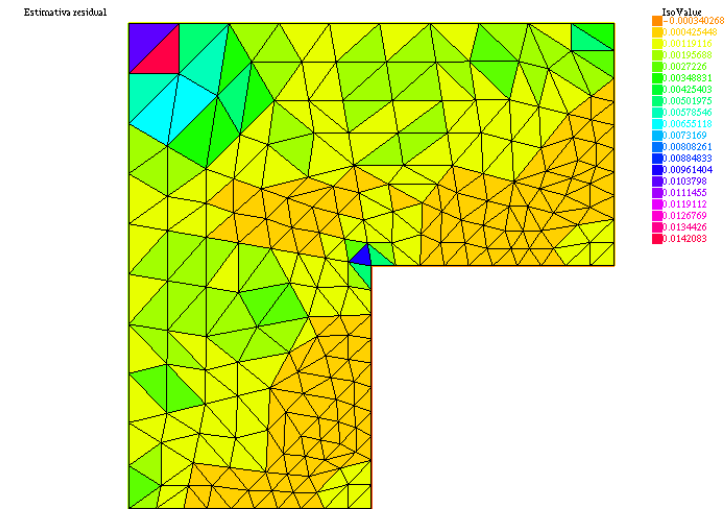

(b) Estimativa residual $=\{000340268: 0.0142083\}$

Figura 3.10: Resultados da primeira iteração do refinamento uniforme.

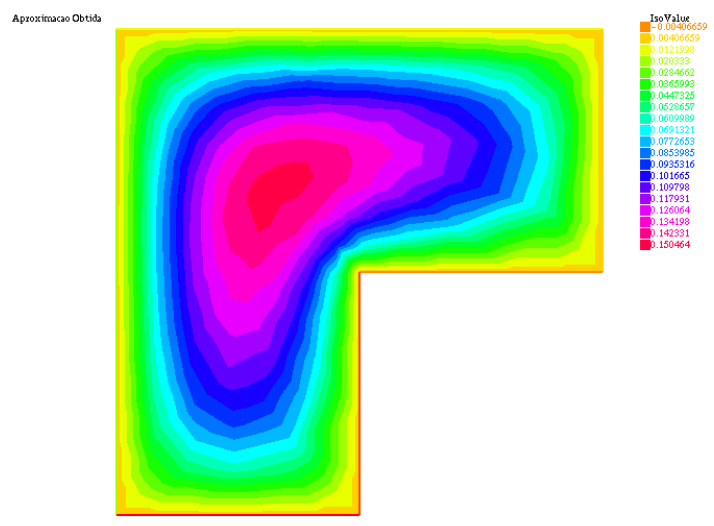

(a) Aproximação obtida $=\{0.00406659: 0.150464\}$

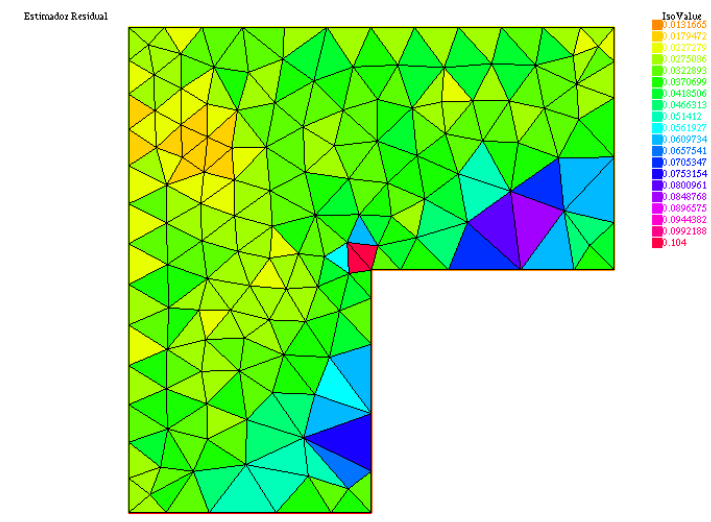

(b) Estimativa residual $=\{0.0131655: 0.104\}$

Figura 3.11: Resultados da primeira iteração do refinamento adaptativo.

\begin{tabular}{|l|c|c|}
\hline & Uniforme & Adaptativo \\
\hline Quantidade de Nós & 215 & 148 \\
\hline Quantidade de Triângulos & 368 & 249 \\
\hline Erro Residual & 0.544687 & 0.541737 \\
\hline$\varepsilon(\%)$ & 1.14936 & 1.1418 \\
\hline Tempo computacional $(m s)$ & $<<1$ & $<<1$ \\
\hline
\end{tabular}

Tabela 3.4: Comparação entre o refinamento uniforme e o refinamento adaptativo na primeira iteração de refinamento.

Como a solução exata deste problema é desconhecida vamos utilizar a estimativa residual para analisar o comportamento do erro. Na primeira iteração de refinamento observamos novamente que o algoritmo adaptativo fornece uma aproximação com o erro residual bastante próximo da aproximação obtida sobre a malha do refinamento uniforme utilizando uma quantidade de nós consideravelmente menor, reduzindo assim o esforço computacional utilizado na construção das aproximações. 
Para avaliar a convergência do método realizamos mais duas iterações de refinamento e analisamos a convergência das aproximações construidas em ambos os refinamentos (uniforme e adaptativo) por meio do erro residual cometido em cada uma das discretizações utilizadas. Pelos resultados contidos na Tabela (3.5) observamos que o erro residual é reduzido em ambos os refinamentos, indicando assim a convergência das aproximações obtidas.

\begin{tabular}{|c|c|c|}
\hline Iteração & Uniforme & Adaptativo \\
\hline$(1)$ & 0.544687 & 0.541737 \\
\hline$(2)$ & 0.298928 & 0.361026 \\
\hline$(3)$ & 0.161553 & 0.231439 \\
\hline$(4)$ & 0.0878034 & 0.146224 \\
\hline
\end{tabular}

Tabela 3.5: Erro obtido avaliado pelo estimador residual

Apesar de ambas as estratégias serem convergentes, observamos que o refinamento adaptativo reduz de forma significante a quantidade de nós utilizada na discretização, como podemos observar na Figura (3.12).

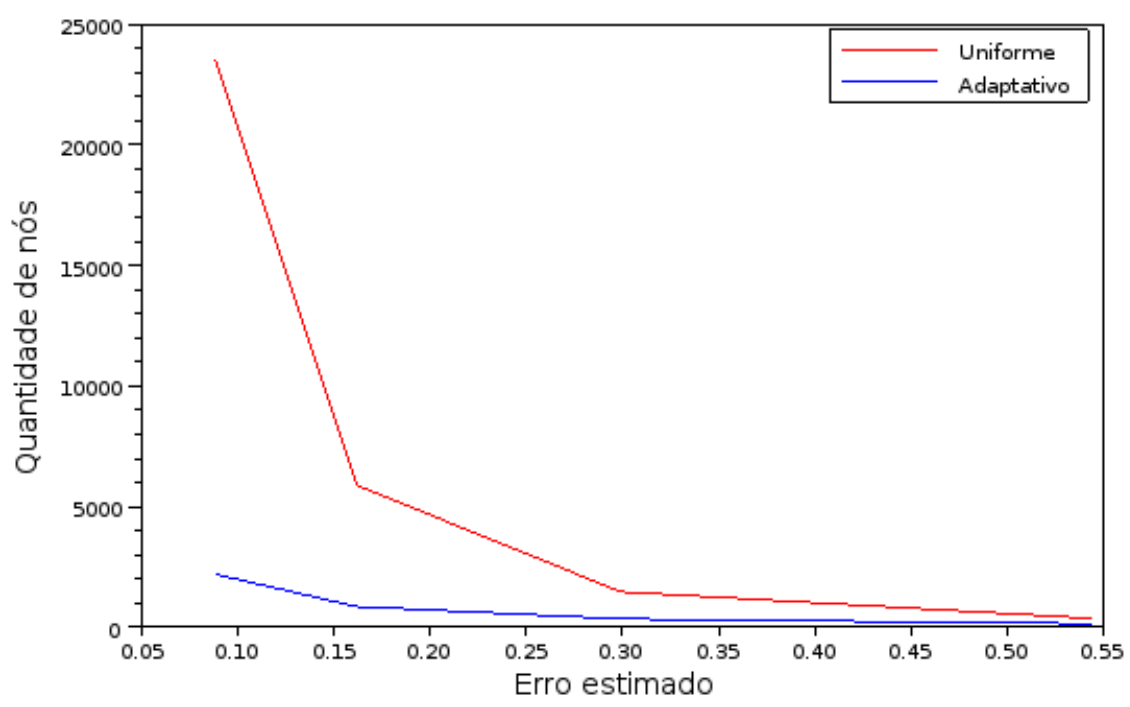

Figura 3.12: Relação entre o erro cometido e o número de nós da malha.

Tomando como exemplo a quarta iteração de refinamento, as aproximações contidas na Figura (3.13) mostram que ambos os refinamentos fornecem soluções bem próximas. No entanto, pela análise da Figura (3.12), é possível observar que o refinamento adaptativo apresenta uma redução de aproximadamente $90 \%$ no número de nós em relação a malha obtida com refinamento uniforme. 


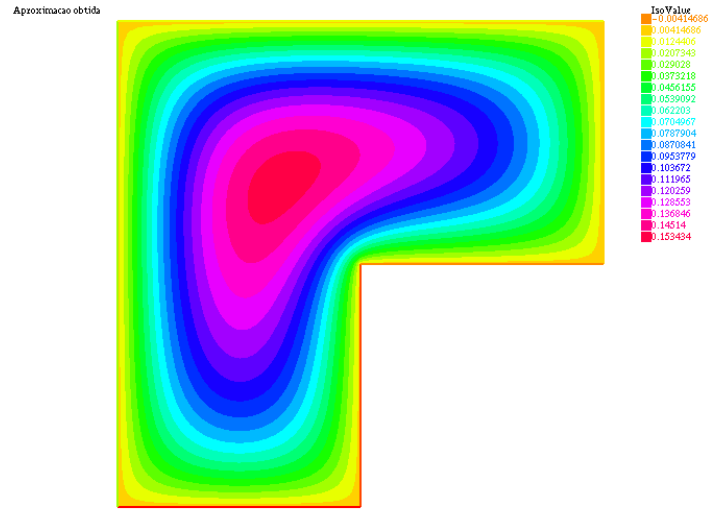

(a) Refinamento uniforme $=\{0.00414686: 0.153434\}$

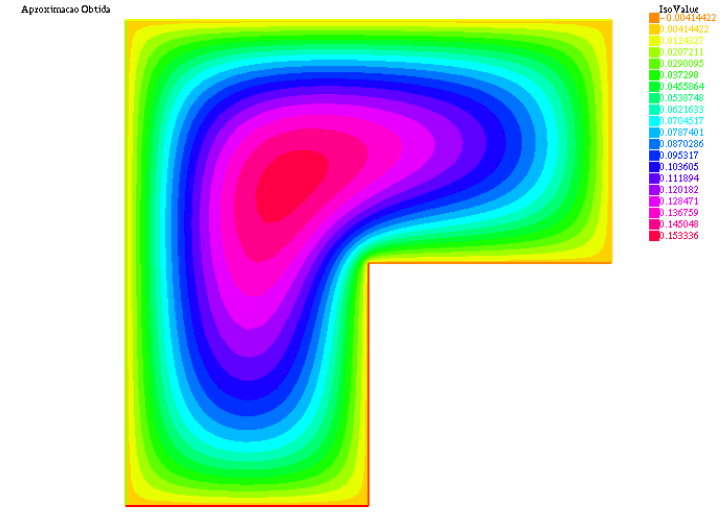

(b) Refinamento adaptativo $=\{0.0041442: 0.153336\}$

Figura 3.13: Comparativo das aproximações obtidas na quarta iteração de refinamento.

Isto mostra a utilização do refinamento adaptativo de malhas permite reduzir de maneira considerável o tempo computacional utilizado para obter as soluções numéricas do problema sem afetar a convergência do método numérico, gerando assim aproximações bastante precisas utilizando um número consideravelmente baixo de incógnitas no sistema. 


\section{Capítulo 4}

\section{Elasticidade Linear}

Neste capítulo apresentaremos a aplicação das estimativas residuais para estimar o erro cometido na aplicação do MEF em problemas envolvendo elasticidade linear. Visando simplificar a apresentação do modelo e suas respectivas hipóteses, seguiremos o conteúdo apresentado em [14] complementando com a teoria contida em [16] e [15]. O estudo será feito para pequenas deformações, de maneira que as equações envolvidas possam ser consideradas lineares.

A teoria da elasticidade relaciona as deformações sofridas por um determinado corpo com as tensões e trações aplicadas sobre o mesmo. Com isto, para obtermos as equações que regem o modelo é preciso que sejam obtidas as equações associadas a cinemática do corpo em estudo, suas relações constitutivas e as equações de equilibrio. A partir destes três componentes será obtida a formulação forte do problema de valor de contorno que fornece o deslocamento em cada ponto do domínio.

Para a aplicação do MEF iremos construir a formulação variacional associada a este método utilizando alguns resultados análogos aqueles utilizados nos problemas abordados até aqui. A grande dificuldade neste ponto reside na obtenção destes resultados análogos para o sistema de equações em estudo. A partir daí, a construção da estimativa é feita exatamente como nos casos anteriores, com a aplicação do teorema da divergência de Gauss sobre cada um dos elementos e a avaliação do residuo cometido sobre cada componente do domínio.

\subsection{As equações da elasticidade linear}

Considere $\Omega$ uma superfície poligonal e $\Gamma=\partial \Omega$ sua fronteira, de forma que $\Gamma$ é dividida em dois trechos: $\Gamma_{D}$ a região onde o deslocamento é conhecido e $\Gamma_{N}$ a região onde as tensões são conhecidas.

Considere $\vec{u}: \Omega \rightarrow \mathbb{R}^{2}$ o deslocamento ocorrido em cada ponto e $\varepsilon(\vec{u})$ o tensor de deformação do corpo dado por

$$
\varepsilon(\vec{u})=\frac{1}{2}\left(\nabla u+\nabla u^{t}\right)=\frac{1}{2}\left(\frac{\partial u_{j}}{\partial x_{i}}+\frac{\partial u_{i}}{\partial x_{j}}\right)_{1 \leq i, j \leq 2}
$$

Da relação constitutiva, consideramos que o tensor de tensões é dado por

$$
\sigma(\varepsilon)=\lambda \operatorname{tr}(\varepsilon) I+2 \mu \varepsilon
$$

onde $I \in \mathbb{R}^{2 \times 2}$ é o tensor unitário, $\operatorname{tr}(\varepsilon)$ é o traço de $\varepsilon$ e $\lambda, \mu$ são os chamados parâmetros de Lamé e dependem do coeficiente de elasticidade e de Poisson do material. Para garantir a existência e a unicidade de soluções do problema vamos assumir ${ }^{1}$ que o trecho do contorno onde o deslocamento é conhecido possui medida positiva em $\mathbb{R}$ (ou seja, o deslocamento não é conhecido somente em um conjunto enumerável de pontos, mas sim em um segmento todo da fronteira).

Denotando por $\vec{f}$ o vetor de forças de volume aplicadas sobre a placa e $\vec{g}$ o vetor de forças de tração aplicadas sobre a placa, os deslocamentos ocorridos em cada ponto da placa são obtidos pela

\footnotetext{
${ }^{1}$ Esta hipótese será utilizada pelo o Teorema (4.3.2) para mostrar que o problema admite solução única
} 
resolução do seguinte problema de valor de contorno:

$$
\left\{\begin{array}{c}
-\operatorname{div}(\sigma(\varepsilon))=\vec{f} \quad \text { em } \Omega \\
\sigma(\varepsilon(\vec{n}))=\vec{g} \quad \text { em } \Gamma_{N} \\
\vec{u}=0 \quad \operatorname{em~} \Gamma_{D}
\end{array}\right.
$$

\subsection{Formulação variacional (fraca) do problema: Minimizando a energia associada}

A formulação fraca deste problema é construida exatamente como nos problemas anteriores: multiplicamos a formulação forte por uma função de ponderação $\vec{v}$ e integramos sobre o domínio da superfície em estudo. Aplicando o teorema da Divergência de Gauss o problema descrito em sua formulação fraca é equivalente a obtermos o valor mínimo do funcional de energia associado ao modelo. Portanto, resolver o problema de valor de contorno (4.1) é equivalente à minimizar o funcional que representa a energia contida no sistema

$$
\Pi(u)=\int_{\Omega}\left[\frac{1}{2} \sigma(\vec{u}): \varepsilon(\vec{u})-\vec{f} \cdot \vec{u}\right]+\int_{\Gamma_{N}} \vec{g} \cdot \vec{u}
$$

para $\vec{u} \in H_{D}^{1}(\Omega) \times H_{D}^{1}(\Omega)$, onde $H_{D}^{1}(\Omega)$ é um espaço de Sobolev em $\Omega$ que se anula no trecho $\Gamma_{D}$ da fronteira e $\varepsilon: \sigma$ é o produto tensorial padrão, isto é

$$
\varepsilon: \sigma=\sum_{1 \leq i, j \leq 2} \varepsilon_{i, j} \sigma_{i, j}
$$

Observe que as variáveis $\varepsilon, \sigma$ e $\vec{u}$ não são independentes, mas são relacionadas pela relação constitutiva do material e pela cinemática do problema. Além disso, diferente do que ocorria para os problemas anteriores onde apenas uma função incognita era construída, estamos buscando duas funções para aproximar os deslocamentos nos dois sentidos da superfície em estudo.

Observe que o funcional energia pode ser escrito como a formulação equivalente descrita na Seção (2.2): Sendo $V=H_{D}^{1}(\Omega) \times H_{D}^{1}(\Omega)$ um espaço normado, $a(\cdot, \cdot)$ uma forma bilinear dada por

$$
a(u, u)=\int_{\Omega} \sigma(\vec{u}): \varepsilon(\vec{u})
$$

e o funcional linear $\langle f, u\rangle$ é descrito como

$$
<f, u>=\int_{\Omega} \vec{f} \cdot \vec{u}+\int_{\Gamma_{N}} \vec{g} \cdot \vec{u}
$$

Se a forma bilinear for positiva definida, limitada e coerciva então podemos aplicar o Teorema (2.2.1) e concluir que a energia mínima $\vec{u} \in H_{D}^{1}(\Omega) \times H_{D}^{1}(\Omega)$ satisfaz a igualdade

$$
\int_{\Omega} \sigma(\vec{u}): \varepsilon(\vec{u})=\int_{\Omega} \vec{f} \cdot \vec{u}+\int_{\Gamma_{N}} \vec{g} \cdot \vec{u}
$$

A solução aproximada pelo MEF será construída por meio da discretização do domínio em um conjunto de partições regulares $\mathcal{T}$, onde a aproximação $\overrightarrow{u_{\tau}} \in S_{D}^{1,0}(\mathcal{T}) \times S_{D}^{1,0}(\mathcal{T})$ é obtida de modo que satisfaça a igualdade

$$
\int_{\Omega} \sigma\left(\overrightarrow{u_{\tau}}\right): \varepsilon\left(\overrightarrow{v_{\tau}}\right)=\int_{\Omega} \vec{f} \cdot \overrightarrow{v_{\tau}}+\int_{\Gamma_{N}} \vec{g} \cdot \overrightarrow{v_{\tau}}
$$

para toda $\overrightarrow{v_{\tau}} \in S_{D}^{1,0}(\mathcal{T}) \times S_{D}^{1,0}(\mathcal{T})$ 


\subsection{Resultados auxiliares}

Apesar da estrutura do problema ser similar aquela utilizada para o problema de Poisson, não sabemos se o problema apresentado possui solução única. Para isto, iremos apresentar agora alguns importantes resultados capazes de mostrar que a forma bilinear associada ao problema é contínua e coerciva e o funcional linear é contínuo. Com isto, pelo Teorema de Lax-Milgram (2.1.15), podemos concluir que o problema possui solução única.

Teorema 4.3.1 (Primeira desigualdade de Korn). Seja $\Omega \subset \mathbb{R}^{3}$ um conjunto aberto com fronteira seccionalmente diferenciável. Assim, existe um número $c=c(\Omega)>0$ tal que

$$
\left(\int_{\Omega} \varepsilon(v): \varepsilon(v)\right)+\|v\|_{0}^{2} \geq c\|v\|_{1}^{2}
$$

para toda função $v \in H^{1}(\Omega) \times H^{1}(\Omega)$.

Sendo $H_{\Gamma}^{1}(\Omega)$ o fecho do conjunto $v \in \mathcal{C}^{\infty}(\Omega)^{2}: v(x)=0$ para $x \in \Gamma_{D}$ na norma $\|\cdot\|_{1}$, o resultado a seguir mostra que a forma bilinear associada é coerciva.

Teorema 4.3.2 (Segunda desigualdade de Korn). Seja $\Omega \subset \mathbb{R}^{2}$ um conjunto aberto com fronteira seccionalmente diferenciável e suponha que $\Gamma_{D}$ seja um conjunto de medida positiva em $\mathbb{R}$. Nestas condiçôes, existem um número positivo $c^{\prime}=c^{\prime}\left(\Omega, \Gamma_{D}\right)$ tal que

$$
\int_{\Omega} \varepsilon(v): \varepsilon(v) \geq c^{\prime}\|v\|_{1}^{2}
$$

para toda função $v \in H_{\Gamma}^{1}(\Omega)$.

As demonstrações destes resultados vão além dos objetivos deste trabalho. As referências [15] e [16] apresentam ambas as demonstrações em detalhes.

\subsection{Estimativa residual}

Assim como fizemos na estimativa residual para o problema de Poisson iremos avaliar a diferença entre a solução exata (inicialmente desconhecida) e a aproximação obtida sobre a formulação diferencial, utilizando que para cada um dos elementos $K \in \mathcal{T}$ temos que:

$$
\int_{K} \sigma\left(\vec{u}-\overrightarrow{u_{\tau}}\right): \varepsilon(\vec{v})=\int_{K} \vec{f} \cdot \vec{v}+\int_{E} \vec{g} \cdot \vec{v}-\int_{K} \sigma\left(\overrightarrow{u_{\tau}}\right): \varepsilon(\vec{v})
$$

Definimos implicitamente o resíduo cometido na aproximação como o lado direito da equação anterior, isto é

$$
\mathcal{R}\left(\overrightarrow{u_{\tau}}, \overrightarrow{v_{\tau}}\right)=\int_{K} \vec{f} \cdot \vec{v}+\int_{E} \vec{g} \cdot \vec{v}-\int_{K} \sigma\left(\overrightarrow{u_{\tau}}\right): \varepsilon(\vec{v})
$$

Sendo as aproximações são de classe $\mathcal{C}^{2}$ no interior dos elementos podemos desfazer as integrais por partes e reorganizar os termos, de modo que

$$
\mathcal{R}\left(\overrightarrow{u_{\tau}}, \vec{v}\right)=\sum_{K \in \mathcal{T}} \int_{K}\left(\vec{f}+\sigma\left(u_{\tau}\right)\right) \vec{v}+\sum_{E \in \mathcal{E}_{\tau, \Gamma_{N}}} \int_{E}\left(g-\vec{n}_{E} \sigma\right) \vec{v}-\sum_{E \in \mathcal{E}_{\tau, \Omega}} \int_{E} \mathbb{J}\left(\vec{n}_{E} \cdot \sigma\right) \vec{v}
$$

onde, sendo $K$ um elemento da partição $\mathcal{T}, E_{K}$ é o conjunto dos segmentos que compõem o elemento $K, \vec{n}_{E}$ o vetor normal associado ao segmento $\mathrm{E}, \mathcal{E}_{\tau, \Omega}$ e $\mathcal{E}_{\tau, \Gamma_{N}}$ os segmentos que compõem os elementos e estão contidos no interior do domínio e na fronteira $\Gamma_{N}$, respectivamente e $\mathbb{J}$ representa o salto cometido pela solução através das bordas de dois elementos adjacentes.

Observe que a forma bilinear $\int_{\Omega} \sigma(\vec{u}): \varepsilon(\vec{u})$ possui as mesmas características da forma $\int_{\Omega} \nabla u \cdot \nabla w$ utilizada na formulação do resíduo para o problema de Poisson. Desta forma, é possível mostrar 
que o Teorema (3.2.1) pode ser generalizado para o problema em estudo e que o erro cometido na aproximação $\overrightarrow{u_{\tau}}$ é limitado pela norma do resíduo no espaço dual de $H_{D}^{1}(\Omega) \times H_{D}^{1}(\Omega)$.

Assim seguimos os mesmos passos utilizados na construção do estimador residual para o problema de Poisson definindo o resíduo interior e o resíduo do bordo associado a cada elemento $K \in \mathcal{T}$ como

$$
R_{K}\left(u_{\tau}\right)=\vec{f}+\operatorname{div}\left(\sigma\left(\overrightarrow{u_{\tau}}\right)\right)
$$

$\mathrm{e}$

$$
R_{E}\left(u_{\tau}\right)= \begin{cases}-\mathbb{J}_{E}\left(\vec{n}_{E} \cdot \sigma\left(\overrightarrow{u_{\tau}}\right)\right), & \text { se } E \in \mathcal{E}_{\tau, \Omega} \\ \vec{g}-\vec{n}_{E} \cdot \sigma\left(\overrightarrow{u_{\tau}}\right), & \text { se } E \in \mathcal{E}_{\tau, \Gamma_{N}} \\ 0, & \text { se } E \in \mathcal{E}_{\tau, \Gamma_{D}}\end{cases}
$$

respectivamente. Com isto o resíduo pode ser reescrito como

$$
\mathcal{R}\left(u_{\tau}, v\right)=\sum_{K \in \mathcal{T}} \int_{K} R_{K}\left(u_{\tau}\right) v+\sum_{E \in \mathcal{E}_{\tau}} \int_{E} R_{E}\left(u_{\tau}\right) v
$$

e, de maneira análoga ao que fizemos no problema de Poisson ${ }^{2}$, podemos estimar o resíduo em cada elemento $K \in \mathcal{T}$ como:

$\eta_{R, K}=\left\{h_{K}^{2}\left\|f_{\tau}+\operatorname{div}\left(\sigma\left(u_{\tau}\right)\right)\right\|_{K}^{2}+\frac{1}{2} \sum_{E \in E_{K} \cap \mathcal{E}_{\tau, \Omega}} h_{E}\left\|J_{E}\left(\vec{n} \cdot \sigma\left(u_{\tau}\right)\right)\right\|_{E}^{2}+\sum_{E \in E_{K} \cap \mathcal{E}_{\tau, \Gamma_{N}}} h_{E}\left\|g-\vec{n} \cdot \sigma\left(u_{\tau}\right)\right\|_{E}^{2}\right\}^{\frac{1}{2}}$

onde $\|\cdot\|_{K}$ e $\|\cdot\|_{E}$ representam a norma $L^{2}$ nos elementos $K \in \mathcal{T}$ e nas arestas $E \in \mathcal{E}_{\tau}$, respectivamente.

A implementação computacional do estimador residual possui estrutura muito semelhante aquela utilizada no problema de Poisson, modificando apenas o operador a ser integrado em cada uma das componentes da estimativa.

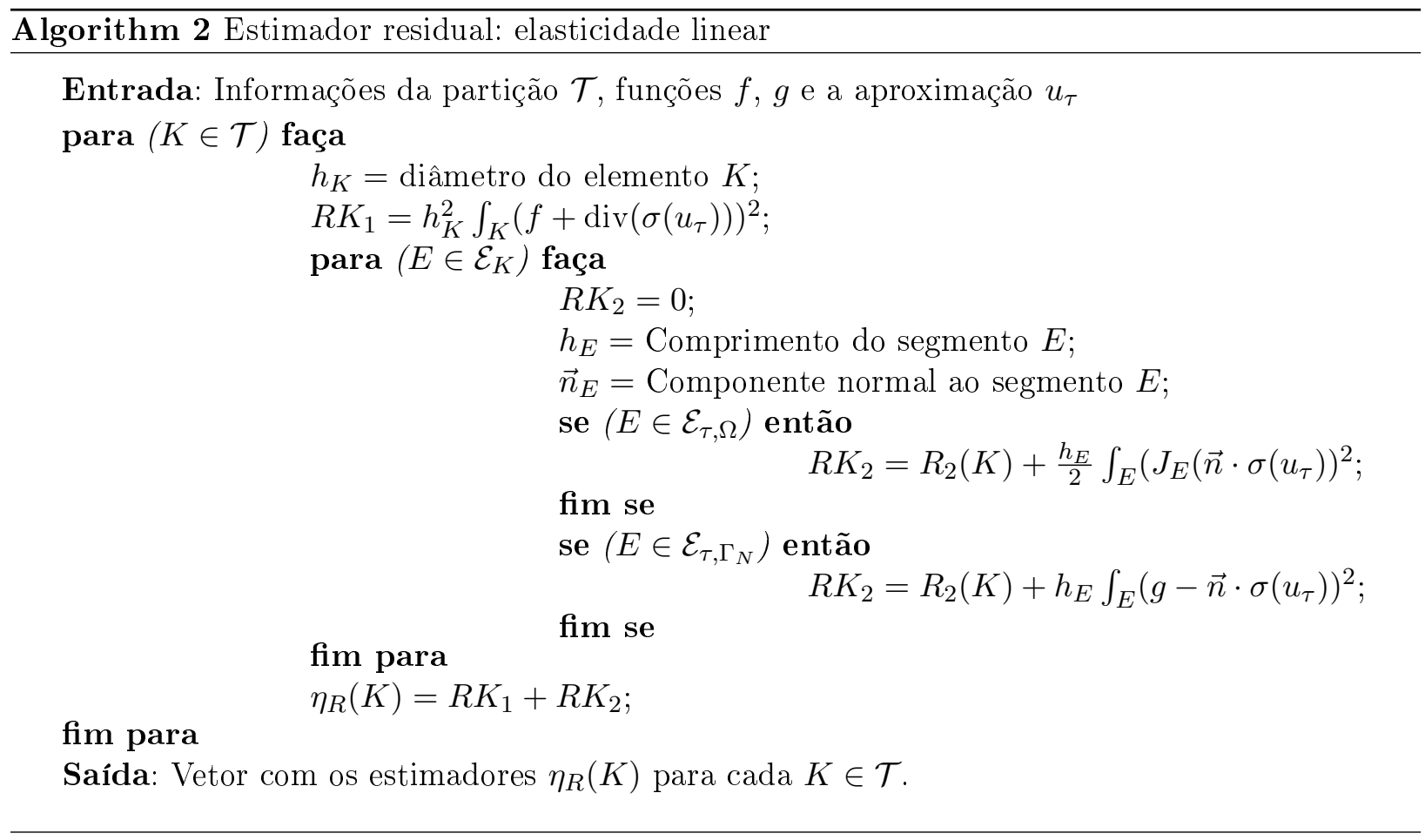

\footnotetext{
${ }^{2}$ A demonstração completa encontra-se no Apêndice A.
} 


\subsection{Simulações Numéricas}

Considere uma fina placa de aluminio com um furo circular em seu centro ${ }^{3}$ e sua extremidade inferior fixa e que sofre a ação de uma força constante de $5 \mathrm{kN}$ sobre a extremidade superior da placa. Sendo o módulo de Young $E=70 G P a$ e o coeficiente de Poisson $\nu=0.28$, vamos analisar a deformação sofrida pela placa.

Vamos utilizar inicialmente uma malha contendo 10 pontos em cada um dos trechos que compõem o contorno externo e 30 pontos no contorno que representa o furo central, conforme descrito na Figura (4.1):

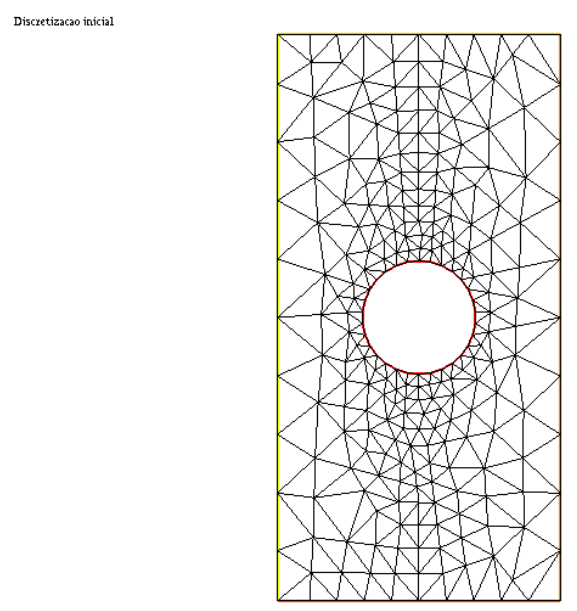

Figura 4.1: Malha inicial.

Utilizando funções interpoladoras de segunda ordem obtivemos as aproximações e o estimador residual descritos nas Figuras (4.2(a)) e (4.2(b)).
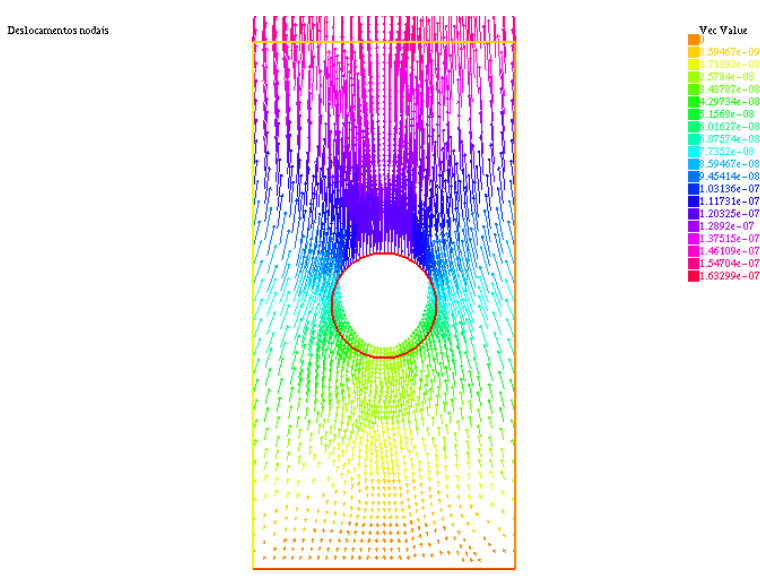

(a) Aproximações obtidas $=\{0: 1.63299 \mathrm{e}-07\}$

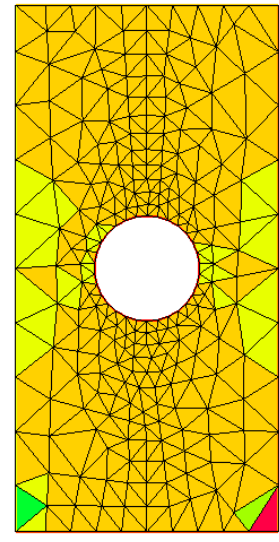

(b) Estimador residual $=\{14443.4: 534591\}$

Figura 4.2: Resultados obtidos na malha inicial.

Os resultados obtidos condizem com o comportamento físico esperado: a placa deslocou-se na direção da força constante, de modo a deformar a circunferência contida em seu interior. Para visualizar a deformação ocorrida na chapa iremos aplicar os deslocamentos nodais obtidos em cada um dos pontos da malha de estudo, obtendo os resultados da Figura (4.3).

\footnotetext{
${ }^{3} \mathrm{O}$ furo central será aproximado por um polígono, de modo que toda a teoria desenvolvida até aqui poderá ser aplicada ao problema.
} 


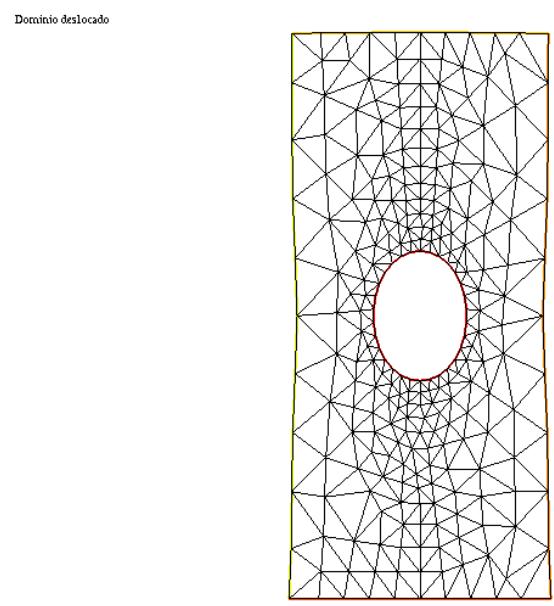

Figura 4.3: Deformação sofrida pela chapa (fator de escala $10^{6}$ ).

Para aumentar a qualidade das aproximações obtidas realizamos mais três iterações de refinamento de malha e apresentamos os resultados obtidos na terceira iteração nas Figuras (4.4) e (4.5). Como a solução exata do problema é desconhecida, utilizamos o erro estimado pelo resíduo para avaliar a convergência das aproximações, conforme mostra a Tabela (4.1).

\begin{tabular}{|c|c|c|c|c|}
\hline & \multicolumn{2}{|c|}{ Uniforme } & \multicolumn{2}{c|}{ Adaptativo } \\
\hline Iteração & Erro estimado & Tempo $(s)$ & Erro estimado & Tempo $(s)$ \\
\hline$(1)$ & 795.331 & 0.37 & 714.263 & 0.24 \\
\hline$(2)$ & 460.898 & 2.12 & 385.689 & 0.45 \\
\hline$(3)$ & 272.076 & 10.06 & 218.838 & 1.06 \\
\hline
\end{tabular}

Tabela 4.1: Erro estimado pelo estimador residual em cada uma das iterações de refinamento.

Como era esperado, observa-se que o estimador residual indica que o erro é reduzido a cada iteração do refinamento de malhas, indicando assim que as aproximações obtidas convergem para a solução do problema contínuo.

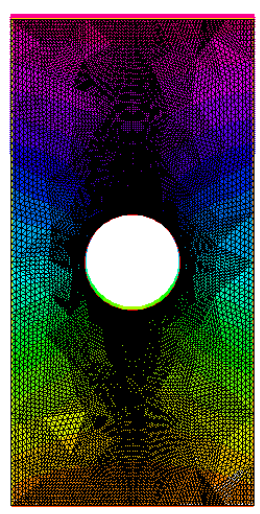

(a) Deslocamento obtido $=\{0: 1.63455 \mathrm{e}-07\}$

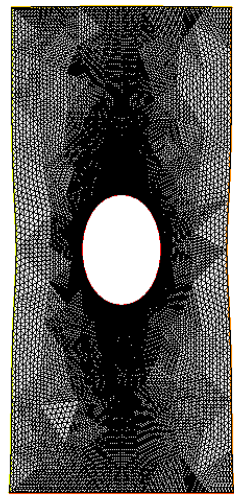

(b) Deformação na placa

Figura 4.4: Aproximação obtida na terceira iteração do refinamento uniforme. 


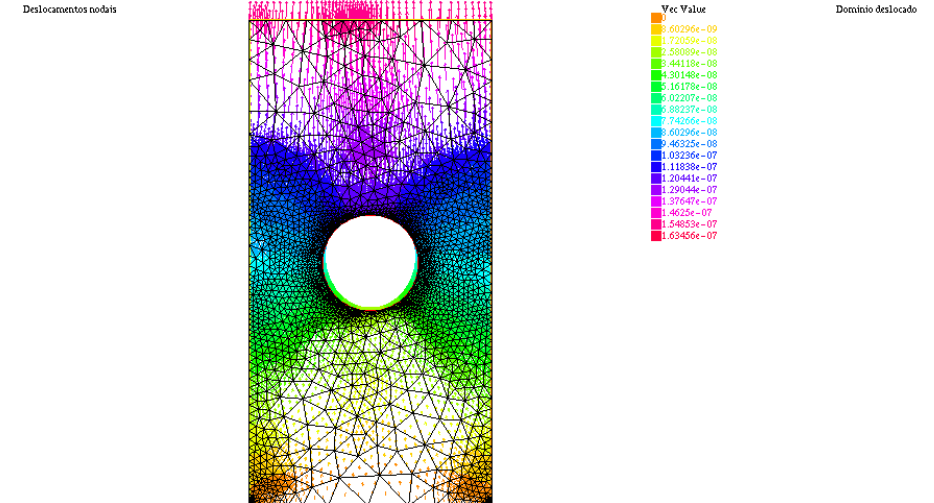

(a) Deslocamento obtido $=\{0: 1.63455 \mathrm{e}-07\}$

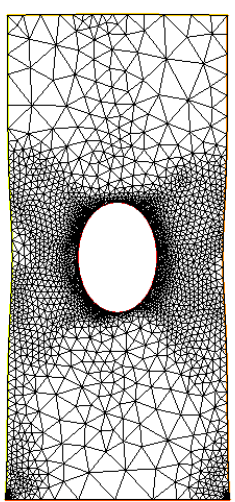

(b) Deformação na placa

Figura 4.5: Aproximação obtida na terceira iteração do refinamento adaptativo.

Observe que a quantidade de nós do terceiro refinamento uniforme é tão grande que a imagem fica bastante escura com a visualização dos elementos triangulares, enquanto que o refinamento adaptativo apresenta somente algumas regiões com um maior número de elementos. A Figura (4.6) relaciona o erro cometido nas aproximações com a quantidade de nós utilizados.

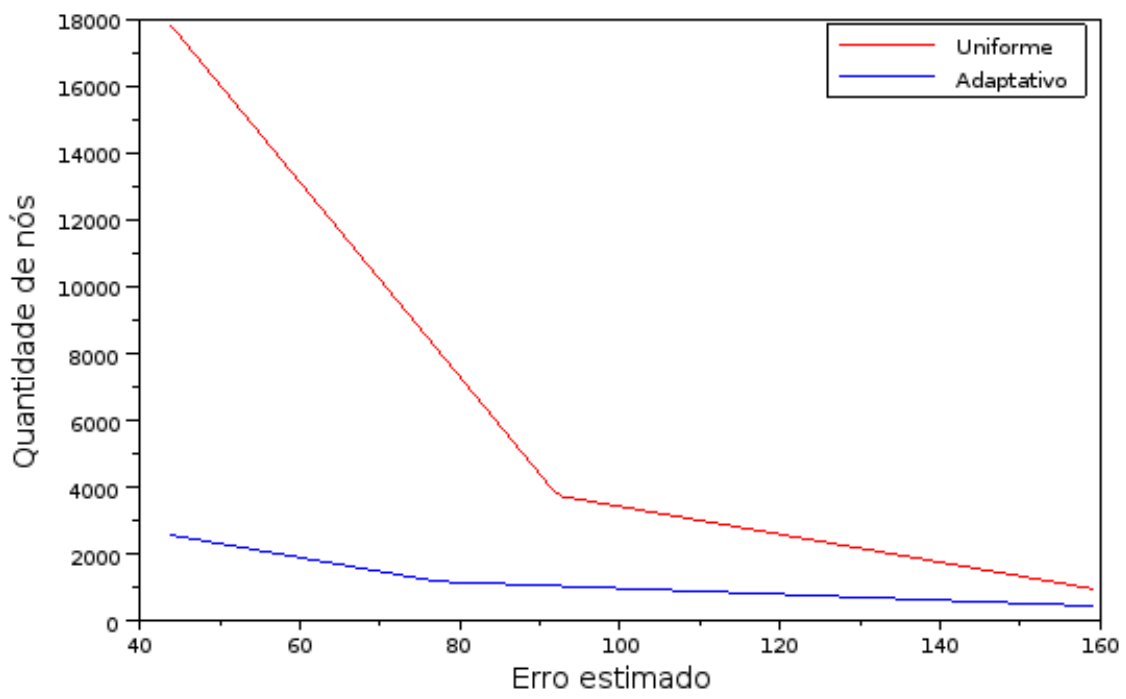

Figura 4.6: Relação entre o erro cometido e o número de nós da malha.

Assim como no problema de Poisson, observa-se que o uso do refinamento adaptativo é capaz de reduzir de maneira considerável o número de incógnitas do sistema, diminuindo assim o esforço computacional necessário para a construção das aproximações.

Por fim, analisando o comportamento do estimador do erro na terceira iteração de refinamento, cujos resíduos encontram-se na Figura (4.7), observamos as estimativas locais obtidas na malha gerada pelo refinamento uniforme encontram-se numa faixa de valores muito maior do que aqueles obtidos na malha gerada pelo refinamento adaptativo. Isto mostra que o erro cometido no interior do domínio varia de uma maneira mais uniforme na malha gerada pelo refinamento adaptativo, impedindo assim que determinadas regiões possuam o erro muito maior em relação ao restante do domínio. 

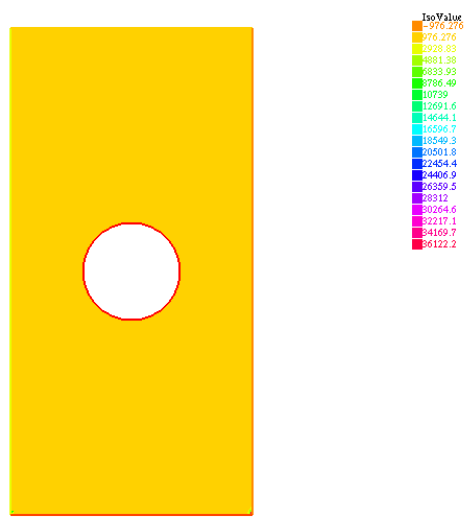

(a) Refinamento uniforme $=\{976.276: 36122.2\}$

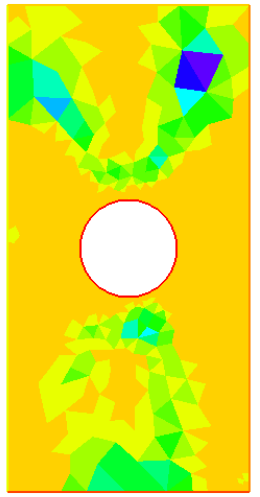

(b) Refinamento adaptativo $=\{28.0085: 1037.48\}$

Figura 4.7: Comparação dos resíduos obtidos na terceira iteração de refinamento. 


\section{Capítulo 5}

\section{Conclusões}

Nosso objetivo neste trabalho foi apresentar em detalhes a construção da estimativa de erro baseada no resíduo entre o problema contínuo e o problema discreto resolvido pelo MEF proposta nos trabalhos [1], [2], [3] e [4] e, por meio deste estimador, construir e analisar o comportamento de um algoritmo adaptativo para a construção das discretizações do domínio, de maneira a distribuir um maior número de elementos nas regiões onde o erro cometido foi maior. A escolha deste estimador deve-se à sua fácil implementação e robustez, características que fazem com que ele seja utilizado muitas vezes como parâmetro para a avaliação de outros estimadores.

Partindo da formulação variacional dos problemas em estudo, construímos o estimador residual associado a cada um dos problemas e observamos que o estimador apresentou todas as características esperadas: fácil implementação (utiliza somente os dados da discretização do domínio, as informações do contorno e as aproximações obtidas) e eficácia na identificação das regiões do domínio onde o erro cometido era maior com relação ao restante do domínio. Além disso, com o uso dos estimadores locais foi possível avaliar o erro total cometido na aproximação, o que nos permite validar também a convergência do método numérico utilizando apenas os dados do problema e as aproximações obtidas.

Com a aplicação do algoritmo adaptativo para a construção das malhas foi possível observar que a quantidade de nós da malha pode ser reduzida de maneira expressiva sem afetar a convergência do método numérico. Ou seja, o erro cometido pode ser reduzido sem a necessidade de um aumento expressivo do número de incógnitas do problema, equilibrando assim a redução no erro cometido pelo aumento no esforço computacional a ser realizado.

O estudo da aplicação da estimativa residual e do algoritmo adaptativo nos problemas envolvendo elasticidade linear mostram que a teoria pode ser aplicadade forma análoga à problemas elípticos mais gerais. Isto reforça o fato do estimador residual ser uma boa ferramenta no estudo do comportamento do erro cometido nas aproximações pelo MEF. 
CONCLUSÕES 


\section{Apêndice A}

\section{Construção do estimador residual para o problema de Elasticidade Linear}

Neste apêndice apresentaremos a construção do estimador residual para o problema de Elasticidade Linear apresentado na Seção 4.4 .

\section{A.1 Construção do estimador}

Partindo da equação que representa o resíduo utilizando o fato que as aproximações são de classe $\mathcal{C}^{2}$ no interior de cada um dos elementos (Equação 4.4):

$$
\mathcal{R}\left(\overrightarrow{u_{\tau}}, \vec{v}\right)=\sum_{K \in \mathcal{T}} \int_{K}\left(\vec{f}+\sigma\left(u_{\tau}\right)\right) \vec{v}+\sum_{E \in \mathcal{E}_{\tau, \Gamma}} \int_{E}\left(g-\vec{n}_{E} \sigma\right) \vec{v}-\sum_{E \in \mathcal{E}_{\tau, \Omega}} \int_{E} \mathbb{J}\left(\vec{n}_{E} \cdot \sigma\right) \vec{v}
$$

e das definições do resíduo interior e o resíduo do bordo associado a cada elemento $K \in \mathcal{T}$ como

$$
R_{K}\left(u_{\tau}\right)=\vec{f}+\operatorname{div}\left(\sigma\left(\overrightarrow{u_{\tau}}\right)\right)
$$

e

$$
R_{E}\left(u_{\tau}\right)=\left\{\begin{array}{lr}
-\mathbb{J}_{E}\left(\vec{n}_{E} \cdot \sigma\left(\overrightarrow{u_{\tau}}\right)\right), & \text { se } E \in \mathcal{E}_{\tau, \Omega} \\
\vec{g}-\vec{n}_{E} \cdot \sigma\left(\overrightarrow{u_{\tau}}\right), & \text { se } E \in \mathcal{E}_{\tau, \Gamma_{N}} \\
0, & \text { se } E \in \mathcal{E}_{\tau, \Gamma_{D}}
\end{array}\right.
$$

podemos substituir (A.2) e (A.3) em (A.1), de forma a obter que:

$$
\mathcal{R}\left(u_{\tau}, v\right)=\sum_{K \in \mathcal{T}} \int_{K} R_{K}\left(u_{\tau}\right) v+\sum_{E \in \mathcal{E}_{\tau}} \int_{E} R_{E}\left(u_{\tau}\right) v
$$

Note que a equação (A.4) possui as mesmas propriedades da equação (3.13), pois a forma bilinear $\int_{\Omega} \sigma(\vec{u}): \varepsilon(\vec{u})$ possui as mesmas características da forma $\int_{\Omega} \nabla u \cdot \nabla w$ utilizada na resolução do problema de Poisson. Isto permite que sigamos os mesmos passos da demonstração, isto é, utilizando a ortogonalidade de Galerkin podemos escrever que:

$$
\mathcal{R}\left(u_{\tau}, v\right)=\mathcal{R}\left(u_{\tau}, v\right)-\underbrace{\mathcal{R}\left(u_{\tau}, v_{\tau}\right)}_{=0}=\sum_{K \in \mathcal{T}} \int_{K} R_{K}\left(u_{\tau}\right)\left(v-v_{\tau}\right)+\sum_{E \in \mathcal{E}_{\tau}} \int_{E} R_{E}\left(u_{\tau}\right)\left(v-v_{\tau}\right)
$$

Considerando uma função arbitrária $v \in H_{D}^{1}(\Omega) \times H_{D}^{1}(\Omega)$, seja $v_{\tau}=I_{\tau} v$ sua projeção pelo operador de quasi-interpolação (2.18). Utilizando a desigualdade de Cauchy-Schwarz para integrais e as 
estimativas do operador podemos estimar o resíduo como

$$
\begin{aligned}
\mathcal{R}\left(u_{\tau}, v\right) & =\sum_{K \in \mathcal{T}} \int_{K} R_{K}\left(u_{\tau}\right)\left(v-I_{\tau} v\right)+\sum_{E \in \mathcal{E}_{\tau}} \int_{E} R_{E}\left(u_{\tau}\right)\left(v-I_{\tau} v\right) \\
& \leq \sum_{K \in \mathcal{T}}\left\|R_{K}\left(u_{\tau}\right)\right\|_{K} \underbrace{\left\|v-I_{\tau} v\right\|_{K}}_{\leq \alpha h_{K}\|v\|_{H_{K}^{1}}}+\sum_{E \in \mathcal{E}_{\tau}}\left\|R_{E}\left(u_{\tau}\right)\right\| \underbrace{\left\|v-I_{\tau} v\right\|_{E}}_{\beta \sqrt{h_{E}}\|v\|_{H_{E}^{1}}} \\
& \leq \sum_{K \in \mathcal{T}}\left\|R_{K}\left(u_{\tau}\right)\right\|_{K} \cdot \alpha h_{K}\|v\|_{1, \tilde{v}_{K}}+\sum_{E \in \mathcal{E}_{\tau}}\left\|R_{E}\left(u_{\tau}\right)\right\|_{E} \cdot \beta \sqrt{h_{E}}\|v\|_{1, \tilde{v}_{E}}
\end{aligned}
$$

onde, apenas para simplificar a notação, escrevemos $H_{K}^{1}=H^{1}\left(\tilde{\omega}_{K}\right) \times H^{1}\left(\tilde{\omega}_{K}\right)$ e $H_{E}^{1}=H^{1}\left(\tilde{\omega}_{E}\right) \times$ $H^{1}\left(\tilde{\omega}_{E}\right)$. Utilizando a desigualdade de Cauchy-Schwarz para soma, temos que

$$
\begin{aligned}
\mathcal{R}\left(u_{\tau}, v\right) \leq \max _{\alpha, \beta} & \left\{\sum_{K \in \mathcal{T}} h_{K}^{2}\left\|R_{K}\left(u_{\tau}\right)\right\|_{K}^{2}+\sum_{E \in \mathcal{E}_{\tau}} h_{E}\left\|R_{E}\left(u_{\tau}\right)\right\|_{E}^{2}\right\}^{\frac{1}{2}} . \\
& \cdot\left\{\sum_{K \in \mathcal{T}}\|v\|_{1, \tilde{v}_{K}}^{2}+\sum_{E \in \mathcal{E}_{\tau}}\|v\|_{1, \tilde{v}_{E}}^{2}\right\}^{\frac{1}{2}}
\end{aligned}
$$

e, por fim, utilizando o fato que a malha $\mathcal{T}$ é regular, podemos escrever que

$$
\left\{\sum_{K \in \mathcal{T}}\|v\|_{1, \tilde{v}_{K}}^{2}+\sum_{E \in \mathcal{E}_{\tau}}\|v\|_{1, \tilde{v}_{E}}^{2}\right\}^{\frac{1}{2}} \leq c\|v\|_{1}
$$

onde $c$ é uma constante que depende apenas da malha utilizada. Assim, sendo a forma bilinear coerciva, concluimos que

$$
\left\|u-u_{\tau}\right\|_{1} \leq C\left(\sum_{K \in \mathcal{T}} h_{K}^{2}\left\|R_{K}\left(u_{\tau}\right)\right\|_{K}^{2}+\sum_{E \in \mathcal{E}_{\tau}} h_{E}\left\|R_{E}\left(u_{\tau}\right)\right\|_{E}^{2}\right)^{\frac{1}{2}}
$$

onde $C$ depende somente da malha de elementos em estudo. Desta forma, concluímos o seguinte resultado:

Teorema A.1.1 (Estimador de Erro Residual a Posteriori para Elasticidade Linear). Sejam $u \in$ $H_{D}^{1}(\Omega) \times H_{D}^{1}(\Omega)$ e $u_{\tau} \in S_{D}^{1,0}(\mathcal{T}) \times S_{D}^{1,0}(\mathcal{T})$ as soluçôes dos problemas (4.2) e (4.3), respectivamente. Para cada elemento $K \in \mathcal{T}$, definimos o estimador de erro residual a posteriori $\eta_{K}$ como:

$$
\begin{gathered}
\left.\eta_{K}^{2}=h_{K}^{2} \| \vec{f}+\sigma\left(u_{\tau}\right)\right) \vec{v}\left\|_{K}^{2}+\frac{1}{2} \sum_{E \in \mathcal{E}_{K} \cap \mathcal{E}_{\tau, \Omega}} h_{E}\right\| \mathbb{J}_{E}\left(\vec{n}_{E} \cdot \sigma\left(\overrightarrow{u_{\tau}}\right)\right) \|_{E}^{2} \\
+\sum_{E \in \mathcal{E}_{K} \cap \mathcal{E}_{\tau, \Gamma}} h_{E}\left\|\vec{g}-\vec{n}_{E} \cdot \sigma\left(\overrightarrow{u_{\tau}}\right)\right\|_{E}^{2}
\end{gathered}
$$

Sob estas condições, temos que

$$
\left\|u-u_{\tau}\right\|_{1} \leq C\left(\sum_{K \in \mathcal{T}} \eta_{K}^{2}\right)^{\frac{1}{2}}
$$

onde $C$ é uma constante que depende apenas da malha utilizada. 


\section{Apêndice B}

\section{Códigos utilizados}

Apresentamos a seguir os códigos utilizados nas simulações nos capítulos 3 e 4 .

Listing B.1: testes_cap3/teste1.edp

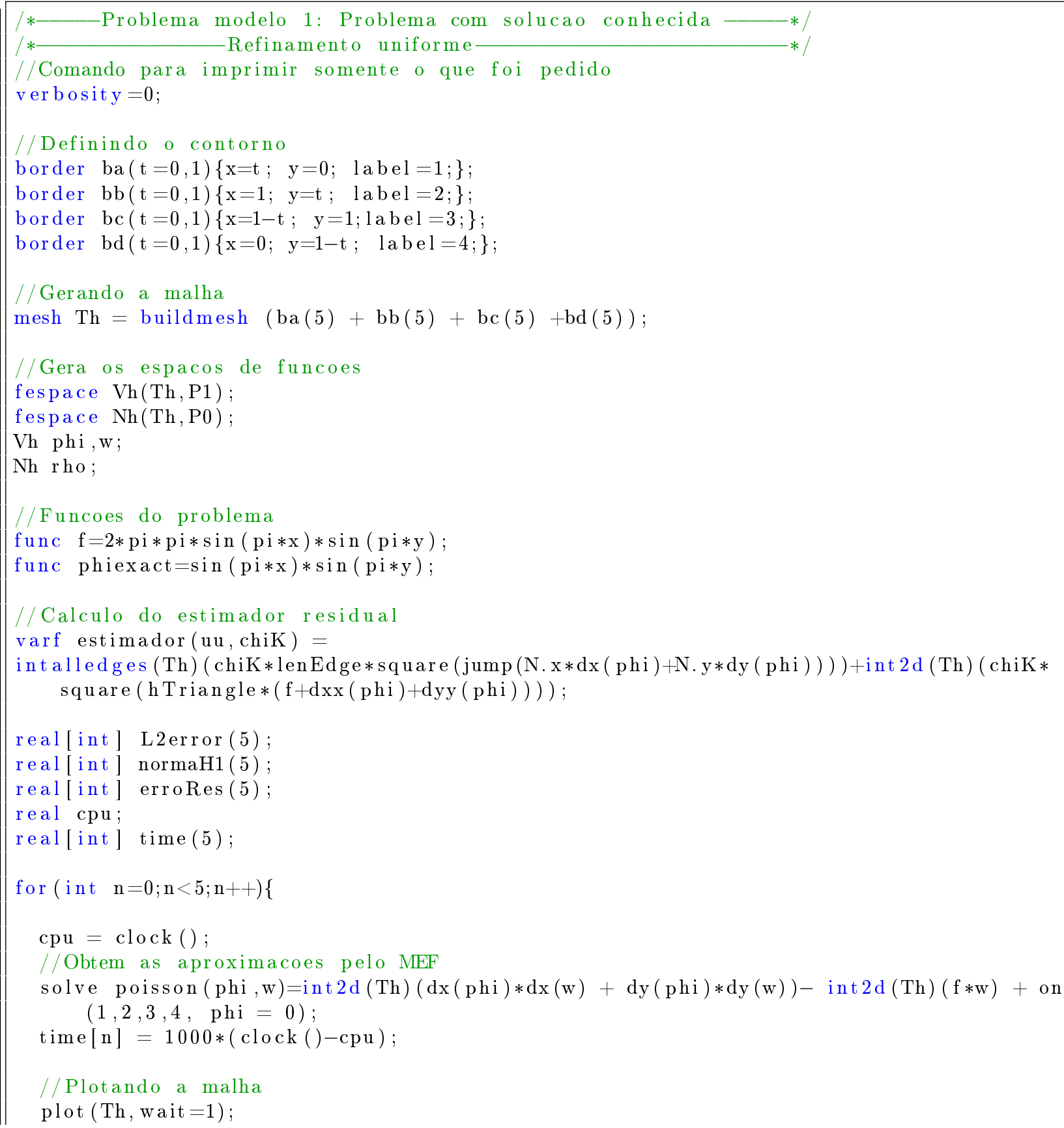




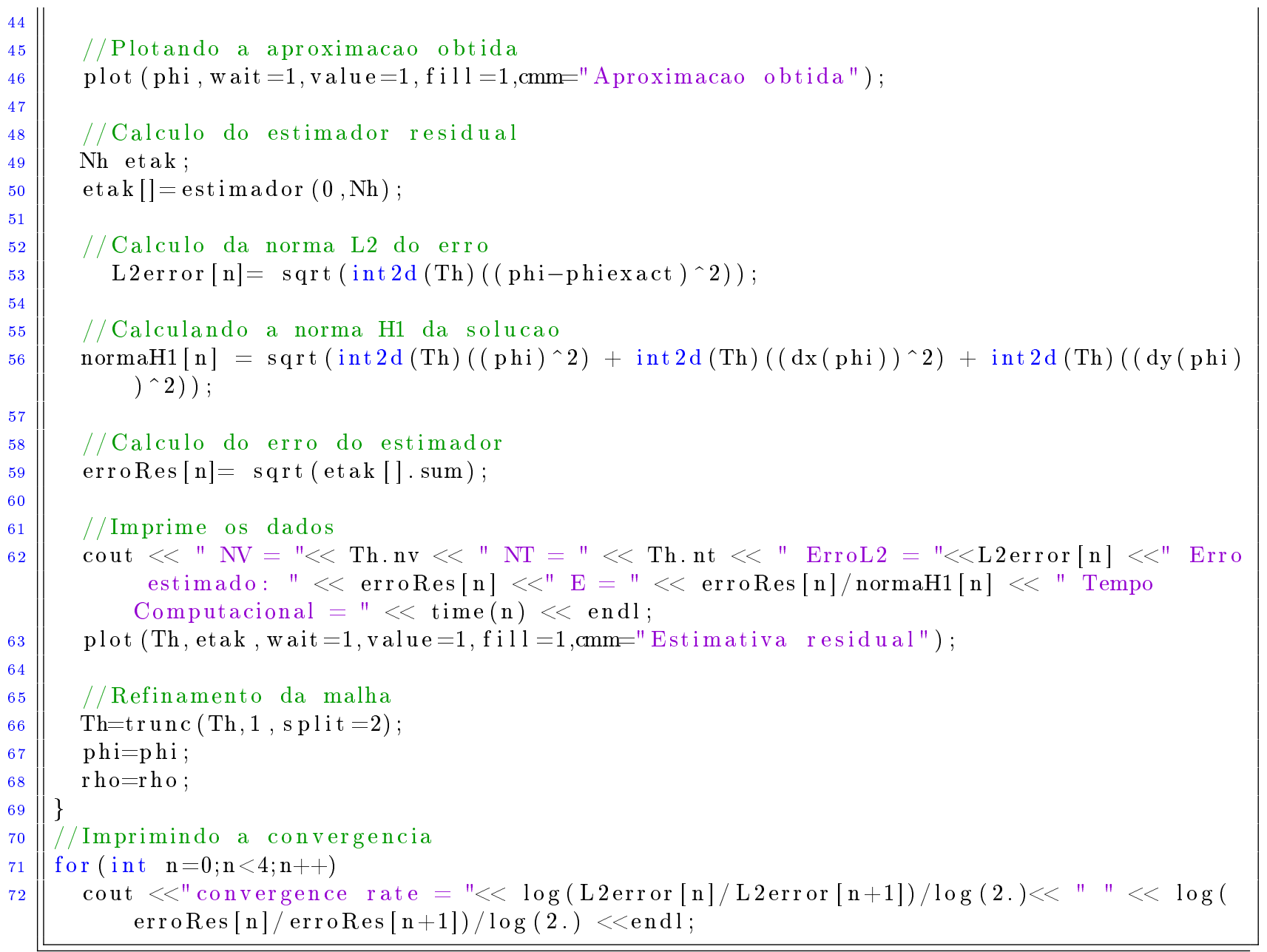

Listing B.2: testes_cap3/teste1_adapt.edp

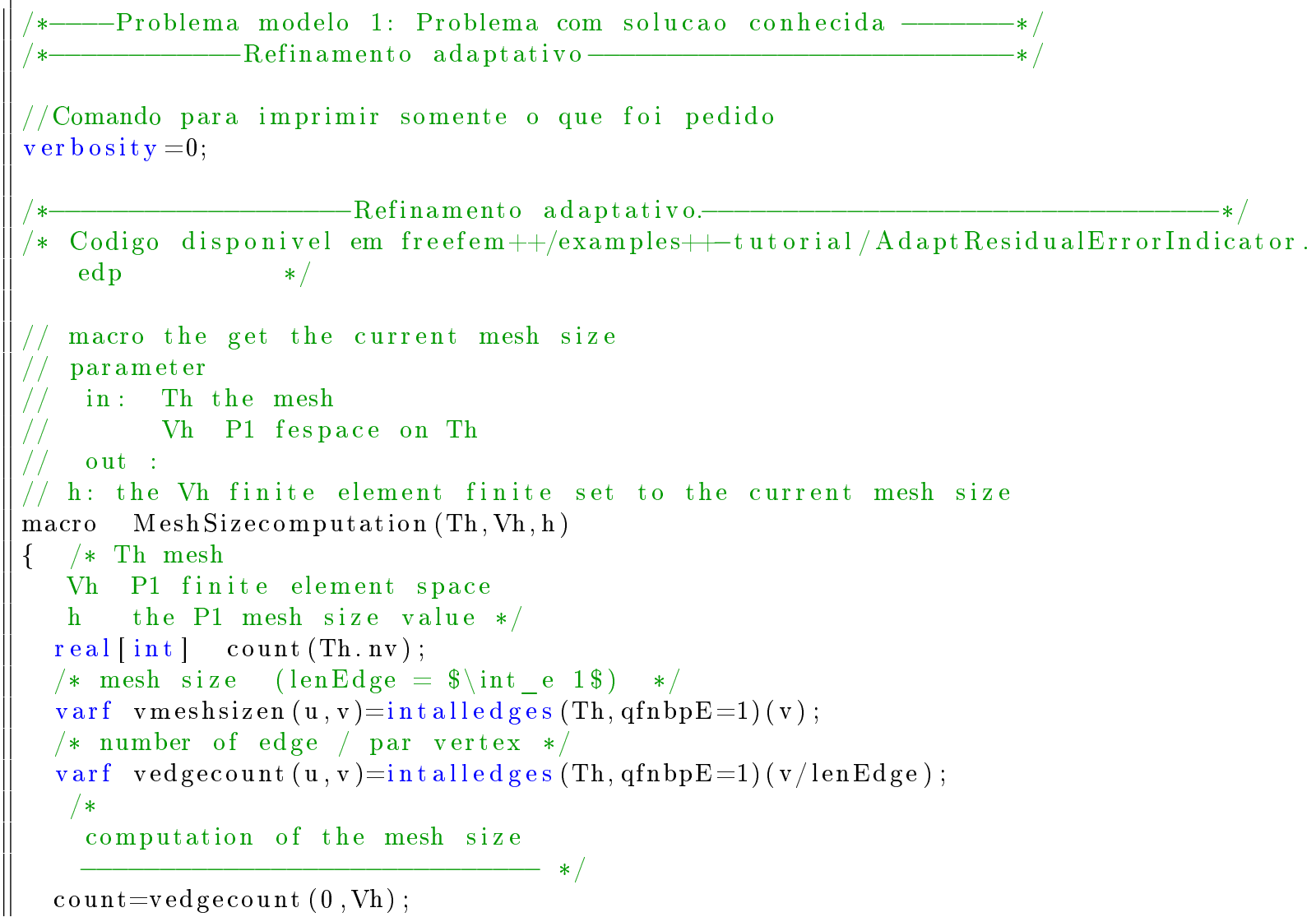




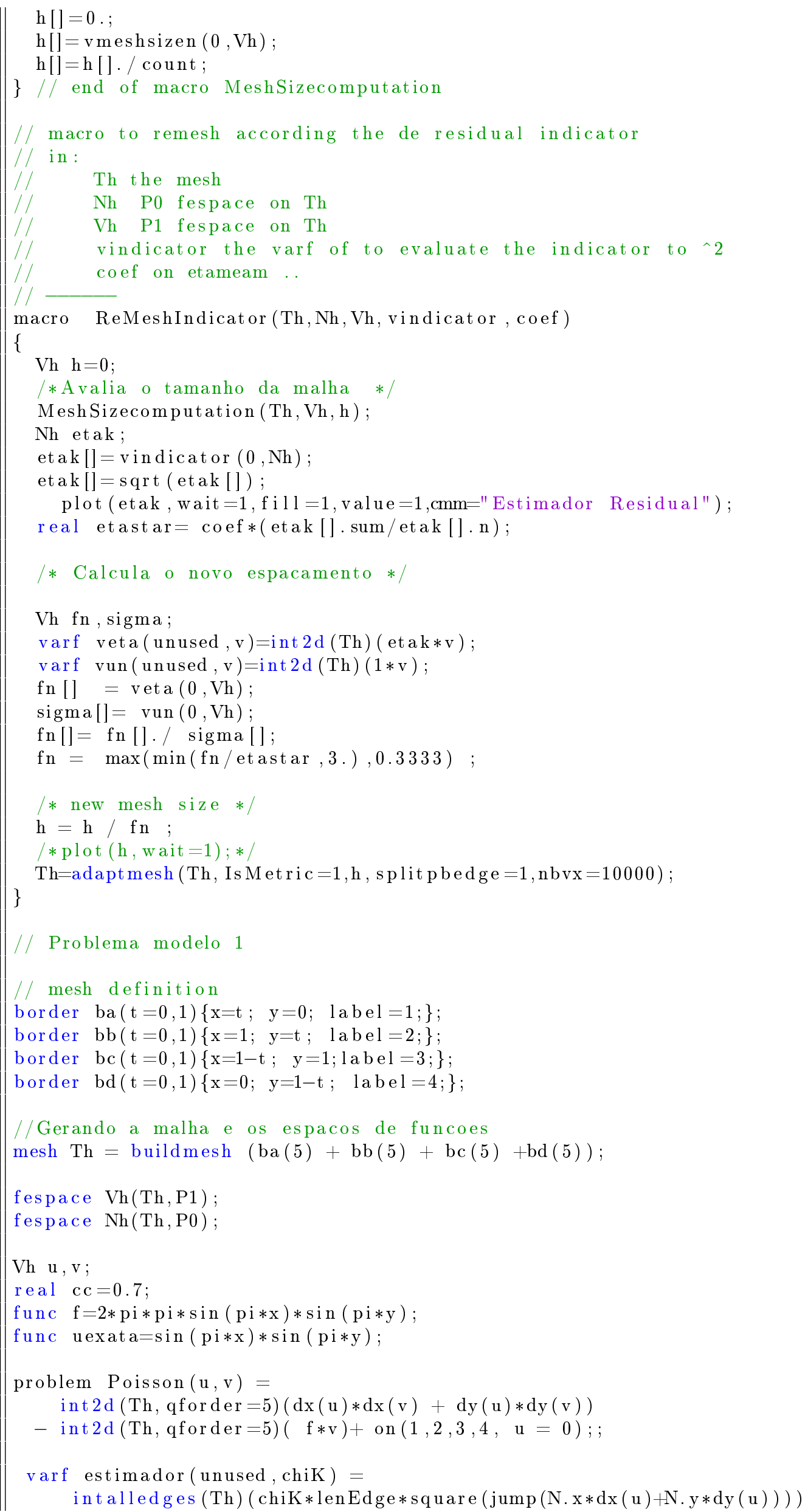




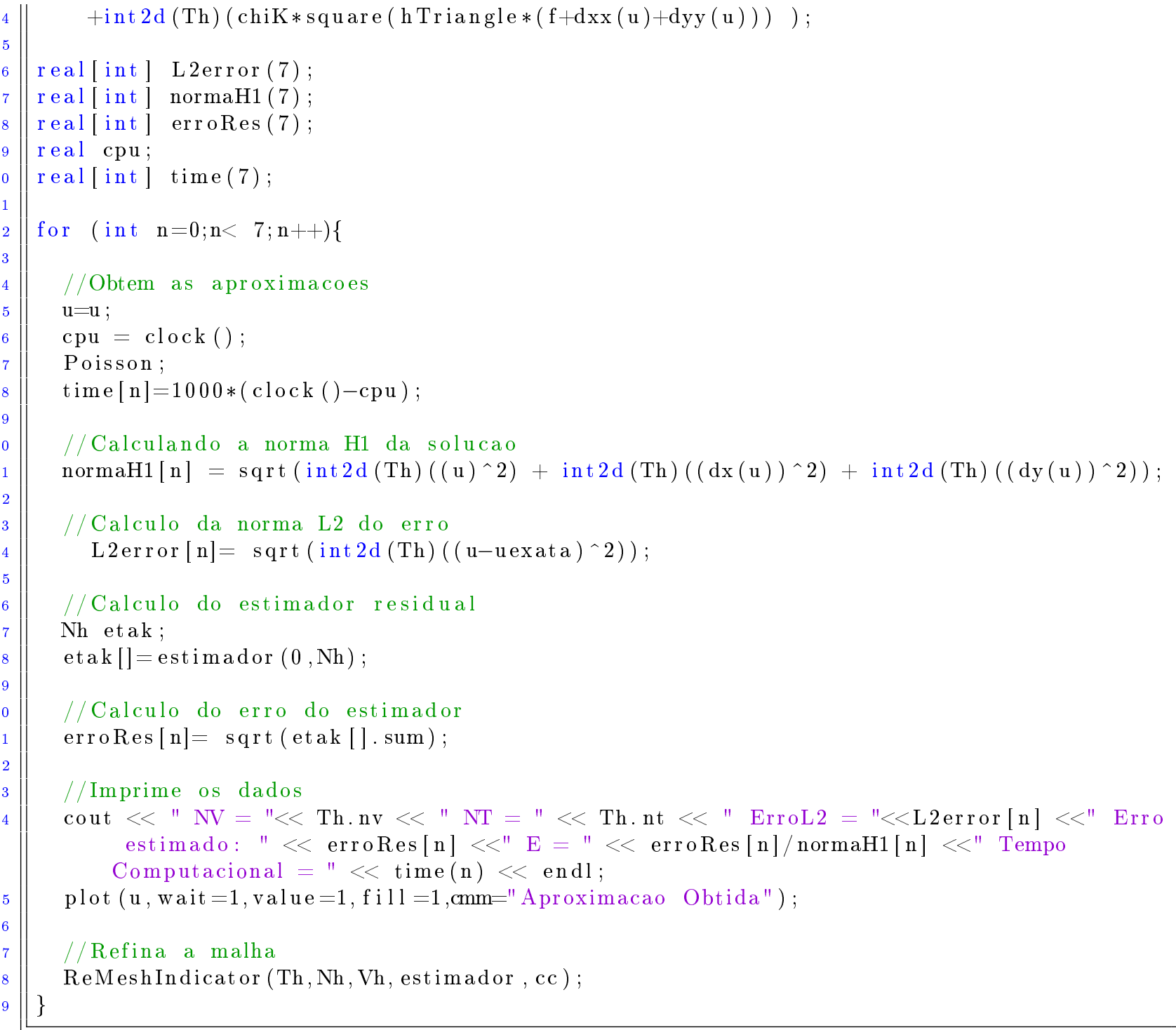

Listing B.3: testes_cap3/teste2.edp

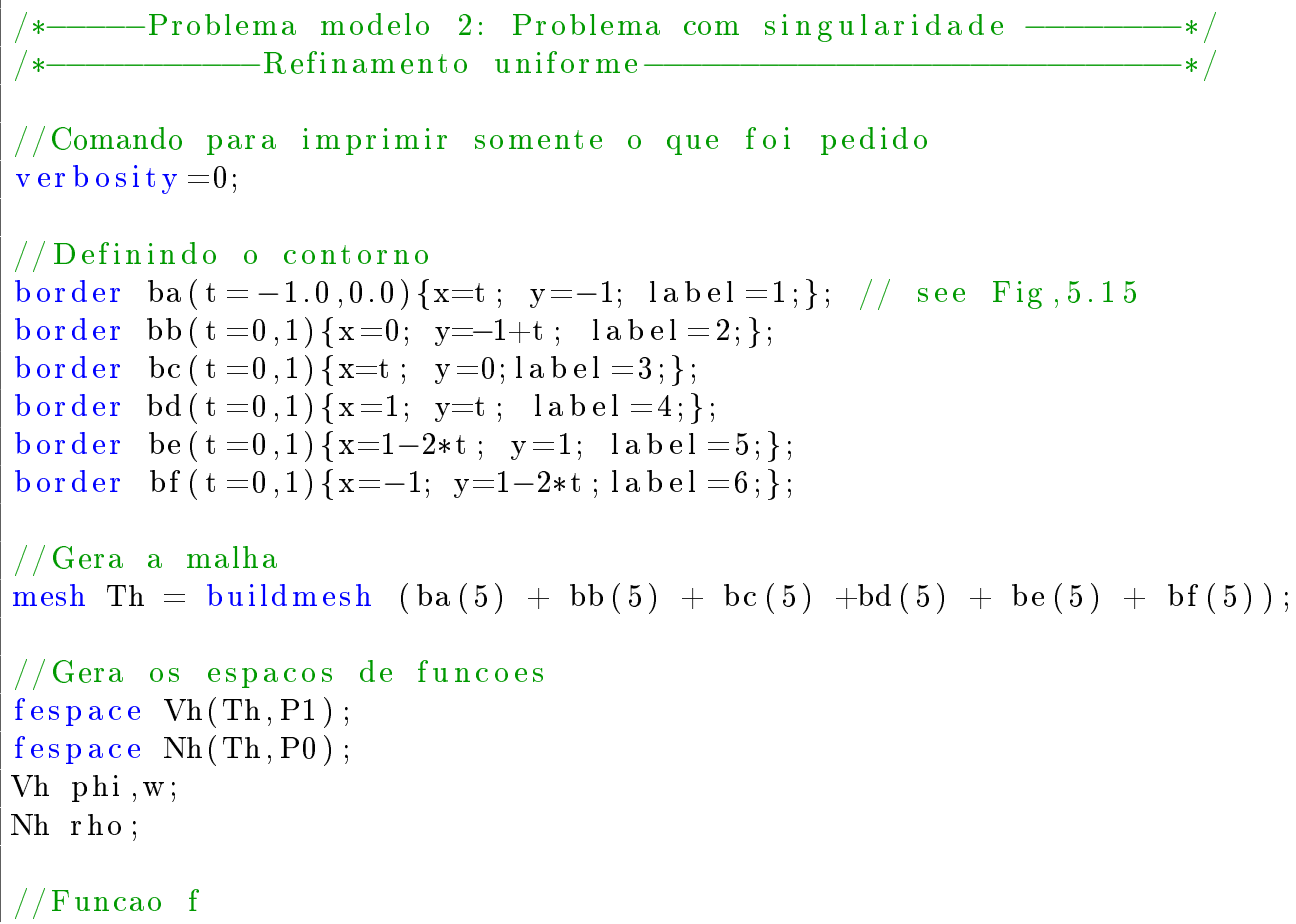




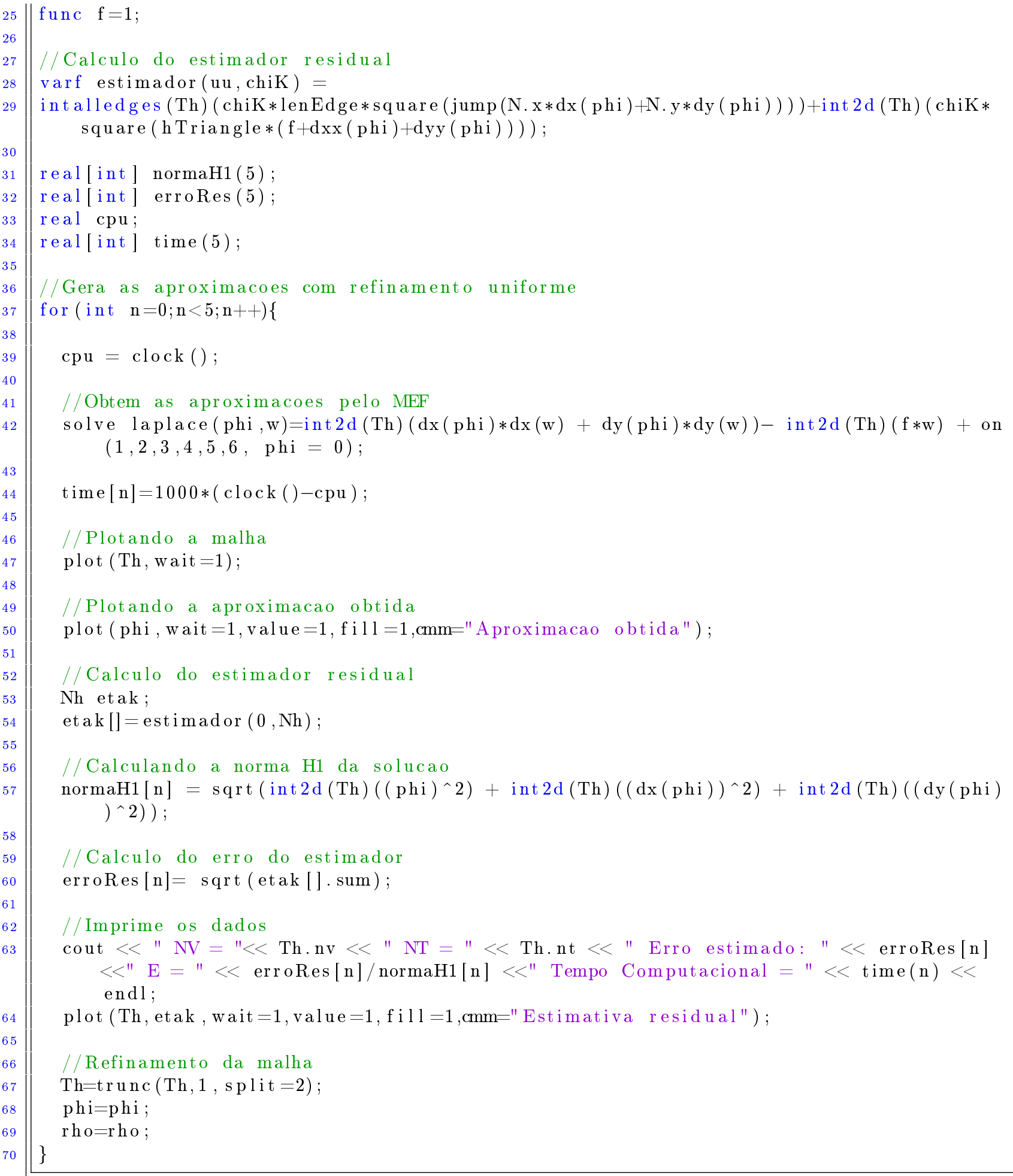

Listing B.4: testes_cap3/teste2_adapt.edp

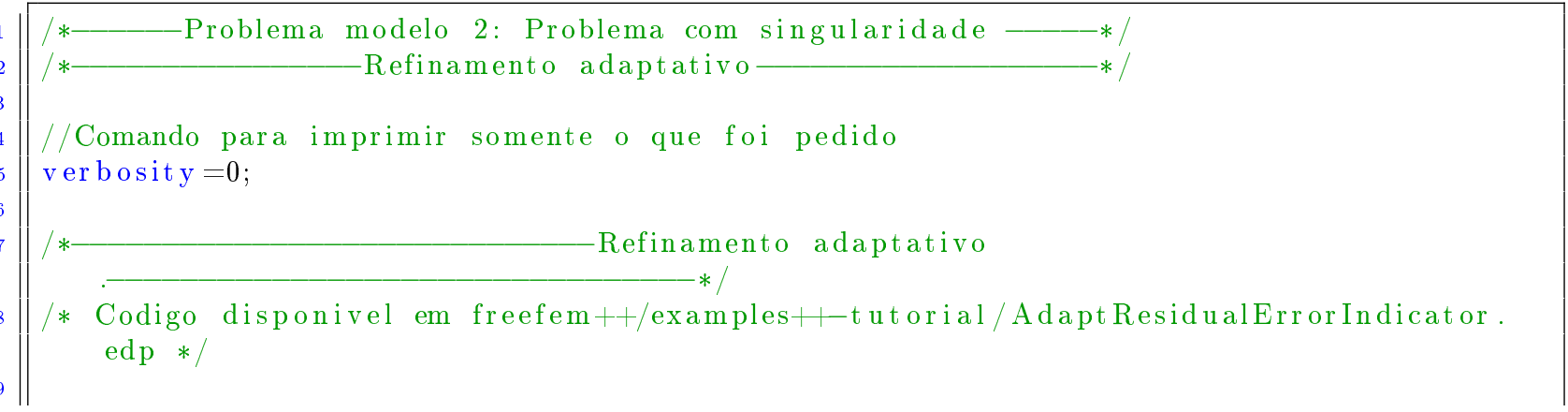




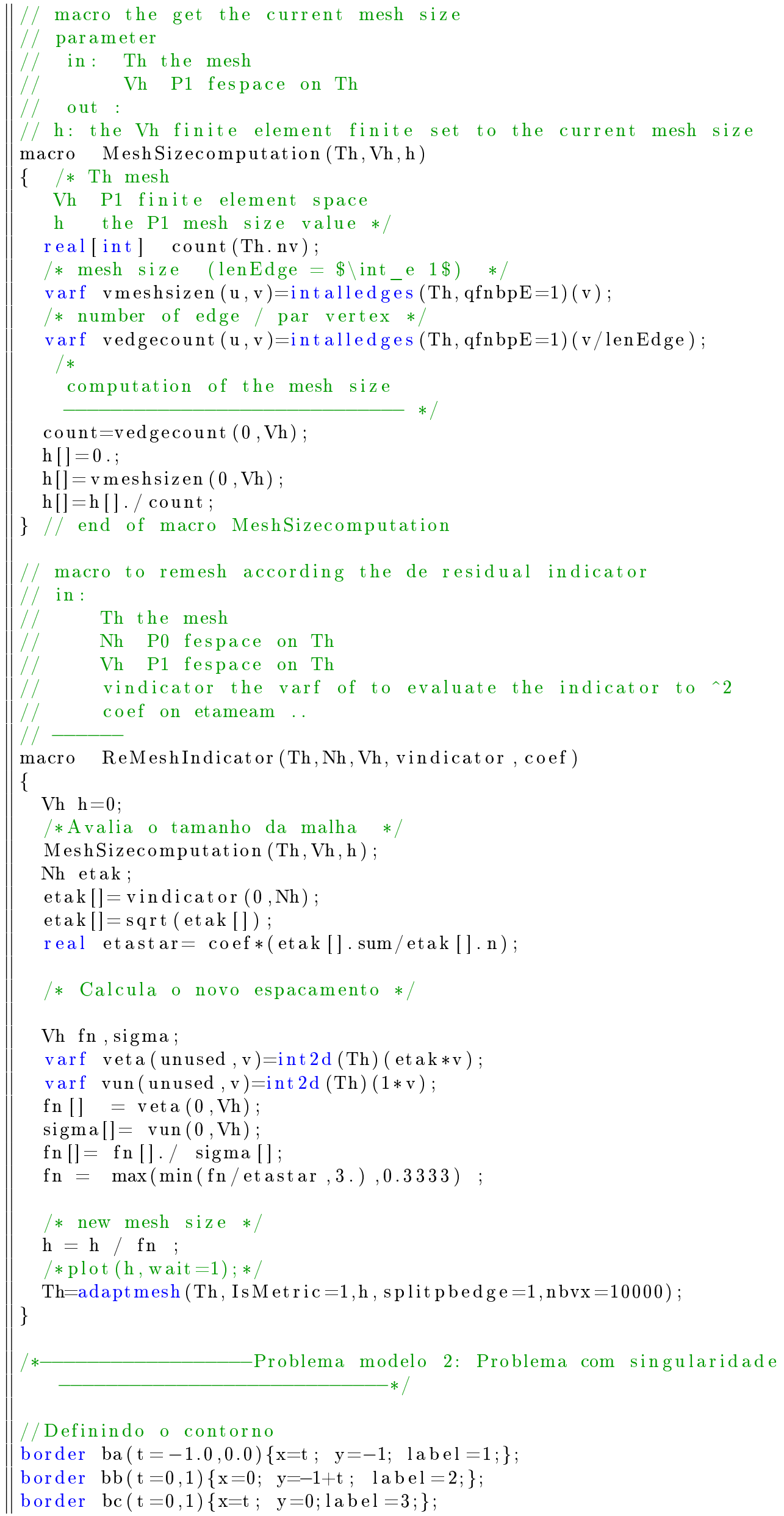




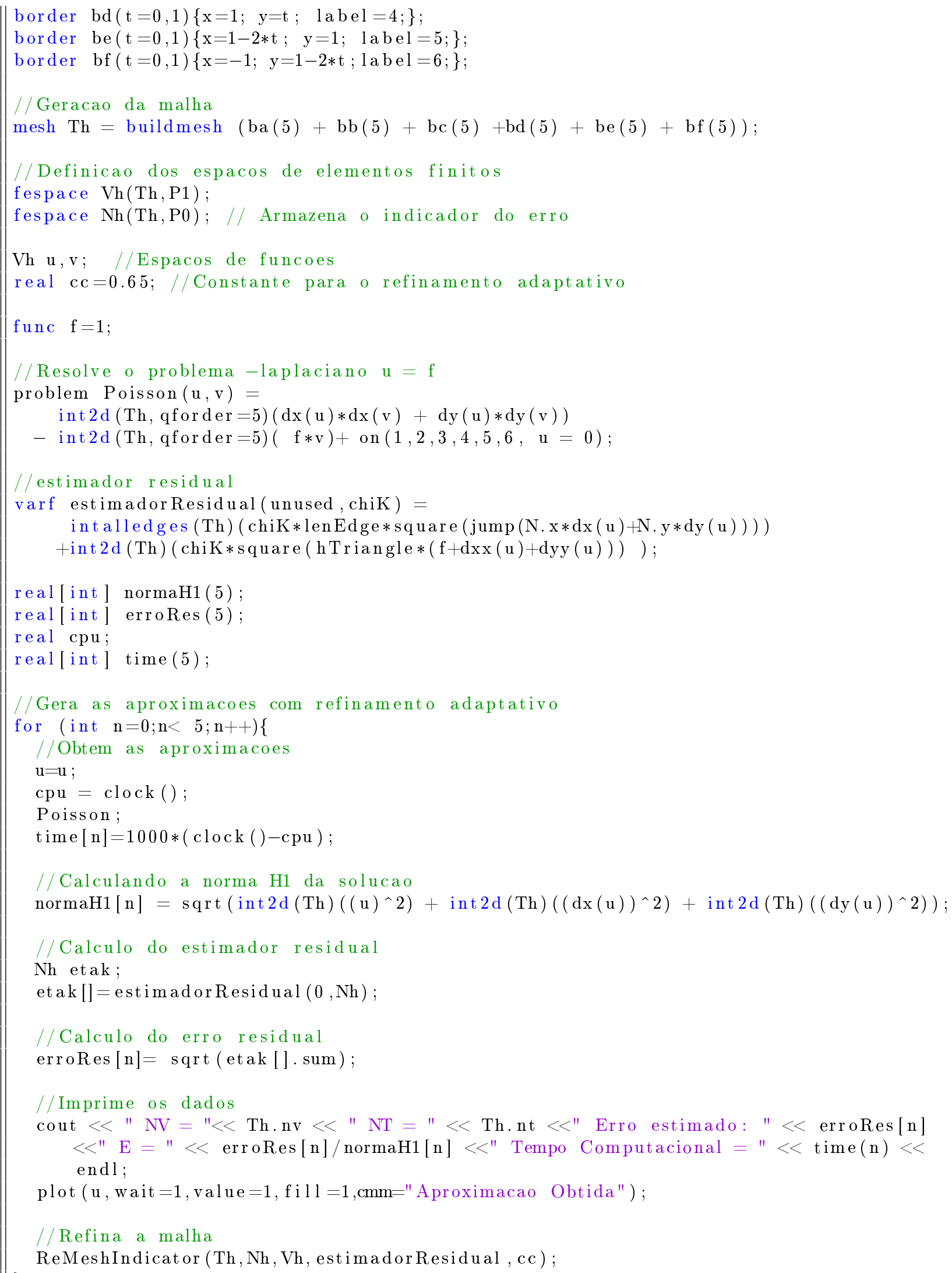

Listing B.5: testes_cap4/teste3_unif.edp

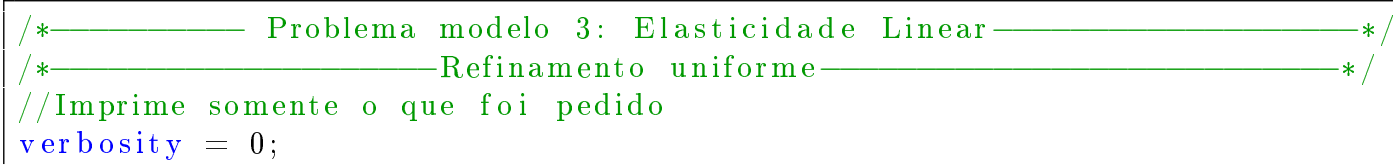




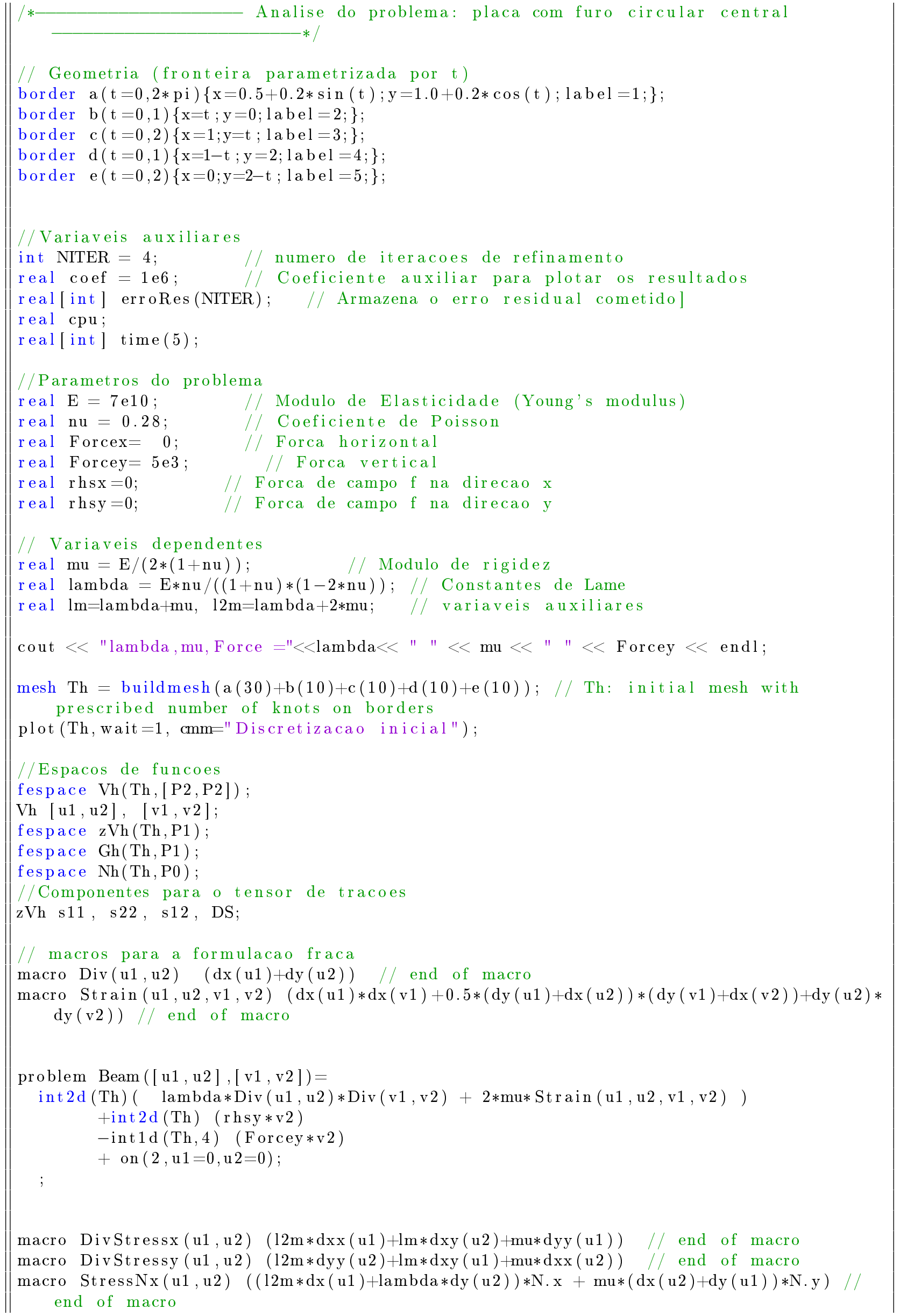




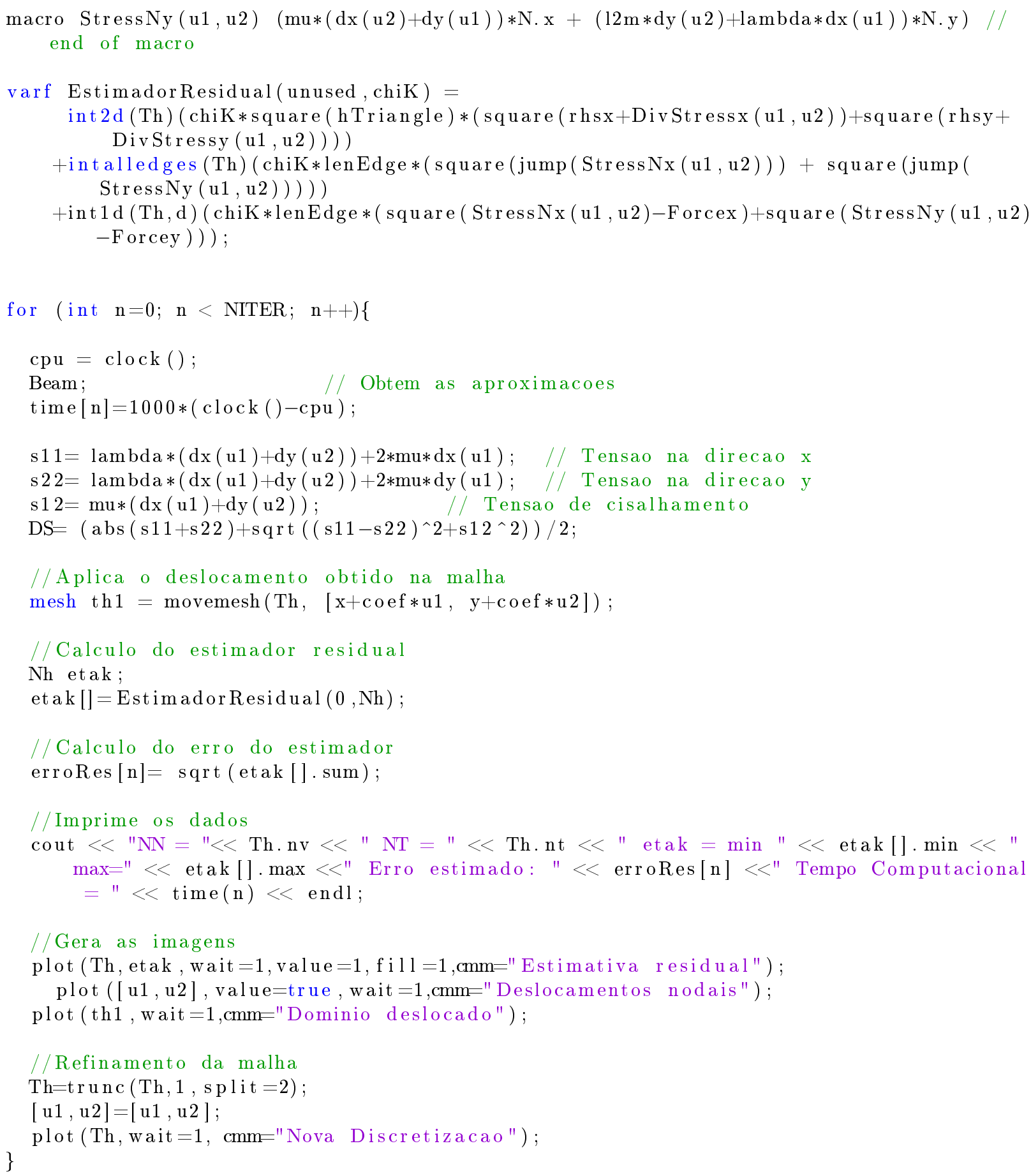

Listing B.6: testes cap4/teste3 adapt.edp

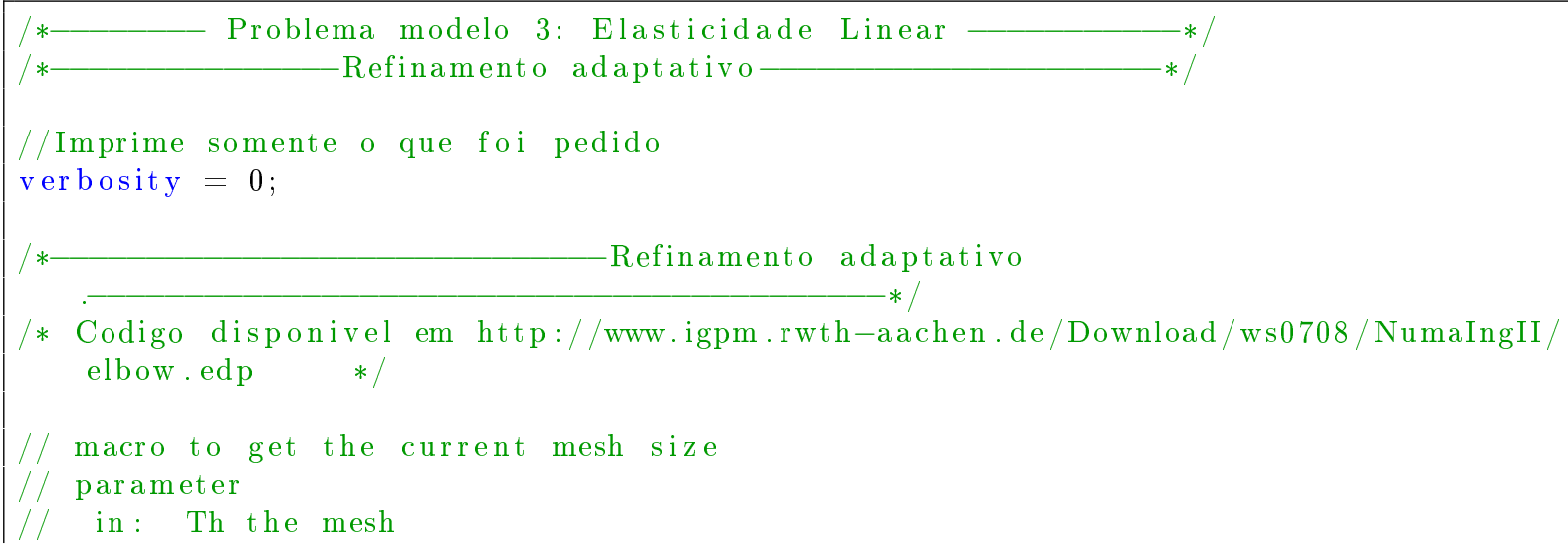




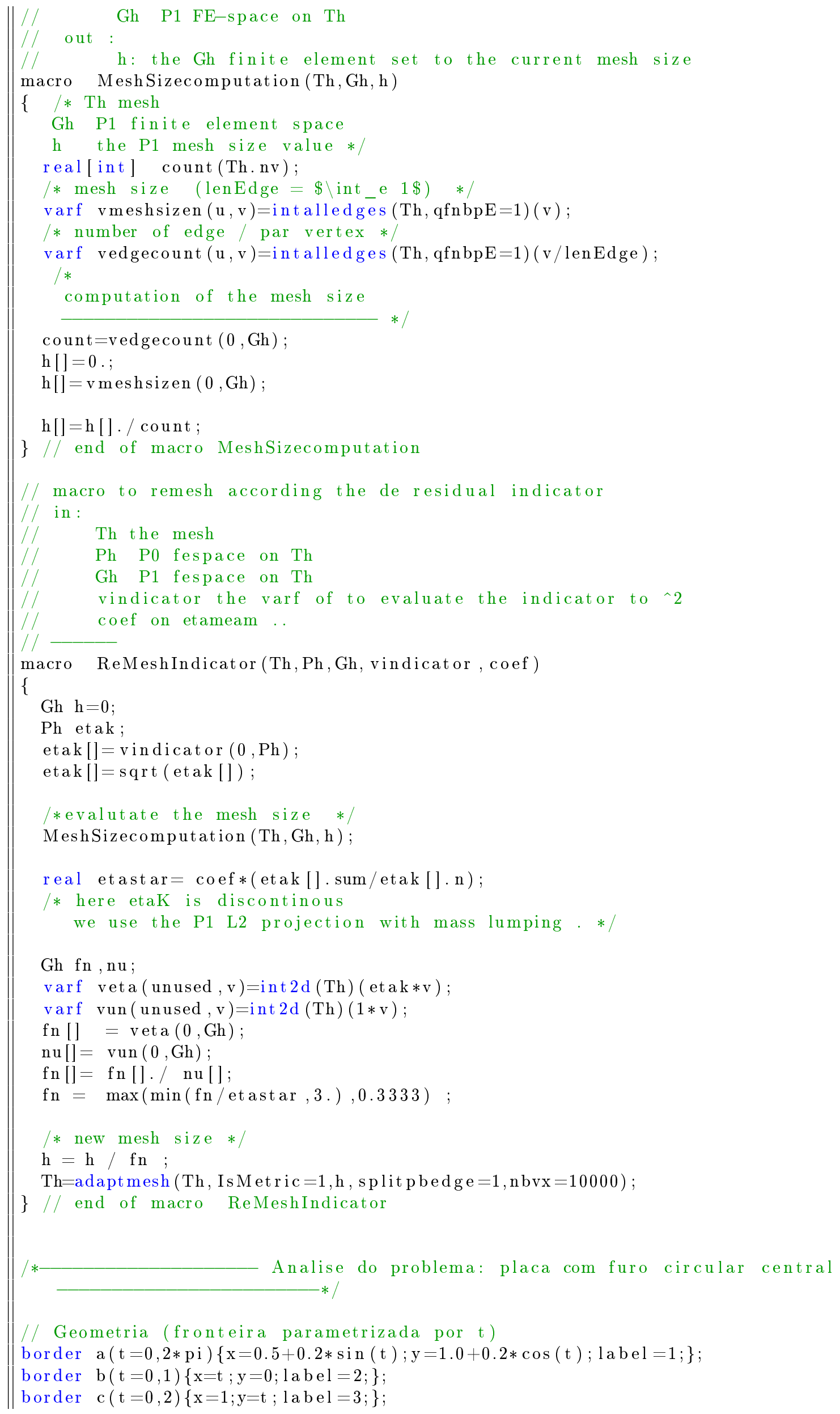




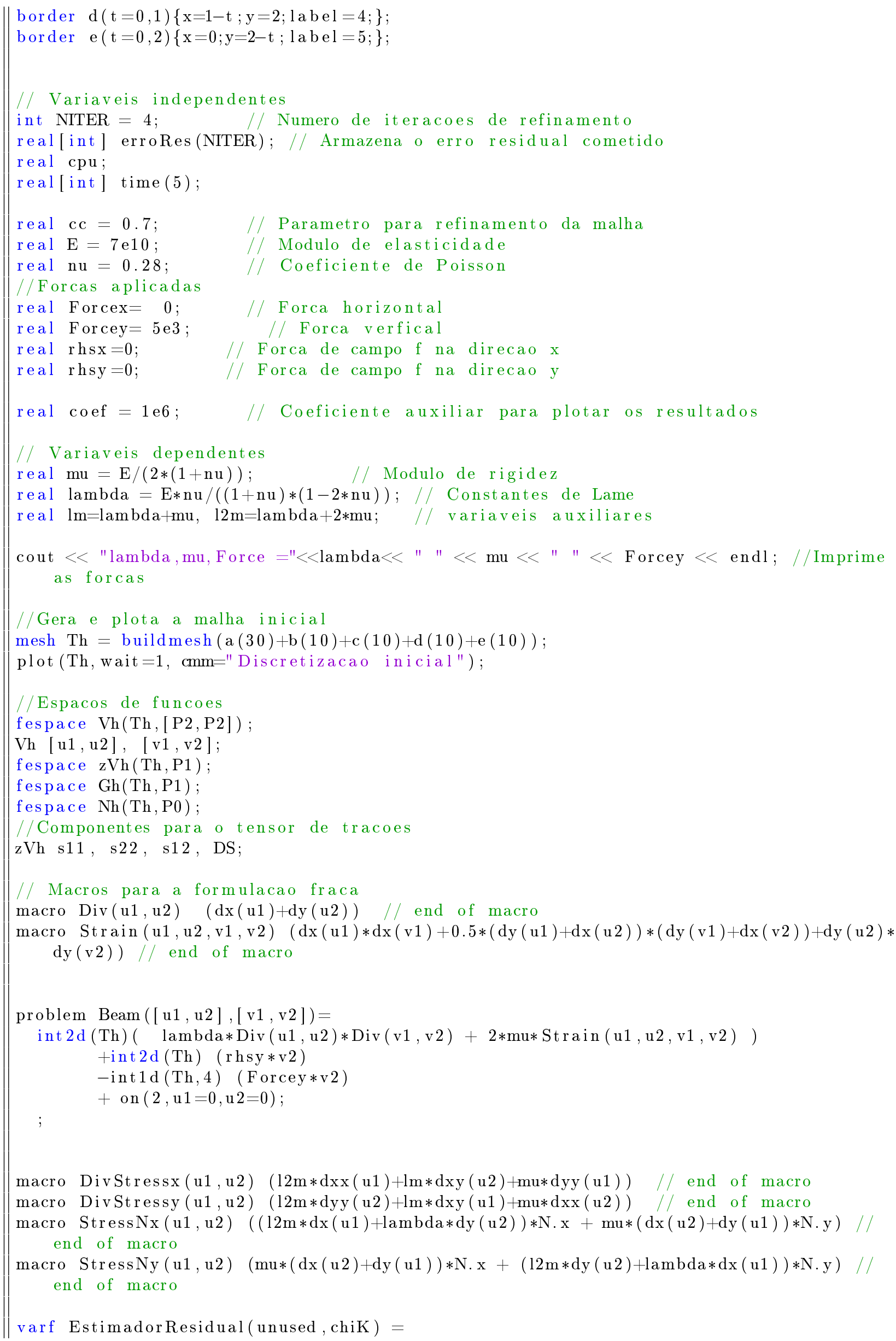




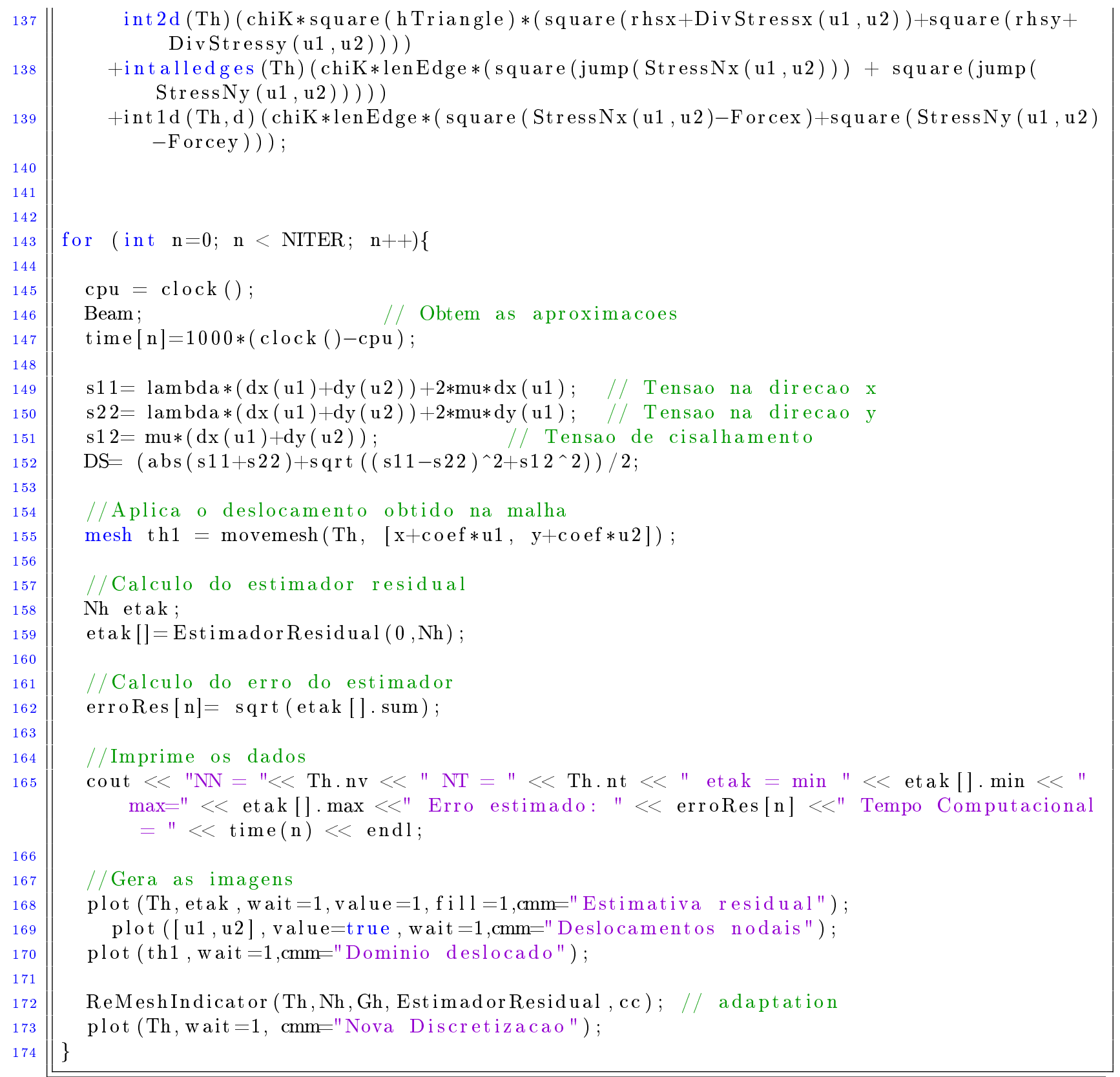




\section{Apêndice C}

\section{Figuras}

Neste apêndice encontram-se as figuras obtidas nas simulações em seus tamanhos originais.

\section{C.1 Problema de Poisson}

\section{C.1.1 Problema 3.16}

Aproximacao obtida

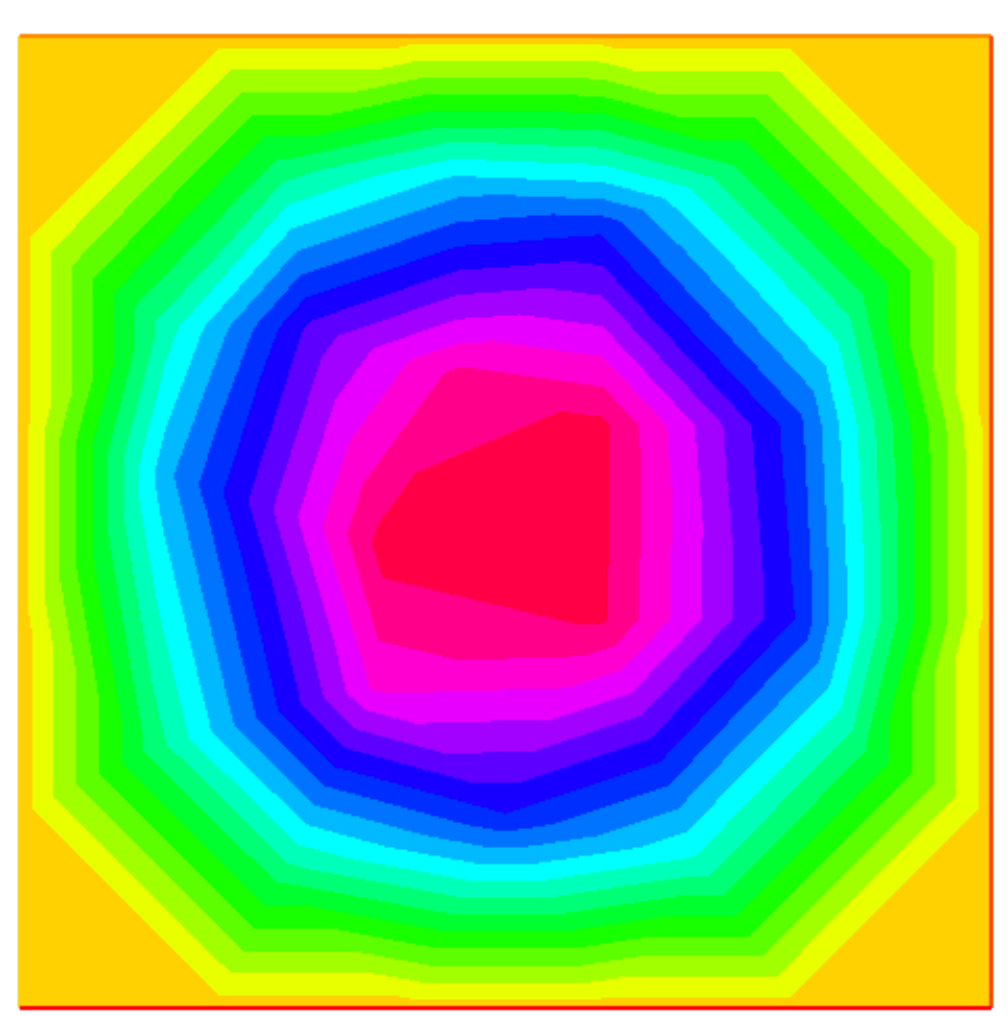

Figura C.1: Aproximação obtida com elementos lineares - Fig (3.2a) 
Estimativa residual

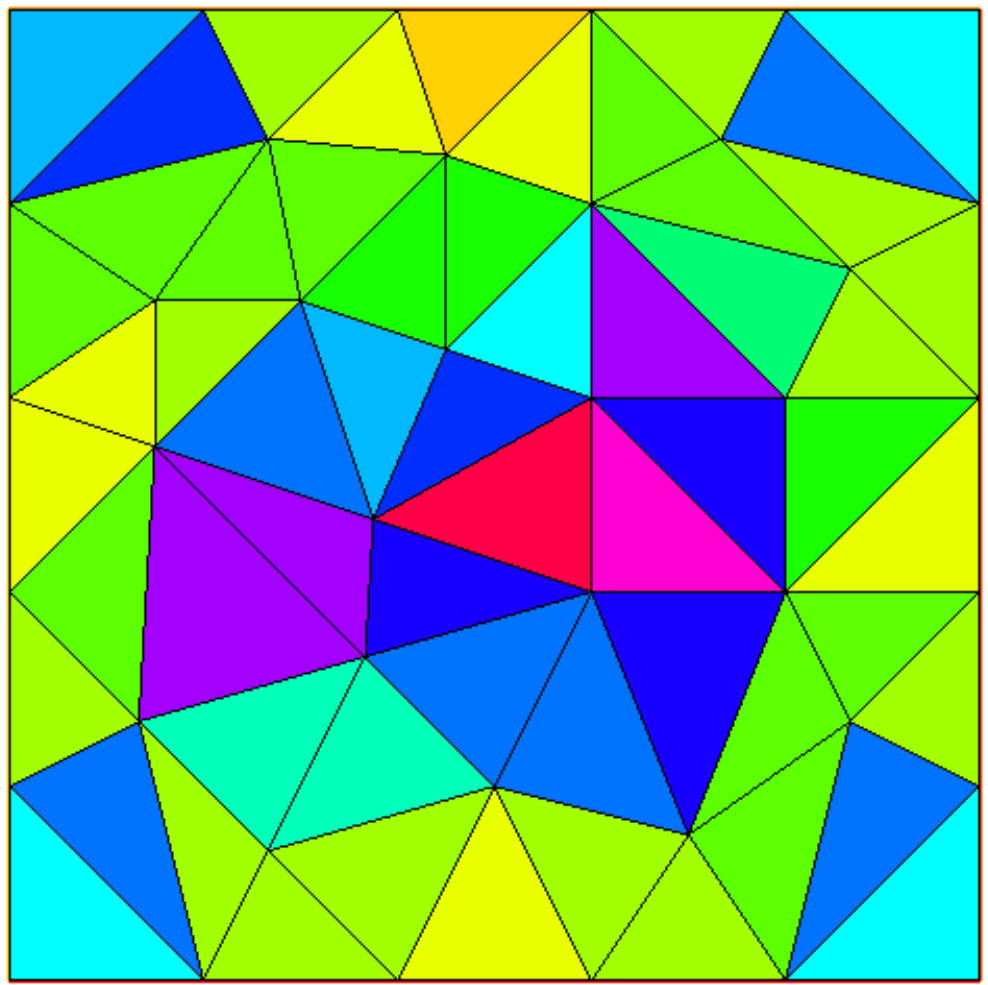

IsoValue

0.0389148

0.11357

0.15091

0.225573

0.262904

0.337568

0.374899
-0.412231

.449562

0.561557

0.598889

0.63622

710884

Figura C.2: Residuo obtido na aproximação - Fig (3.2b)

Aproximacao obtida
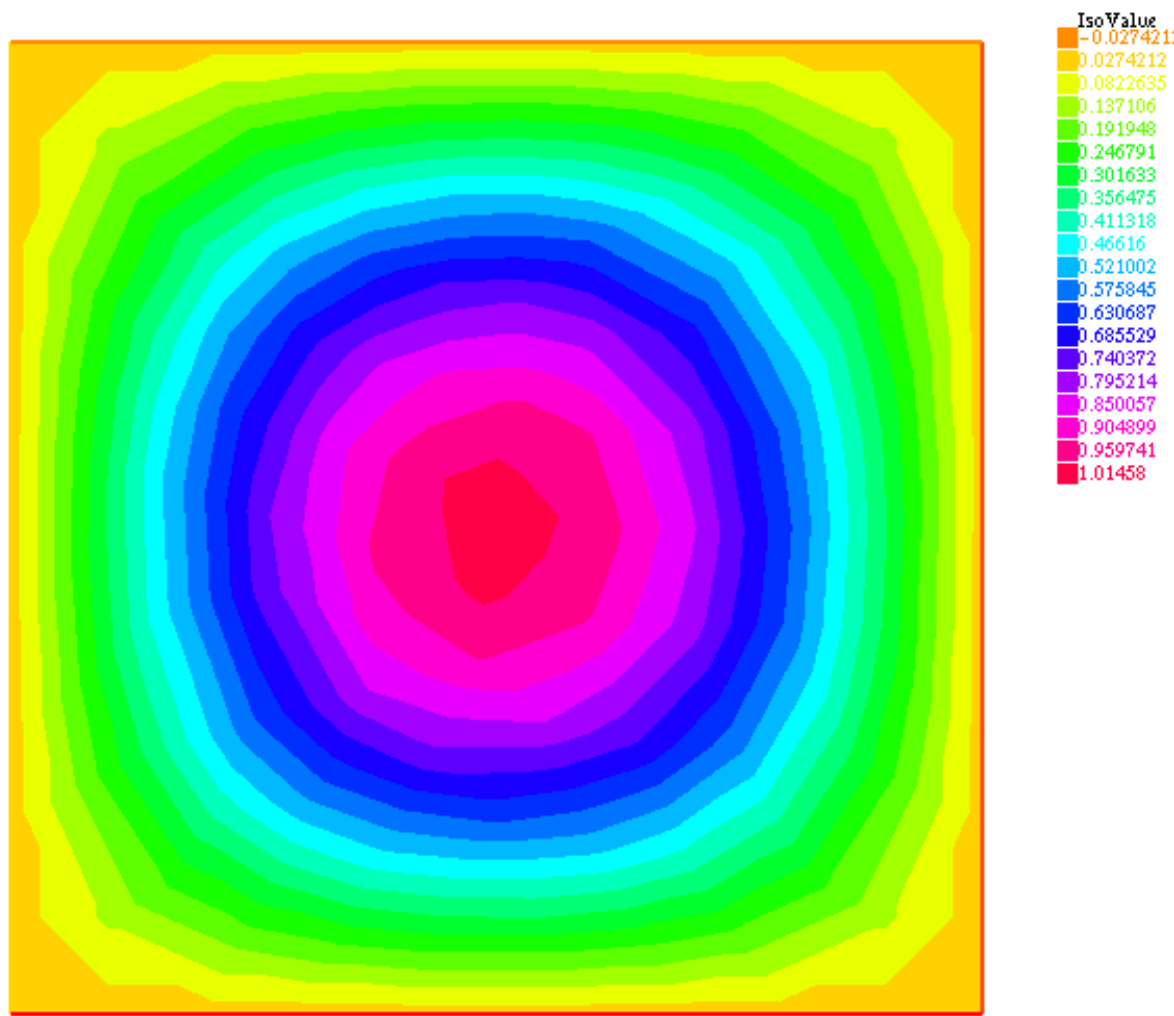

Figura C.3: Aproximação obtida na primeira iteração do refinamento uniforme - Fig (3.3a) 


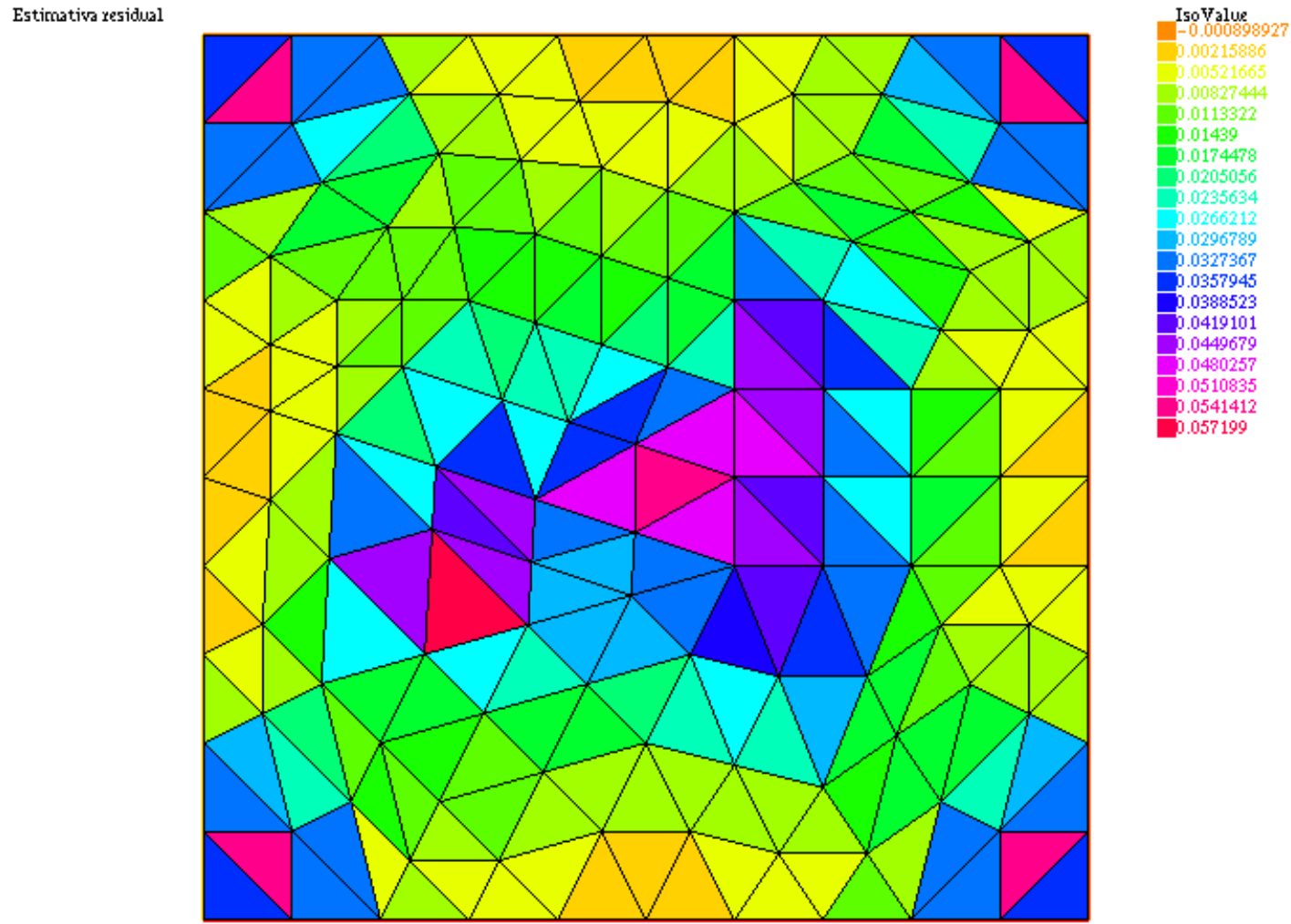

Figura C.4: Residuo obtido na aproximação da malha gerada pela primeira iteração do refinamento uniforme - Fig (3.3b)

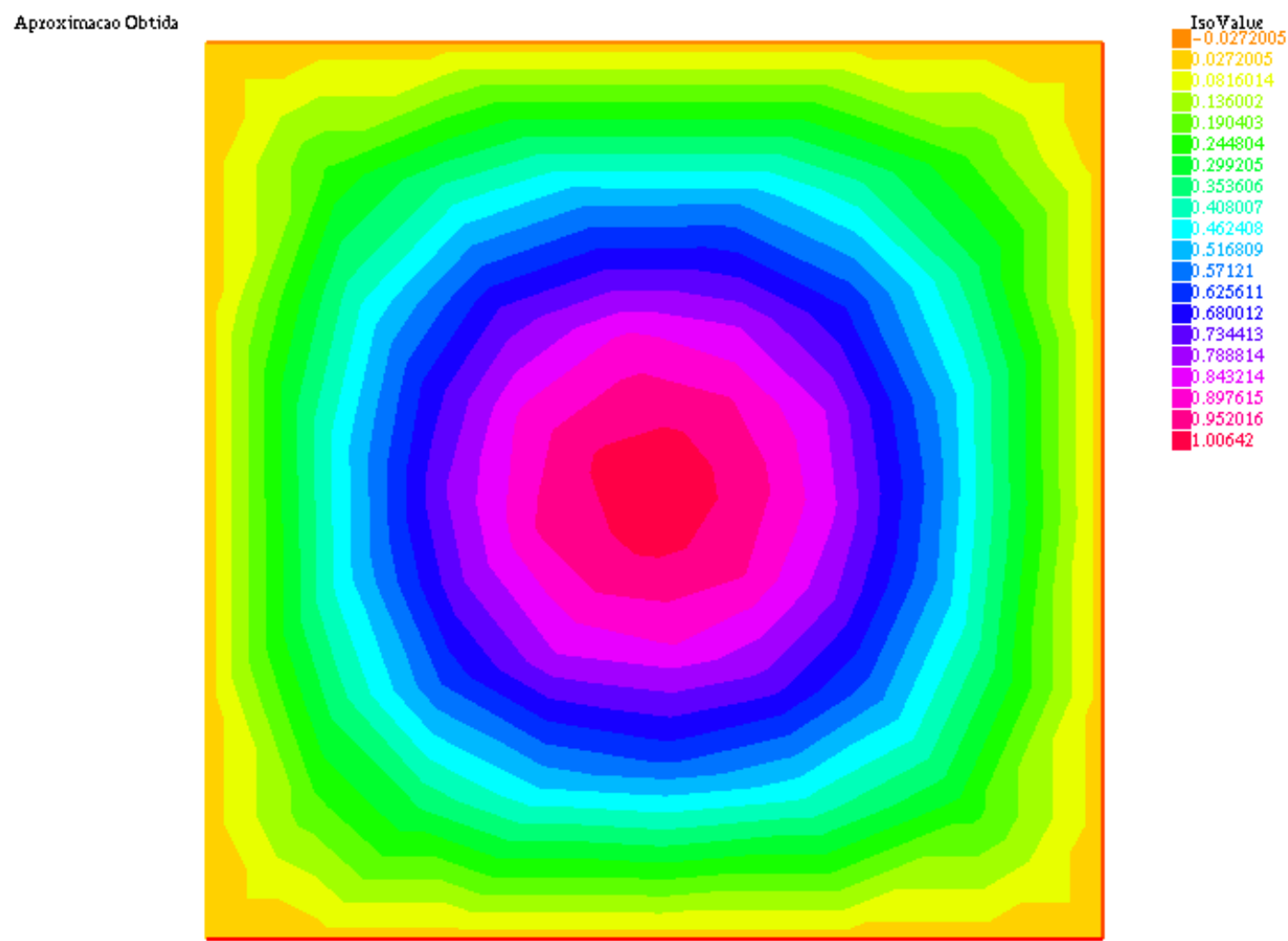

Figura C.5: Aproximação obtida na primeira iteração do refinamento adaptativo - Fig (3.4a) 
Estimador Residual
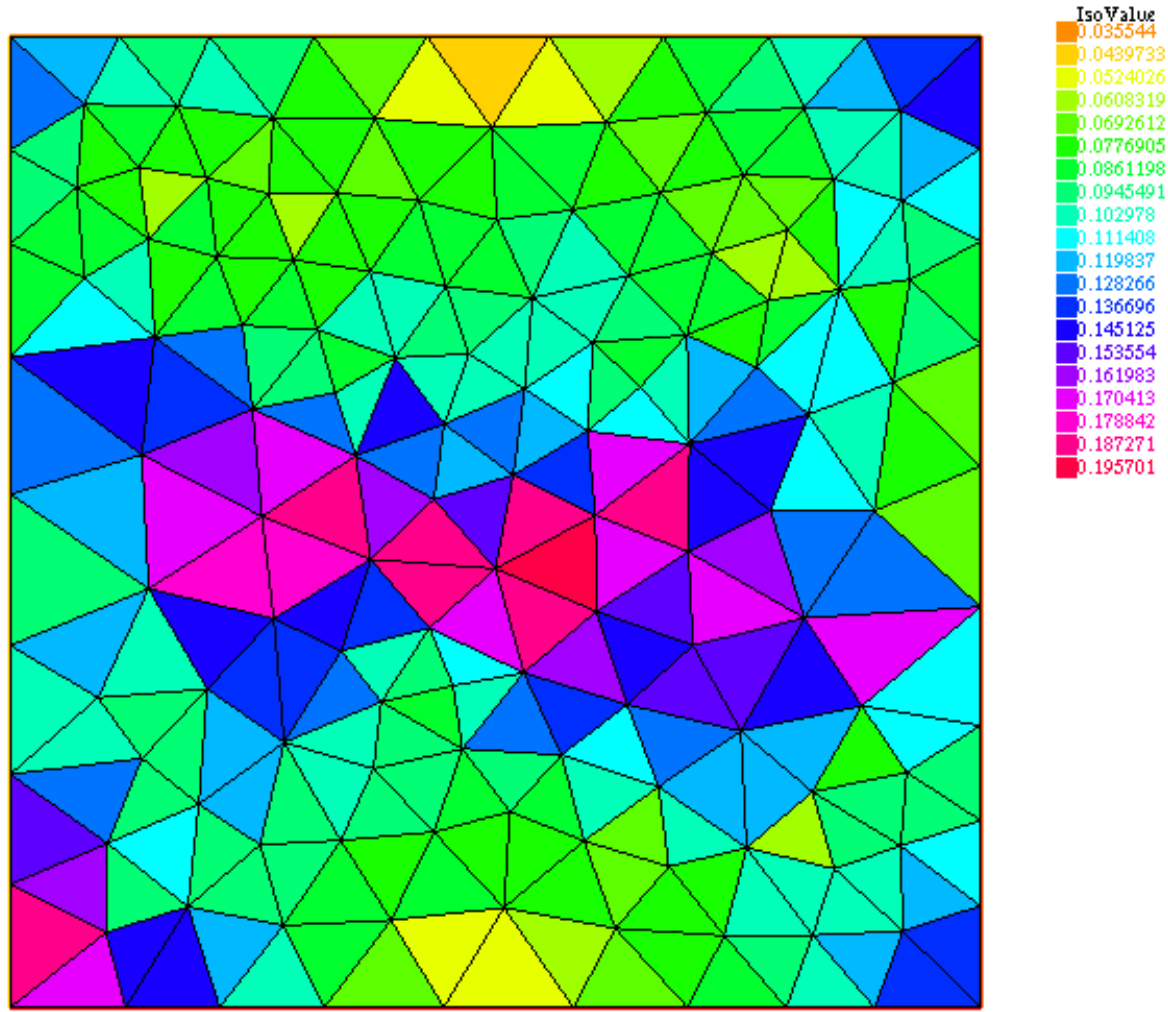

Figura C.6: Residuo obtido na aproximação da malha gerada pela primeira iteração do refinamento adaptativo - Fig (3.4b)

Aproximacao obtida
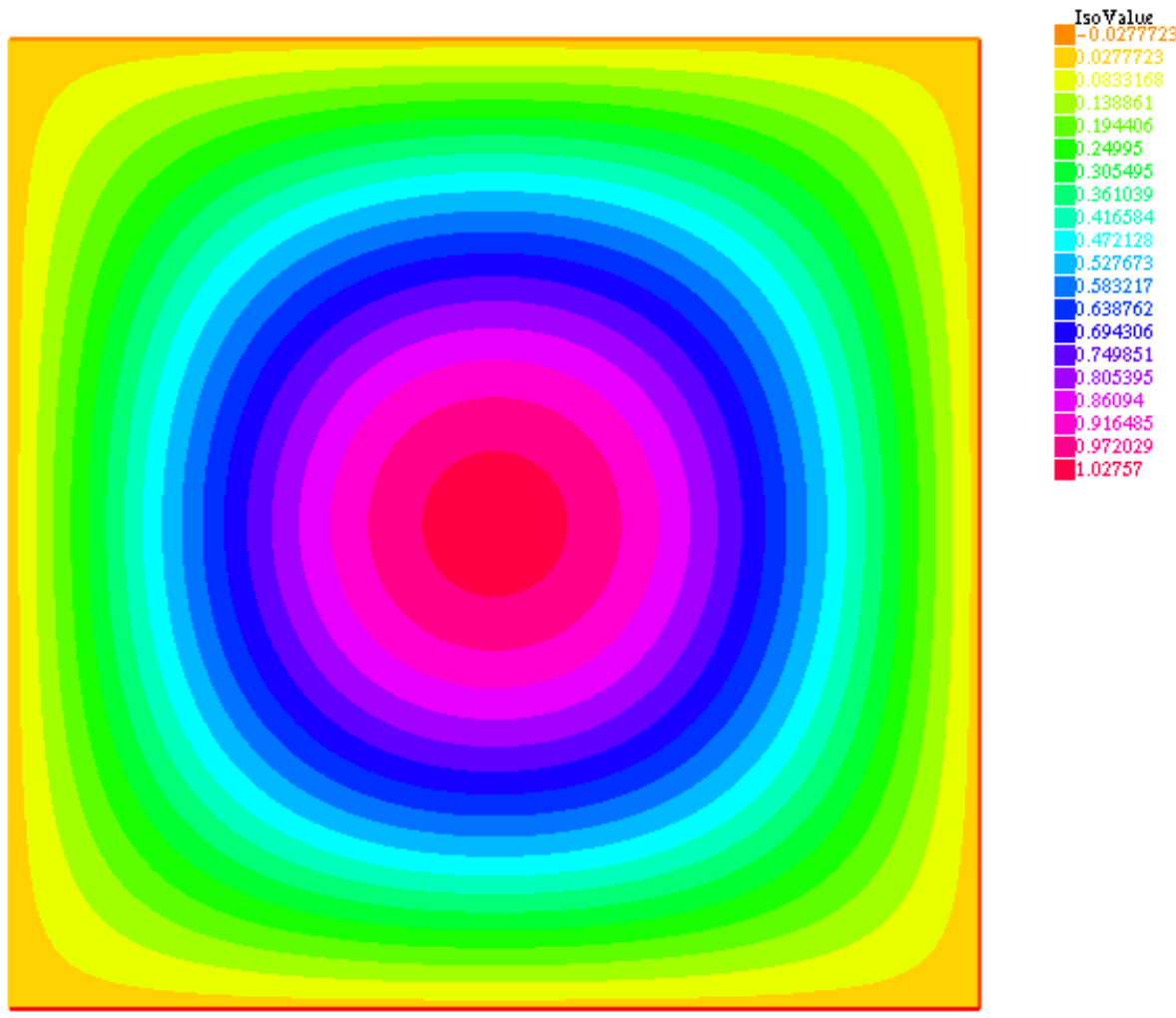

Figura C.7: Aproximação obtida na quarta iteração do refinamento uniforme - Fig (3.5a) 


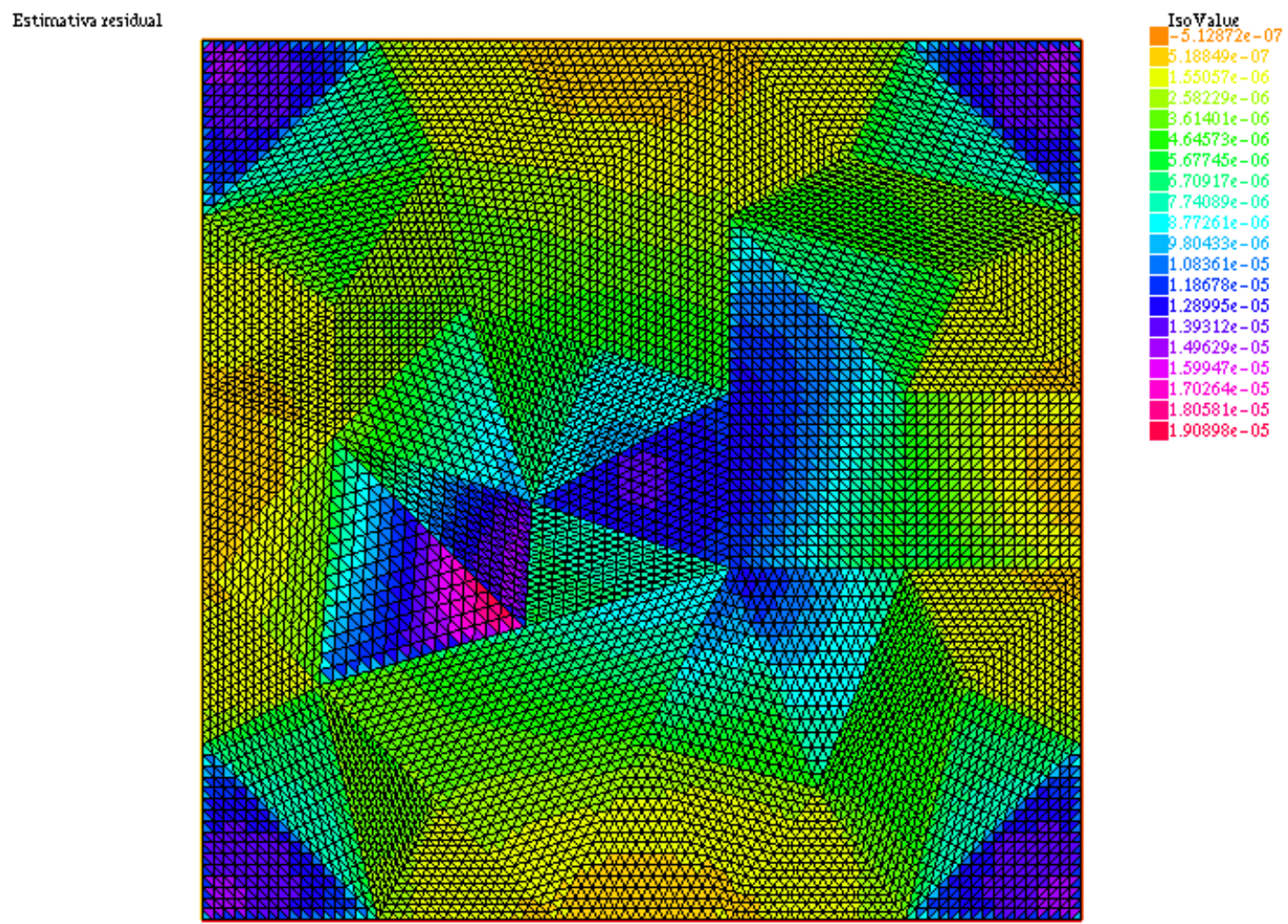

Figura C.8: Residuo obtido na aproximação da malha gerada pela quarta iteração do refinamento uniforme - Fig (3.5b)

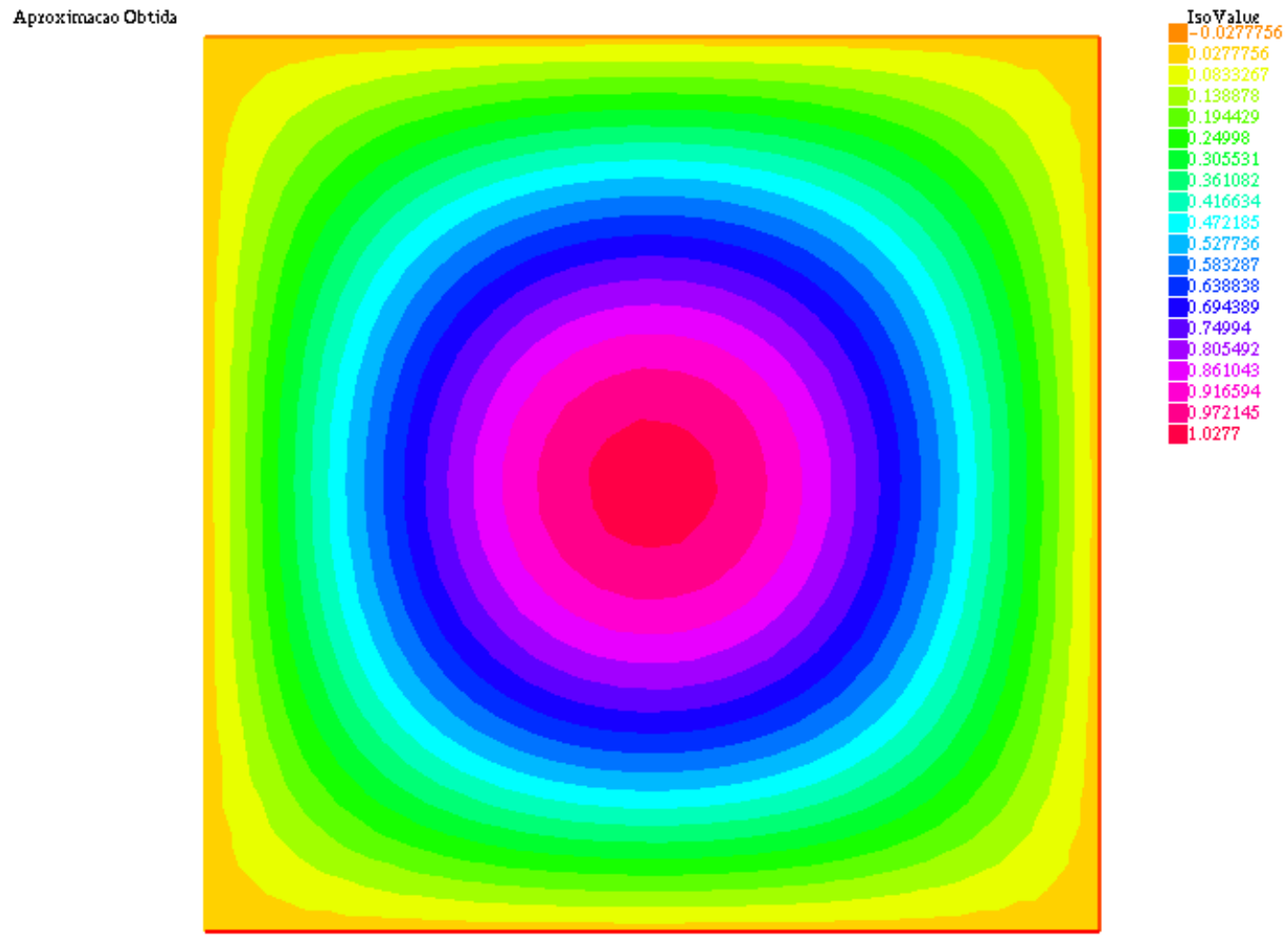

Figura C.9: Aproximação obtida na quarta iteração do refinamento adaptativo - Fig (3.6a) 
Estimador Residual
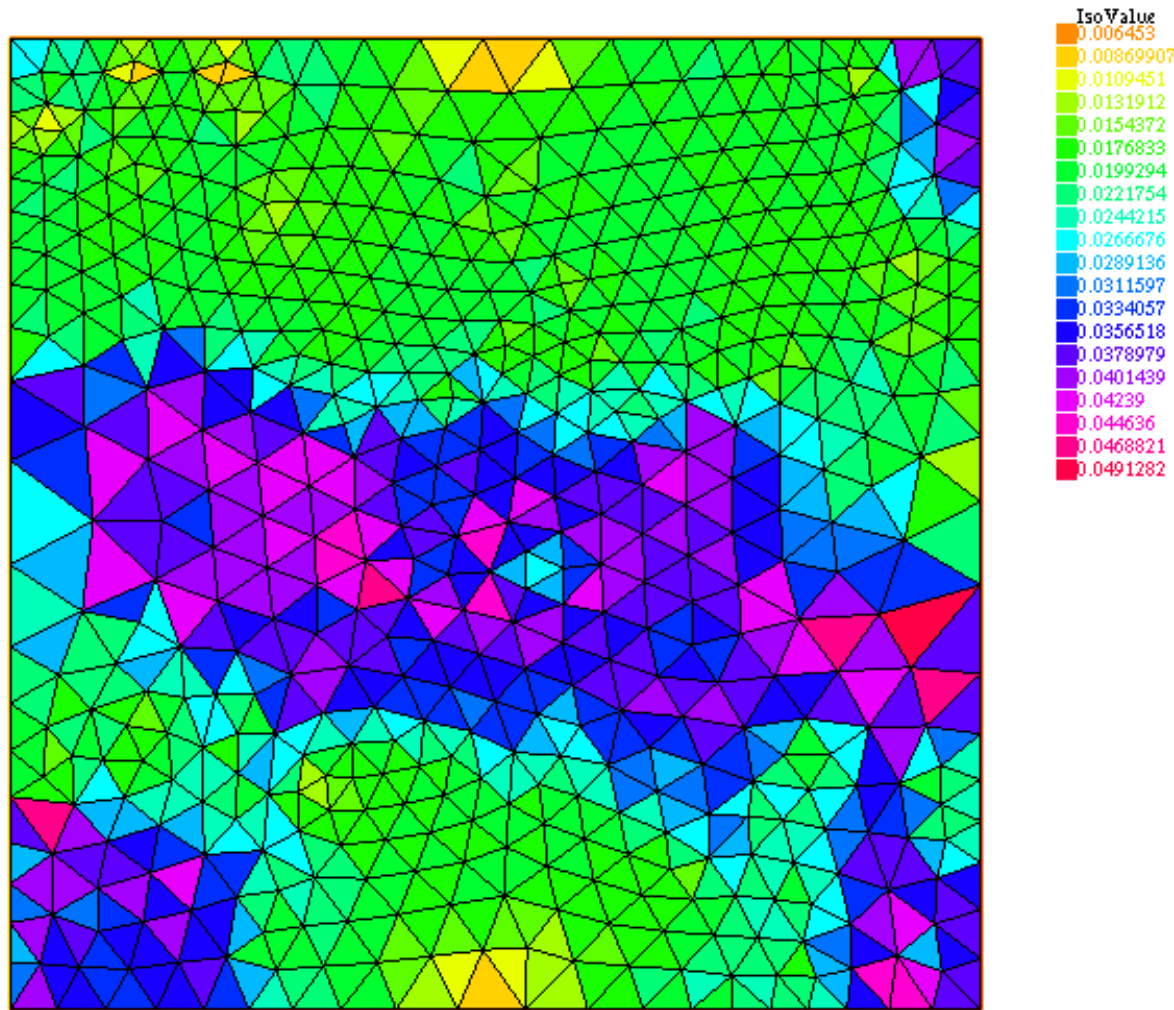

Figura C.10: Residuo obtido na aproximação da malha gerada pela quarta iteração do refinamento adaptativo - Fig (3.6b)

Estimador Residual
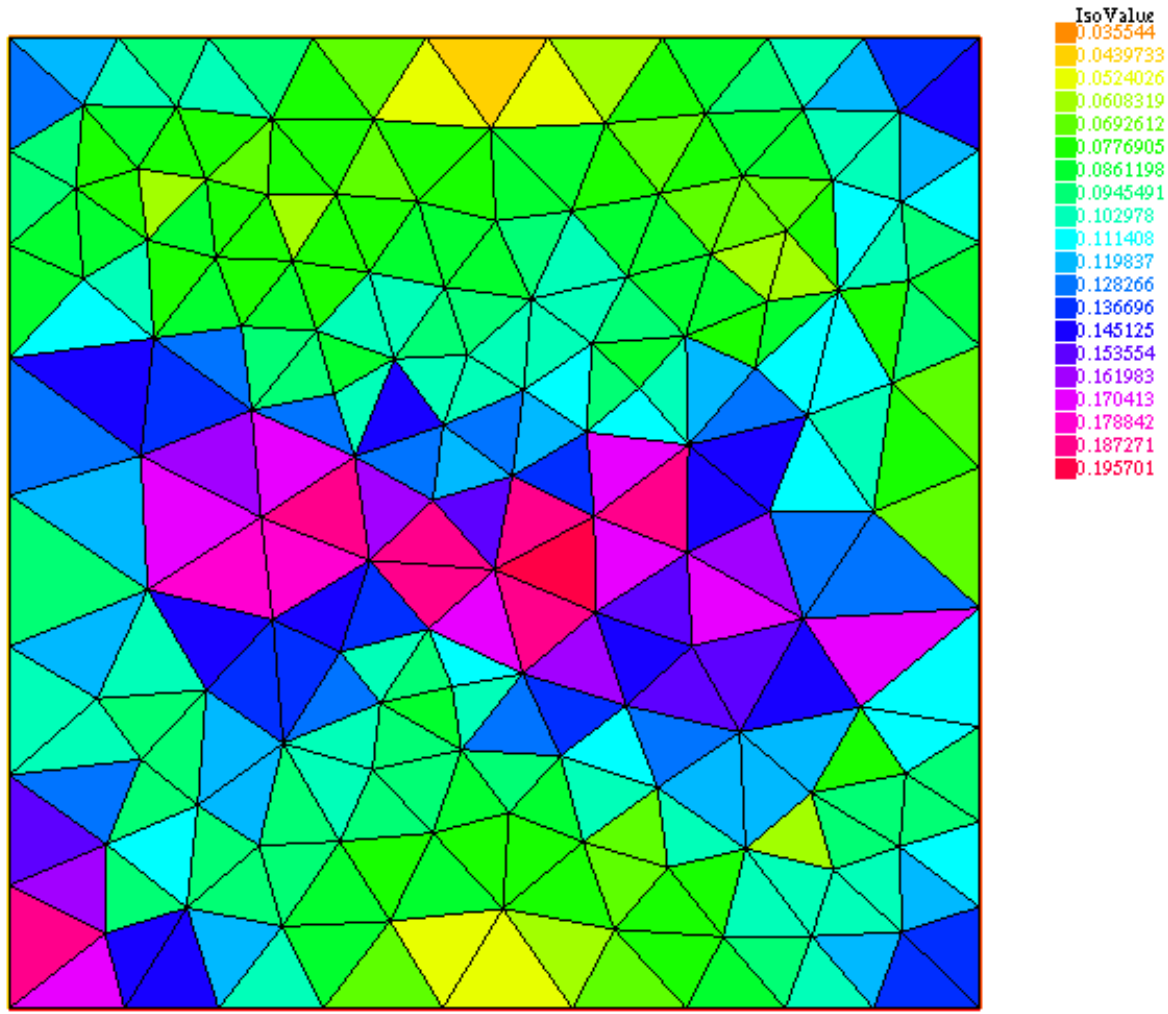

Figura C.11: Estimador residual obtido na primeira iteração do refinamento adaptativo - Fig (3.7a) 

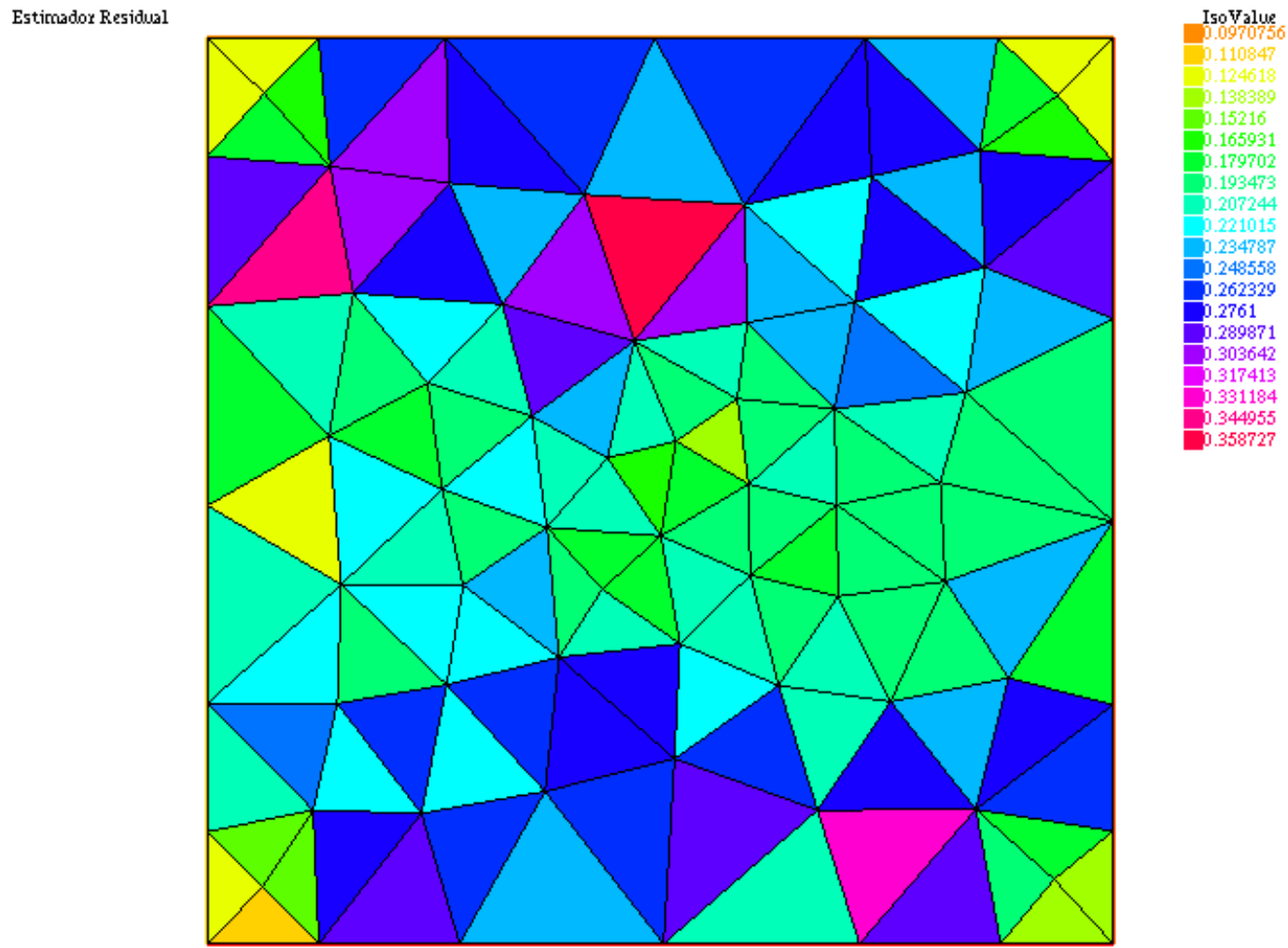

Figura C.12: Estimador residual obtido na segunda iteração do refinamento adaptativo - Fig (3.7b)

Estimador Residual

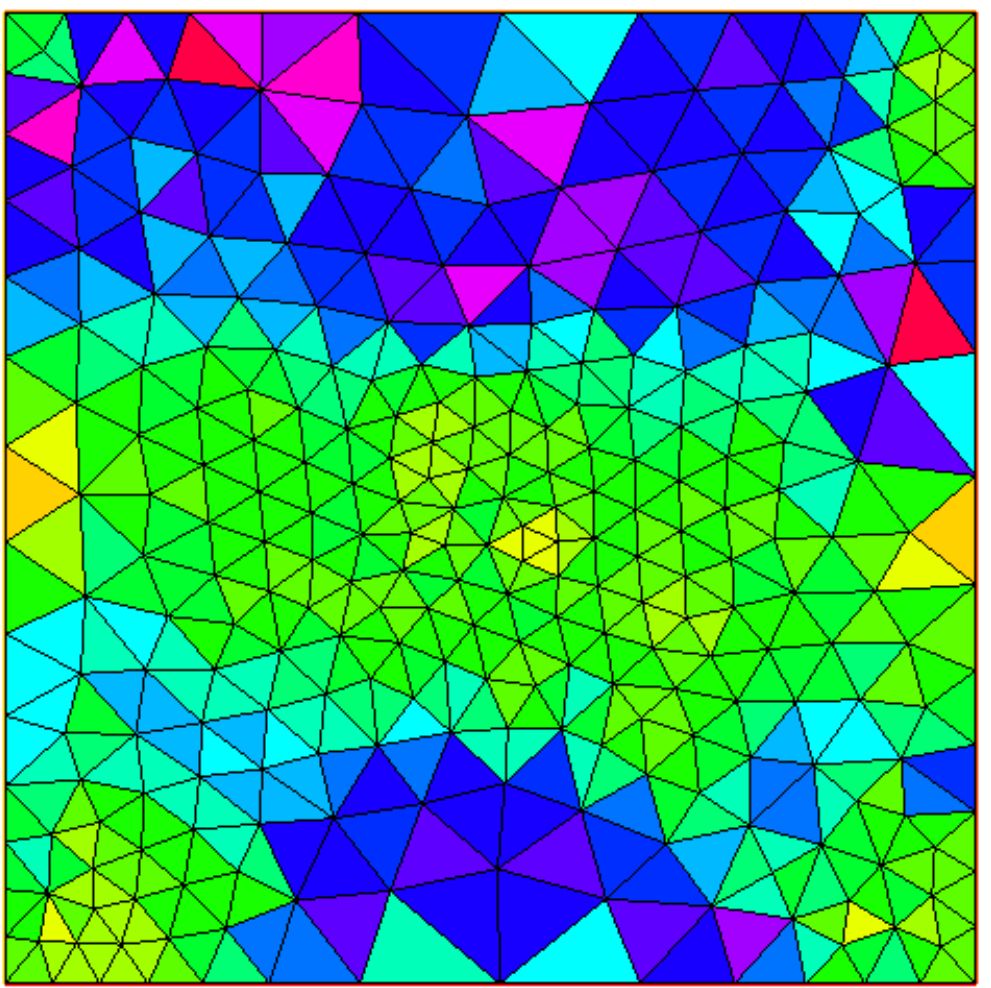

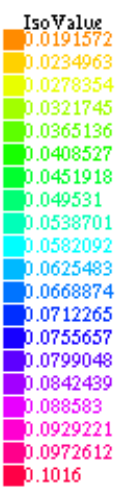

1016

Figura C.13: Estimador residual obtido na terceira iteração do refinamento adaptativo - Fig (3.7c) 
Estimador Residual
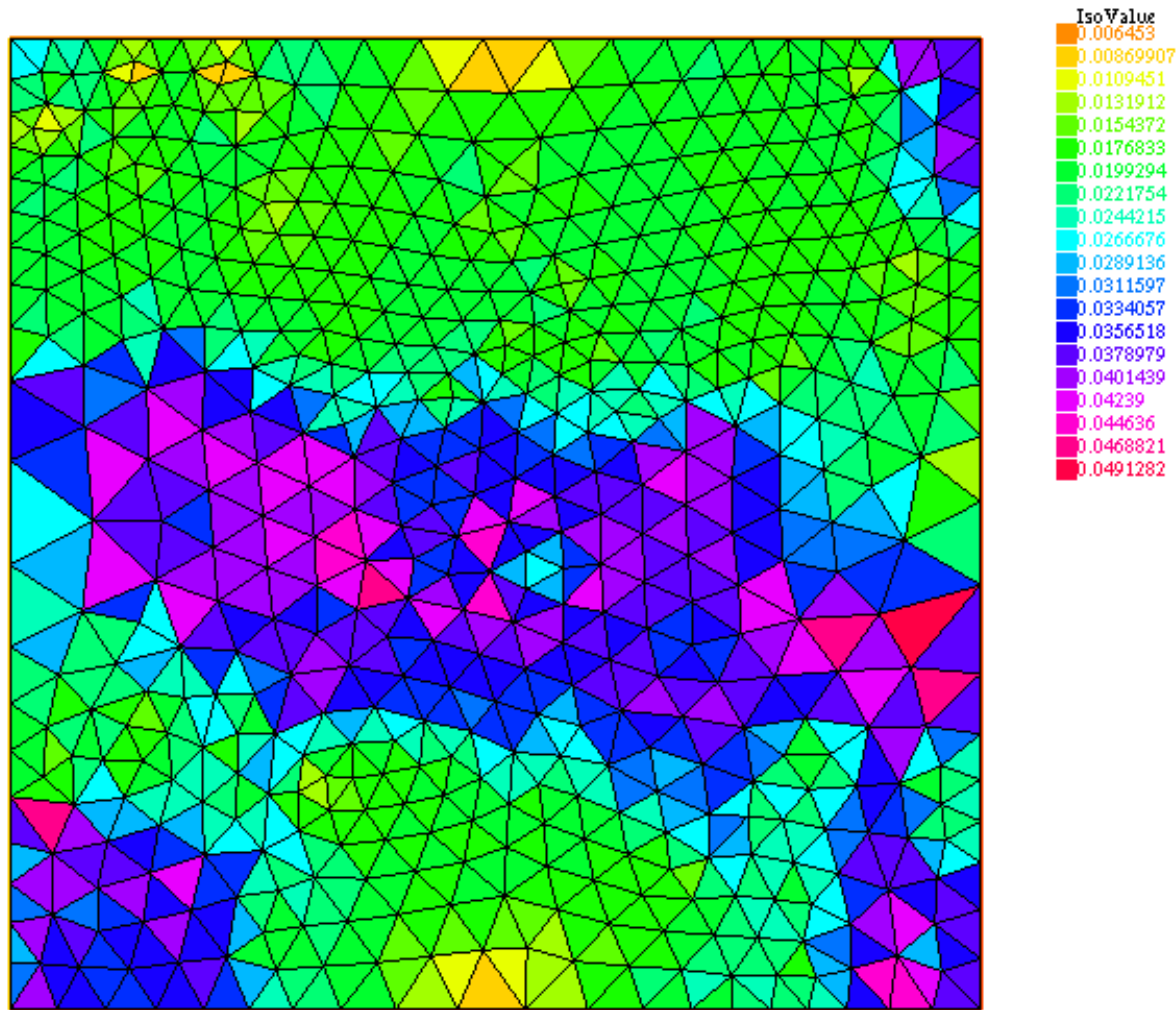

Figura C.14: Estimador residual obtido na quarta iteração do refinamento adaptativo - Fig (3.7d) 


\section{C.1.2 Problema 3.17}

Aproximacao obtida
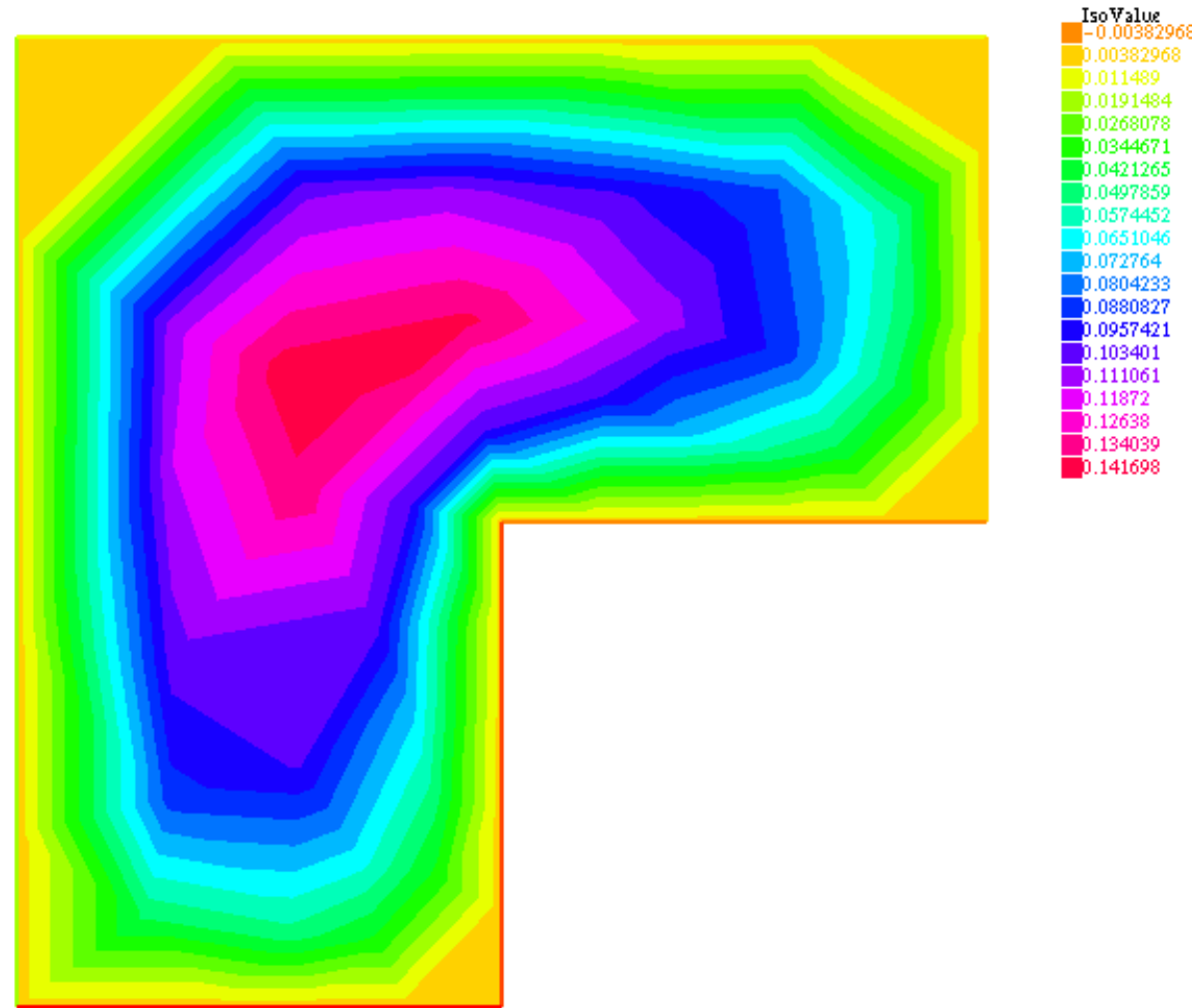

Figura C.15: Aproximação inicial para o problema 3.17 - Fig (3.9a)
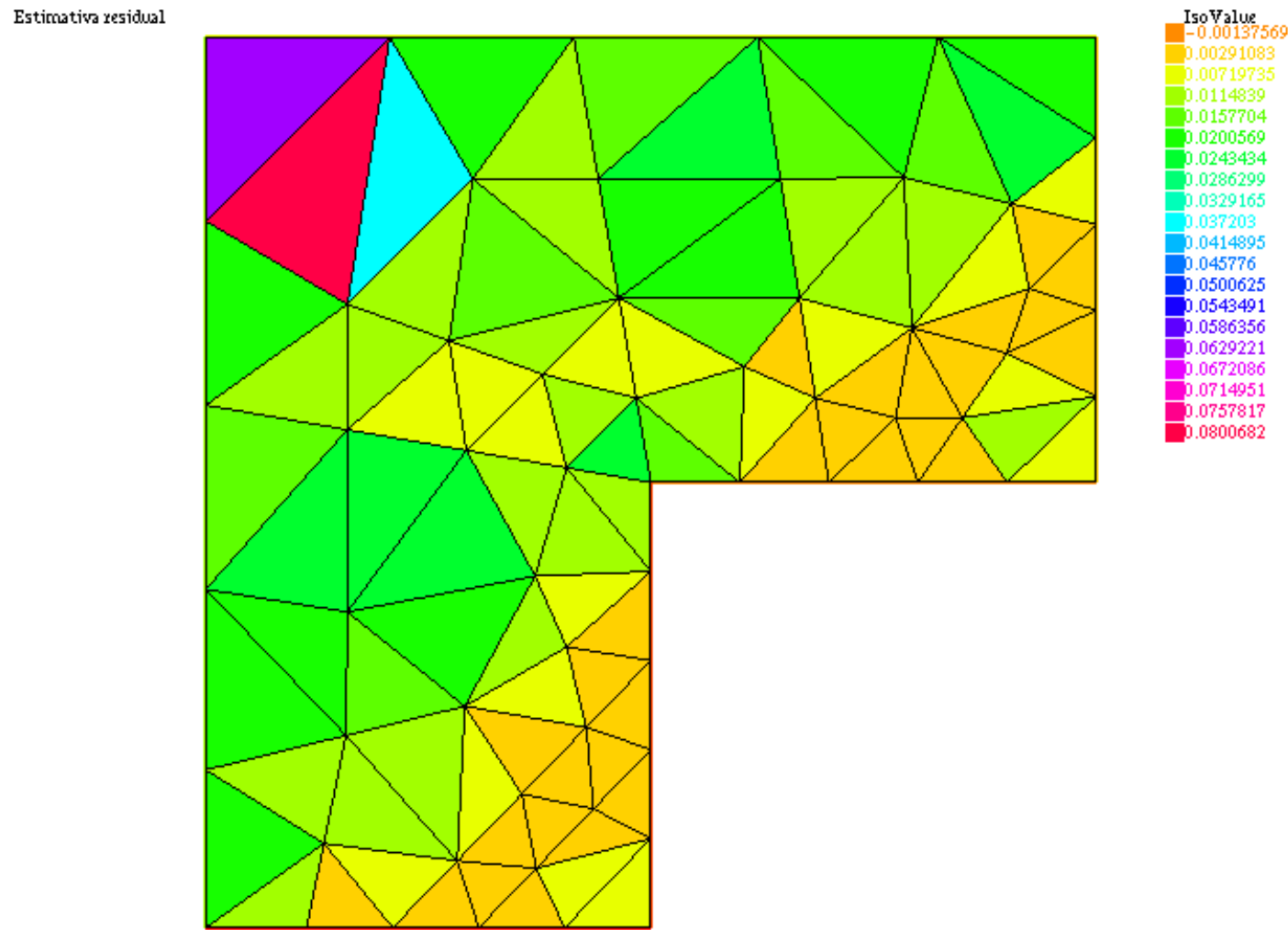

Figura C.16: Estimador residual obtido para a aproximação inicial - Fig (3.9b) 
Aproximacao obtida

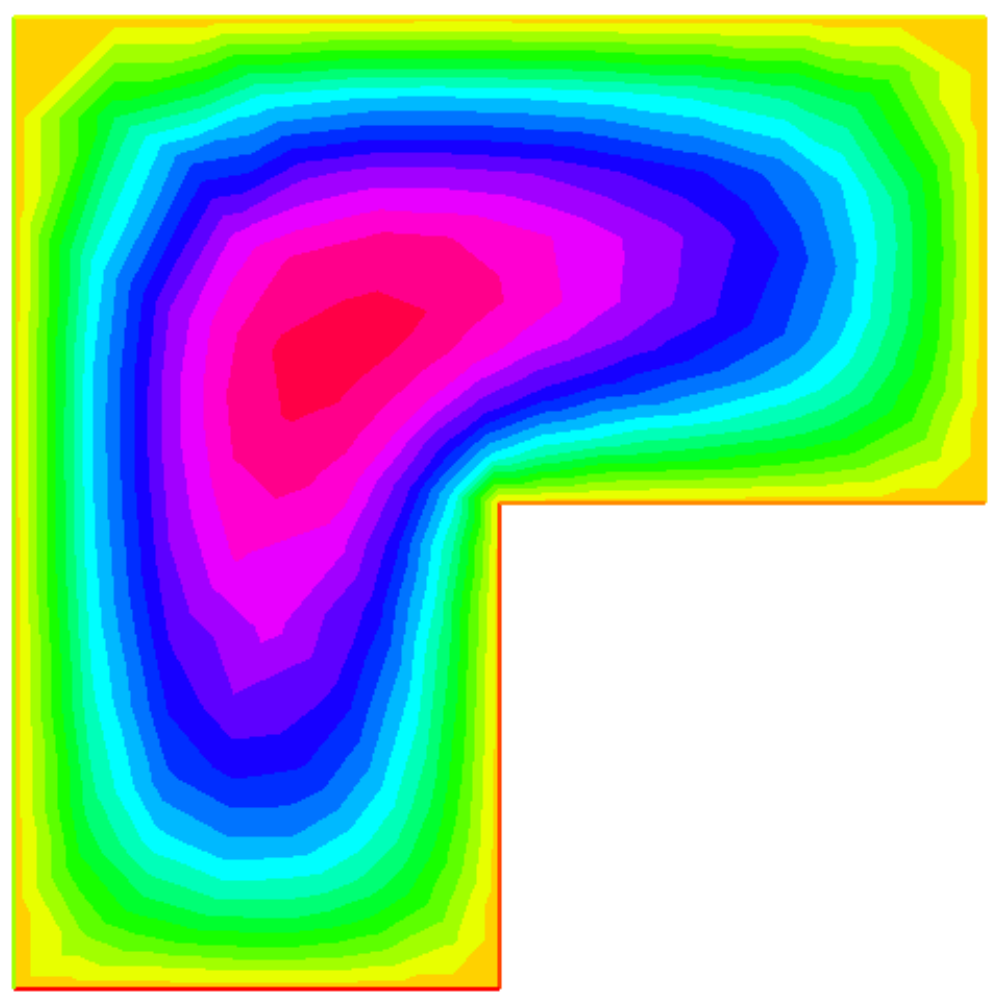

.11755

125664

0.133772
0.141879

.149986

Figura C.17: Aproximação obtida na primeira iteração do refinamento uniforme - Fig (3.10a)

Estimativa residual

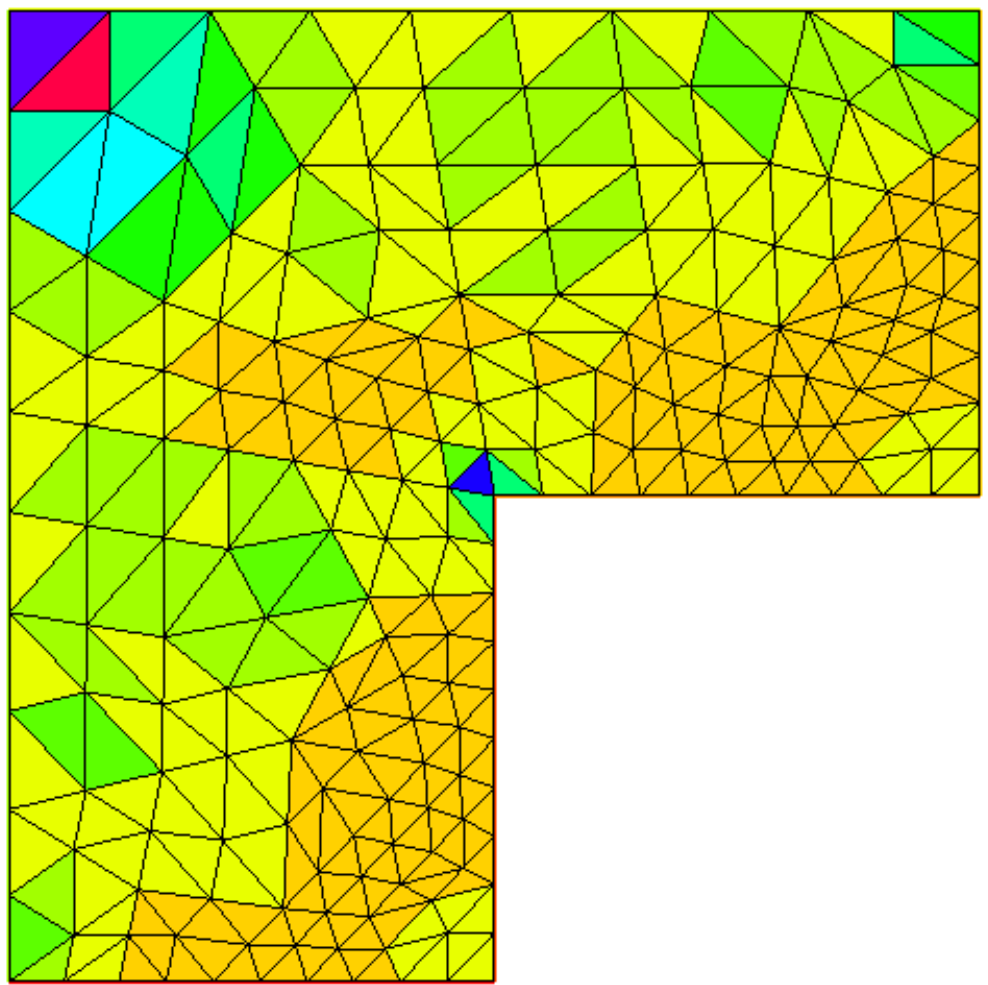

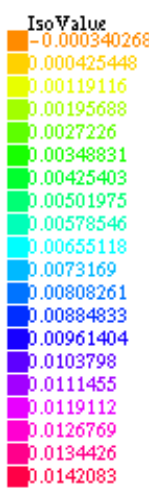

Figura C.18: Estimador residual obtido na primeira iteração do refinamento uniforme - Fig (3.10b) 
Aproximacao Obtida
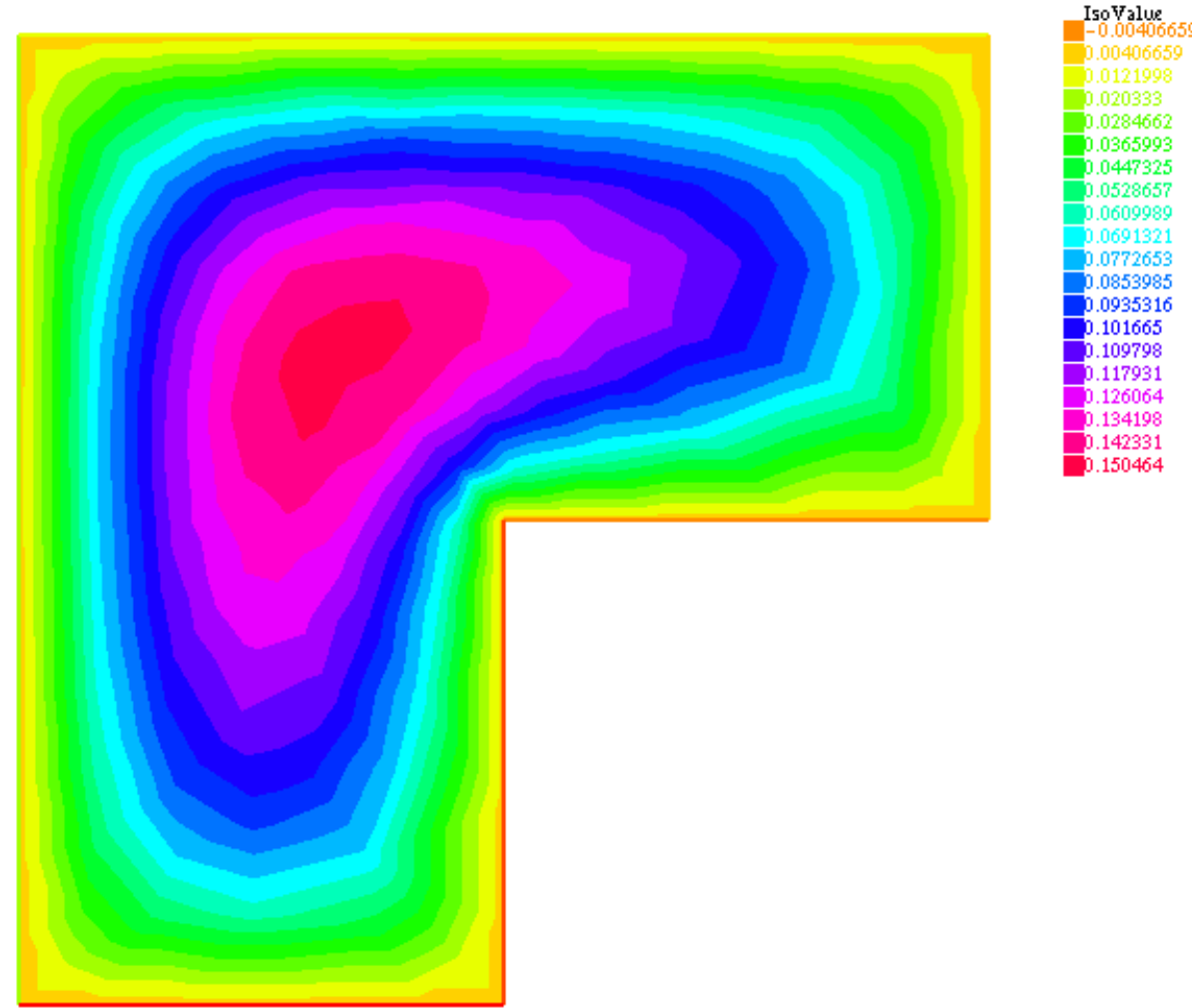

Figura C.19: Aproximação obtida na primeira iteração do refinamento adaptativo - Fig (3.11a)

Estimador Residual

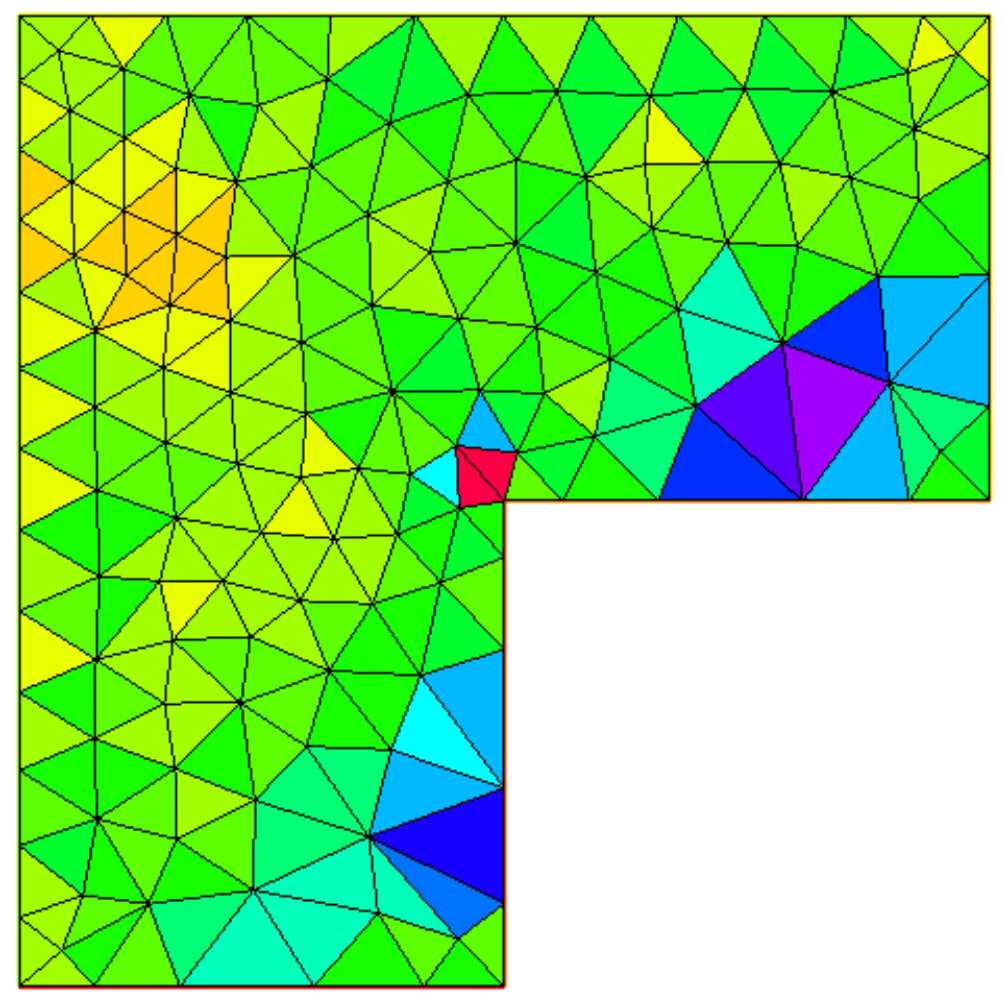

Isovalue

0.0131065

0.0275086

0.0322093

0.0418506

0.0466313

0.051412

0561927

.0657541

(1)

0800961

0848768

0.0896575

(1)

Figura C.20: Estimador residual obtido na primeira iteração do refinamento adaptativo - Fig (3.11b) 
Aproximacao obtida

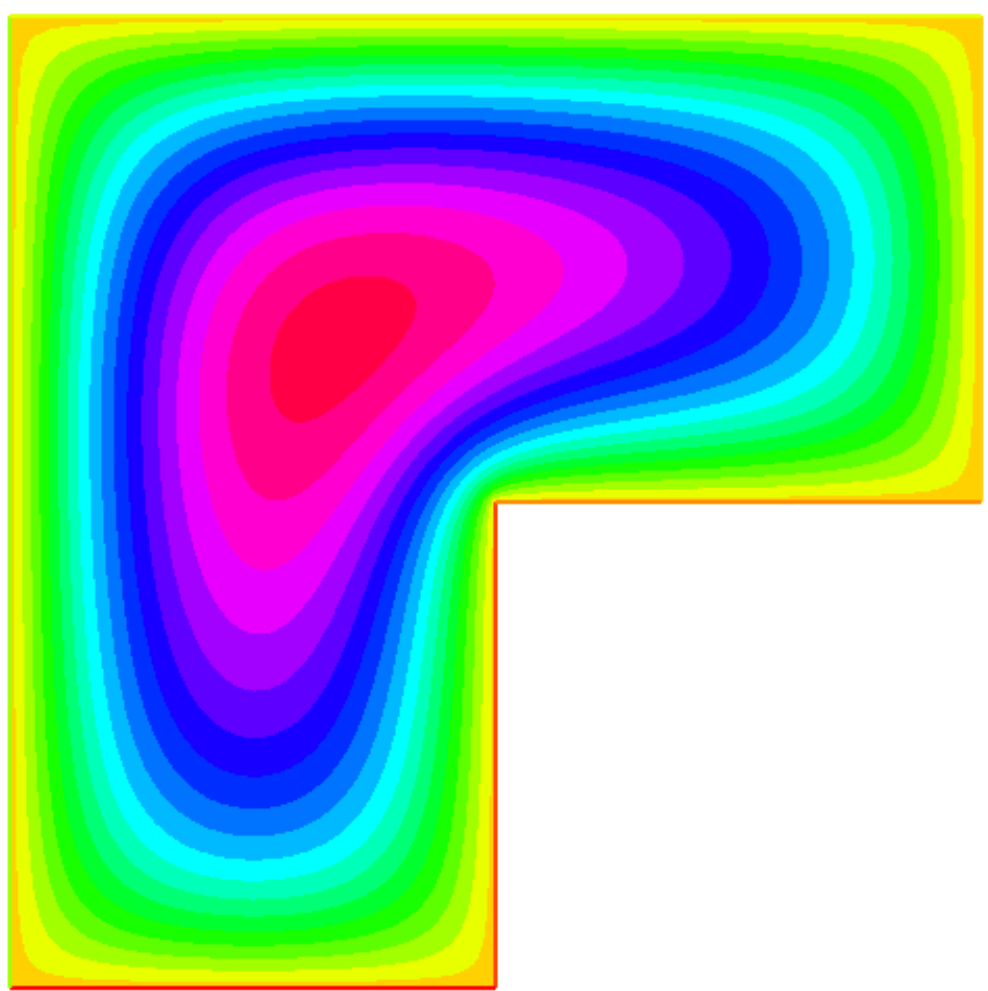

0.0373218

0.0456155

0.0539092

0.062203

0.0704967

0.0787904

0.0870841

0.0953779

0.103672

0.111965

0.12025

0.128553
0.136846

0.136846
0.14514

0.14514
0.153434

Figura C.21: Aproximação obtida na quarta iteração do refinamento uniforme - Fig (3.12a)

Aproximacao Obtida

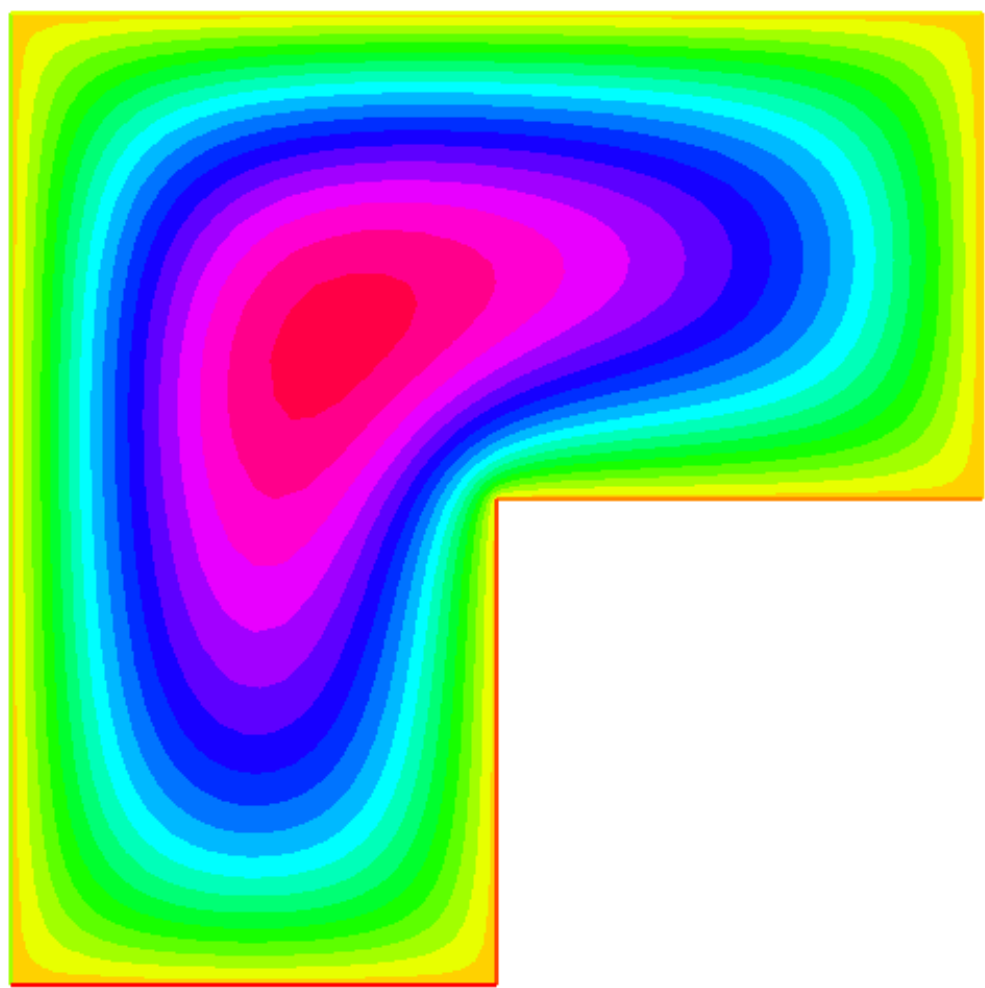

Iso Value

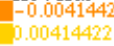

0.0124327

0.0290095

0.037298

0.0455864

0.0538748

0.0621633

0.0787401

0.0870286

.095317

.103605

0.111894

.

0.128471

$-145048$

0.153336

Figura C.22: Estimador residual obtido na quarta iteração do refinamento adaptativo - Fig (3.12b) 


\section{C.2 Elasticidade Linear}

Deslocamentos nodais
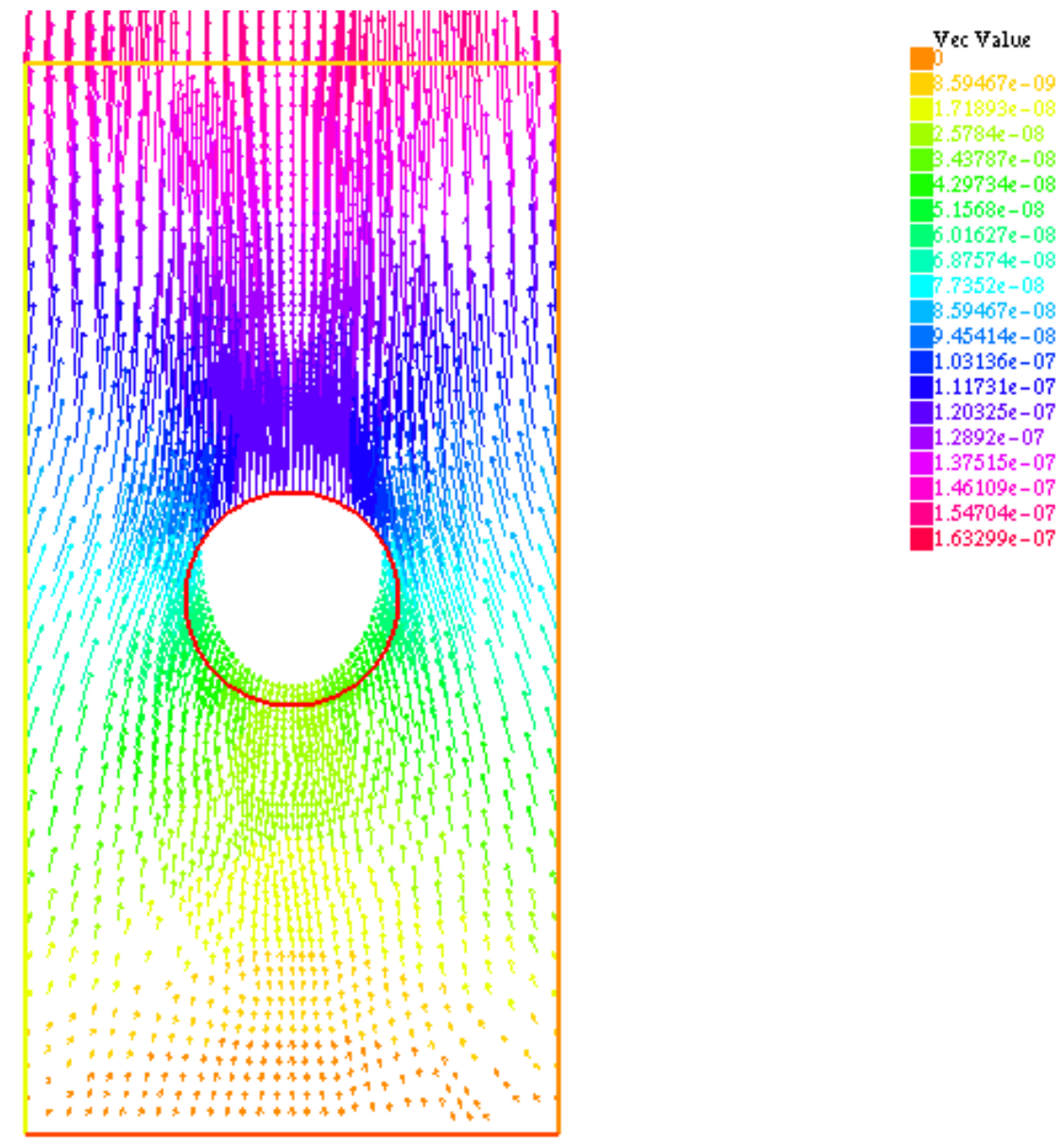

Figura C.23: Aproximação inicial para o problema da placa com furo - Fig (4.2a) 


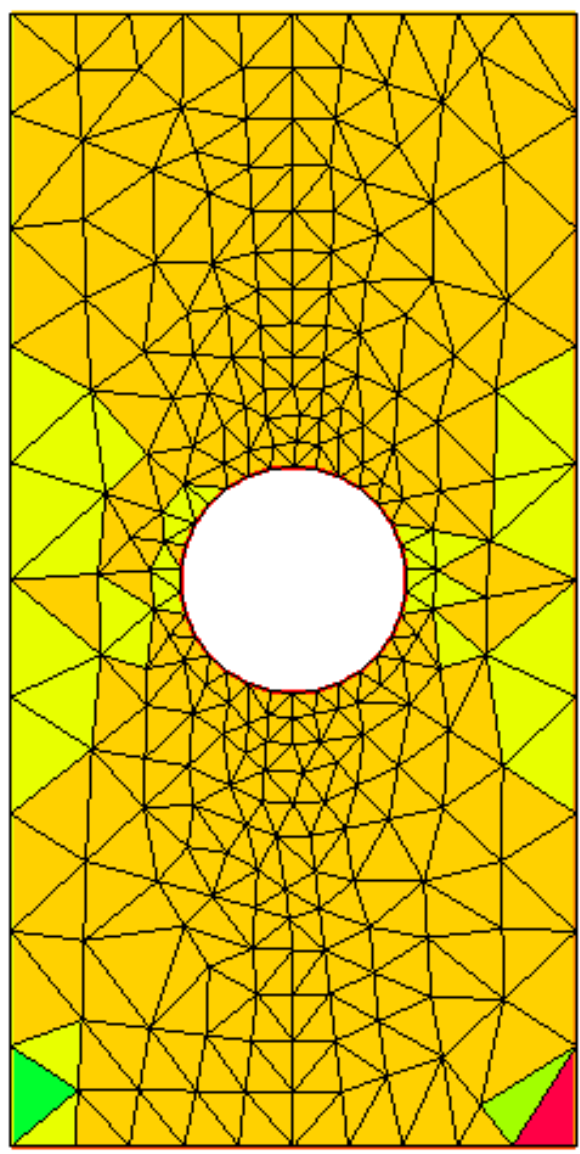

(

10108

419005
447901

76798

505694

534591

Figura C.24: Estimador residual obtido na aproximação inicial - Fig (4.2b) 
Deslocamentos nodais

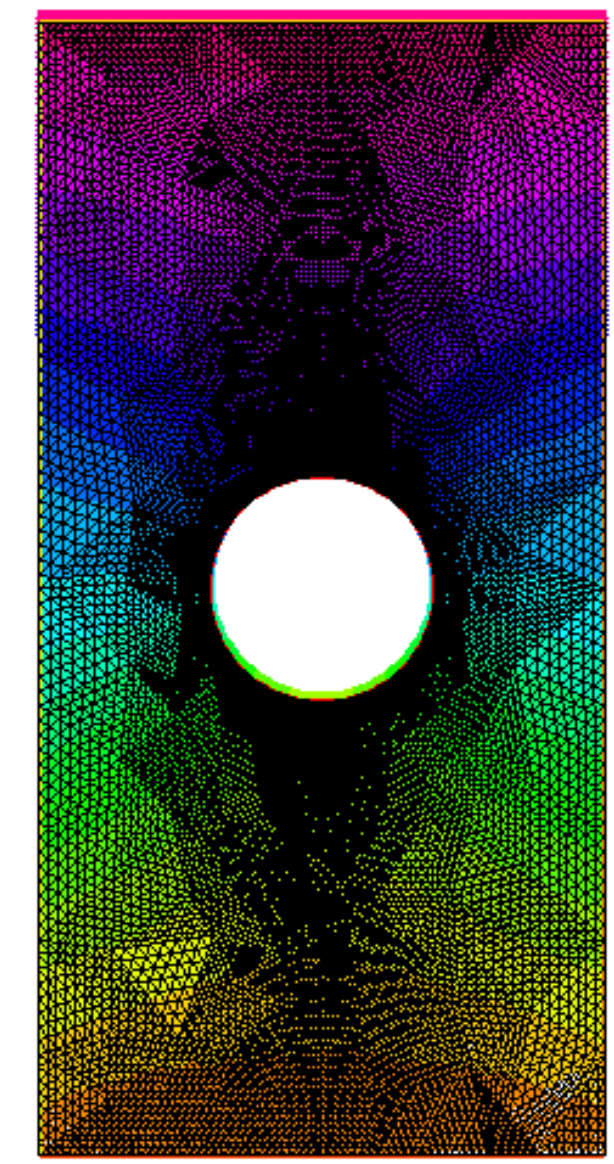

Vec Value

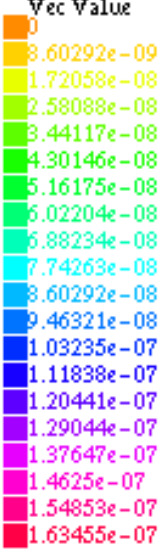

Figura C.25: Deslocamento obtido na terceira iteração de refinamento uniforme - Fig (4.4a) 
Estimativa residual

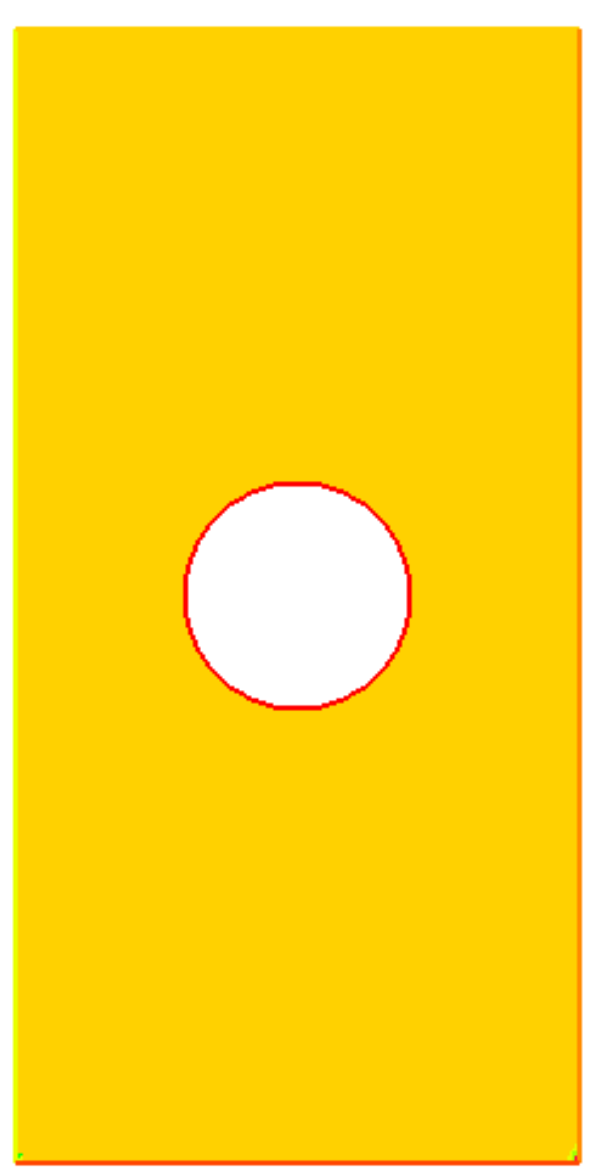

24406.9

26359.5

28312

30264.6

Figura C.26: Estimador residual obtido na terceira iteração de refinamento uniforme - Fig (4.6a) 
Estimativa residual

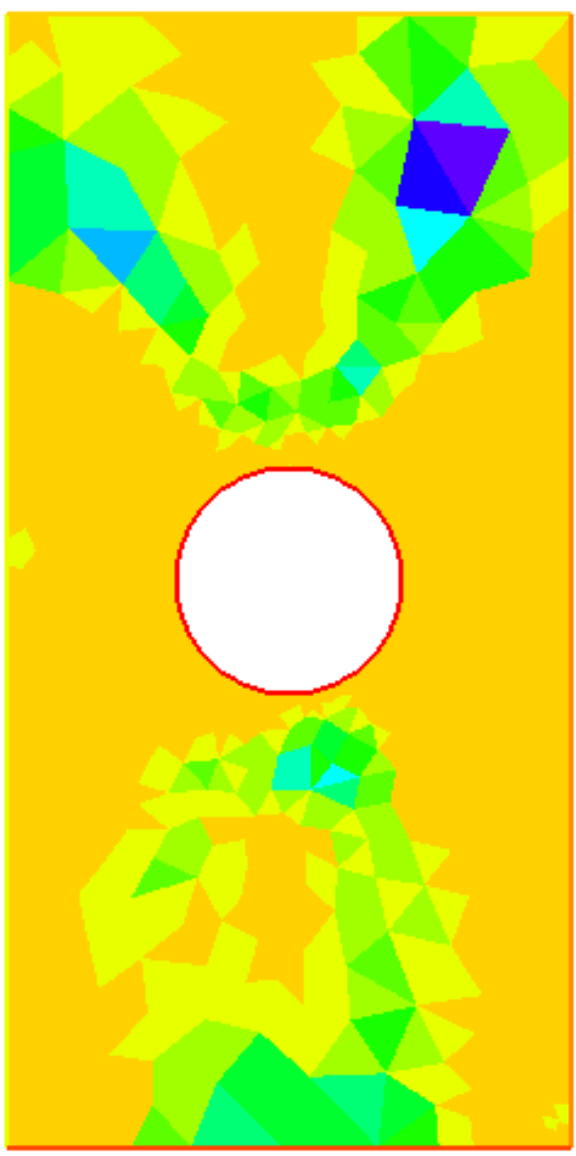

Isovalue

$-28.008$

28.0701

34.1487
-140.227
-196.306

$-196.306$

$-252.384$

- 308.463

364.542

420.62

476.699

532.777

- 588.856

644.934

701.013

757.092

813.17

$-869.249$

925.327

981.406

1037.48

Figura C.27: Estimador residual obtido na terceira iteração de refinamento adaptativo - Fig (4.6b) 
APÊNDICE C 


\section{Referências Bibliográficas}

[1] J. O. L. Demkowicz and T. Strouboulis, "Adaptive finite elements for flow problems with moving boundaries. part 1: Variational principles and a posteriori error estimates," Comput. Methods Appl. Mech. Engrg., vol. 46, pp. 217-251, 1984. 1, 2, 47

[2] J. O. L. Demkowicz and T. Strouboulis, An adaptive p-version finite element method for transient flow problems with moving boundaries. John Wiley, 1985. 1, 2, 47

[3] R. Bank, "Analysis of a local a posteriori error estimat for elliptic equations.," In Accuracy Estimates and Adaptive Refinements in Finite Element Computations, vol. 32, pp. 119-128, 1984. $1,2,47$

[4] R. Bank and A. Weiser, "Some a posteriori error estimators for elliptic partial differential equations," Math. Comp, vol. 44, pp. 283-301, 1985. 1, 2, 47

[5] I. Babutka and W. Rheinboldt, "A posteriori error estimates for the finite element method," Internat. J. Numer. Methods Engry, vol. 12, pp. 1597-1615, 1978. 1

[6] I. Babutka and W. Rheinboldt, "Error estimates for adaptive finite element computations," SIAM - J. Numer. Analysis, vol. 18, pp. 736-754, 1978. 2

[7] I. Babutka and W. Rheinboldt, "A posteriori error analysis of finite element solutions for one dimensional problems," SIAM - J. Numer. Analysis, vol. 18, pp. 565-589, 1981. 2

[8] P. Ladevèze and D. Leguillon, "Error estimate procedure in the finite element method and applications," SIAM - J. Numer. Analysis, vol. 20, pp. 485-509, 1983. 2

[9] D. Kelly., "The self-equilibration of residuals and complementary a posteriori error estimates in the finite element method," Internat. J.Numer. Methods Engrig., vol. 20, pp. 1491-1506, 1984 .

[10] B. A. Szabo, "Estimation and control of error based on p-convergence.," In Accuracy Estimates and Adaptive Refinements in Finite Element Computations, vol. 32, pp. 61-70. 2

[11] M. Ainsworth and J. Oden, A posteriori error estimation in Finite Element Analysis. Wiley, 1st ed., 2000. 2

[12] T. Grätsch and K.-J. Bathe, "A posteriori error estimation techniques in practical finite element analysis," Computer \&3 Structures, vol. 83, pp. 235-265, 2005. 2

[13] R. Verfuth, A Posteriori Error Estimation Techniques for Finite Element Methods. Oxford University Press, 2013. 2

[14] R. Verfuth, "A review of a posteriori error estimation techniques for elasticity problems," Computer Methods in applied mechanics and engineering, vol. 176, pp. 419-440, 1999. 2, 39

[15] S. C. Brenner and L. Scott, Mathematical Theory of Finite Element Methods. Springer, 3rd ed., 2008. $2,5,6,7,8,39,41$ 
[16] D. Braess, Finite Elements - Theory, fast solvers and applications in solid mechanics. Cambridge, 3rd ed., 2007. 2, 5, 7, 10, 14, 39, 41

[17] E. S. Watchen, Finite Elements and Fast Iterative Solvers with aplications in incompressible fluid dynamics. Oxford University Press, 2006. 2

[18] F. Hecht, FreeFem ++, Version 3.32. 3rd ed., 2014. 2, 5, 18, 19, 21

[19] K. Atkinson and W. Han, Theoretical Numeric Analysis: A Functional Analysis Framework. Springer, 2nd ed., 2008. 5, 7, 8

[20] L. C. Evans, Partial Differential Equations. American Mathematical Society, 1998. 7

[21] P. Ciarlet, The Finite Element Method for Elliptic Problems. Oxford, 1978. 10

[22] J. E. Flaherty, "Course notes - finite element analysis," http://www.cs.rpi.edu/ flaherje/. ix, 16

[23] G. Strang and G. J. Fix, An Analysis of the Finite Element Method. Prentice-Hall, 1973. 35 